The University of Southern Mississippi

The Aquila Digital Community

Master's Theses

Fall 12-1-2018

\title{
Late Quaternary Evolution and Stratigraphic Framework Influence on Coastal Systems along the North-Central Gulf of Mexico, USA
}

Robert Hollis

University of Southern Mississippi

Follow this and additional works at: https://aquila.usm.edu/masters_theses

Part of the Geology Commons, Geomorphology Commons, Geophysics and Seismology Commons, Oceanography Commons, Paleontology Commons, Sedimentology Commons, and the Stratigraphy Commons

\section{Recommended Citation}

Hollis, Robert, "Late Quaternary Evolution and Stratigraphic Framework Influence on Coastal Systems along the North-Central Gulf of Mexico, USA" (2018). Master's Theses. 598.

https://aquila.usm.edu/masters_theses/598

This Masters Thesis is brought to you for free and open access by The Aquila Digital Community. It has been accepted for inclusion in Master's Theses by an authorized administrator of The Aquila Digital Community. For more information, please contact Joshua.Cromwell@usm.edu. 


\title{
Late Quaternary Evolution and Stratigraphic Framework Influence on Coastal Systems along the North-Central Gulf of Mexico, USA
}

\author{
by
}

\section{Robert Hollis}

\author{
A Thesis \\ Submitted to the Graduate School, \\ the College of Arts and Sciences \\ and the School of Ocean Science and Engineering \\ at The University of Southern Mississippi \\ in Partial Fulfillment of the Requirements \\ for the Degree of Master of Science
}

Approved by:

Dr. Davin Wallace, Committee Chair

Dr. Michael Miner

Dr. Jessica Pilarczyk

Dr. Davin Wallace

Committee Chair
Dr. Jerry Wiggert

Associate Director
Dr. Karen S. Coats

Dean of the Graduate School

December 2018 


\title{
COPYRIGHT BY
}

\author{
Robert Hollis
}

2018

Published by the Graduate School

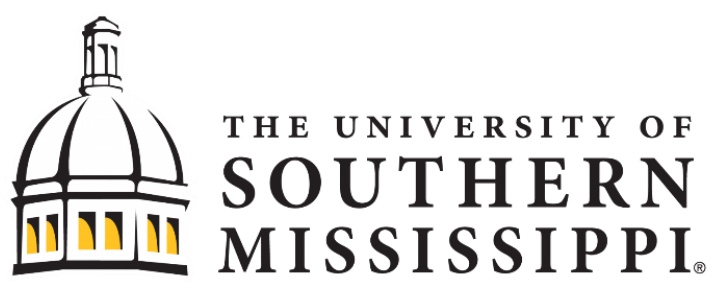




\begin{abstract}
Coastal systems in the Gulf of Mexico are threatened to reduced sediment supply, storm impacts and relative sea level rise (RSLR). The geologic record can provide insights of geomorphic threshold crossings (formation, progradation, transgression, destruction) to these forcing mechanisms to predict future barrier evolution to climate change. The stratigraphic framework and antecedent topography directly influence coastal evolution over geologic timescales. This study synthesizes $\sim 2100 \mathrm{~km}$ of geophysical data, 700+ sediment cores, and 63 radiocarbon dates to regionally map two sequence boundaries, multiple ravinement surfaces and fourteen depositional facies. One marine isotope stage (MIS) 6 valley's fill provided up to $300 \times 10^{6} \mathrm{~m}^{3}$ of sand to modern systems through transgressive ravinement during the Holocene. Repeated storm breaches or tidal inlets correspond to paleotopographic low's in the MIS 2 surface.

A Holocene geomorphic evolutionary model was created for Petit Bois and Dauphin Island from available data, highlighting RSLR rates and sediment supply. As the MIS 2 surface was flooded, tidal/wave scour supplied sand to migrating marine shoals. These rapidly transgressing shoals converted drowned paleovalleys to estuaries starting about 9ka. Islands formed in their modern positions about 6ka, when sediment supply was high and RSLR rates were $2 \mathrm{~mm} / \mathrm{yr}$. Between $4 \mathrm{ka}-1750 \mathrm{CE}$. Islands prograded due to RSLR rates of $1-0.4 \mathrm{~mm} / \mathrm{yr}$ and sufficient sand supply from alongshore and inner shelf sources. Currently, the islands experience RSLR rates of $3.61 \mathrm{~mm} / \mathrm{yr}$ and reduced sediment supply resulting in barrier degradation. This study provides geologic evidence of coastal geomorphic thresholds related to RSLR, sediment supply and antecedent topography.
\end{abstract}




\section{ACKNOWLEDGMENTS}

I would like to thank my advisor, Dr. Davin Wallace for his guidance and great support through this project. I would also like to thank my committee members Dr. Michael Miner and Dr. Jessica Pilarczyk, and lab mates, Clayton Dike, Nina Gal, and Shara Gremillion for their helpful and insightful feedback and guidance.

This project was funded by the Bureau of Ocean and Energy Management, as part of a Gulf of Mexico sand resource mapping effort. Thank you to colleagues at the University of Alabama, the Geological Survey of Alabama and USGS for allowing core sampling. The USGS and UTIG shared archive geophysical data. Mollusk bivalve identification was greatly aided by Dr. Jennifer Walker. Dr. John Anderson (Rice) and Dr. Ervin Otvos (USM) also provided valuable insight. 


\section{DEDICATION}

This thesis is dedicated to friends, family and loved ones especially Bob, Ellen, Joey and Alli. Their love, appreciation and support allowed me to pursue my scientific passion. 


\section{TABLE OF CONTENTS}

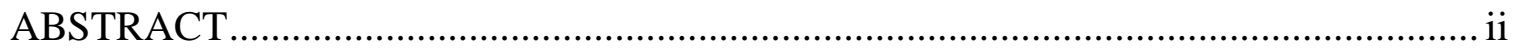

ACKNOWLEDGMENTS .............................................................................. ii

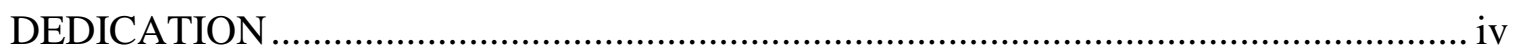

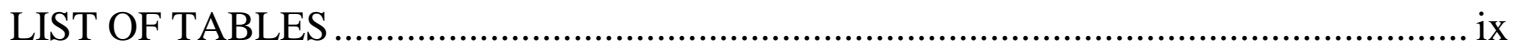

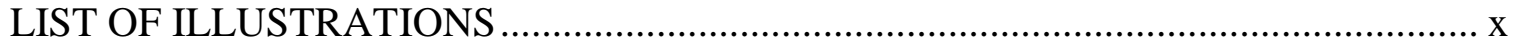

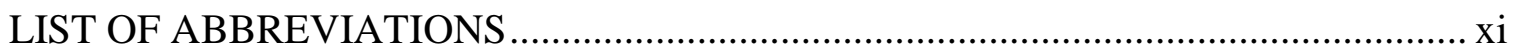

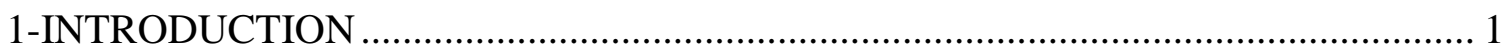

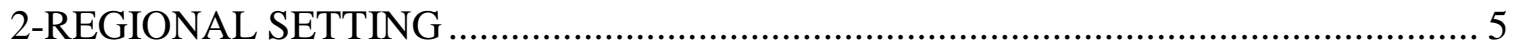

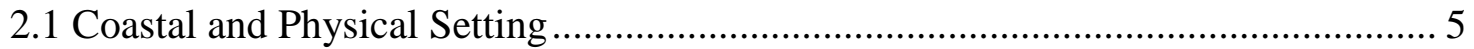

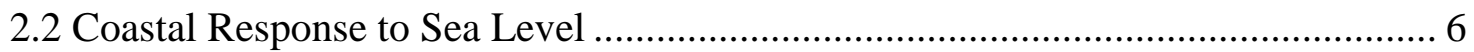

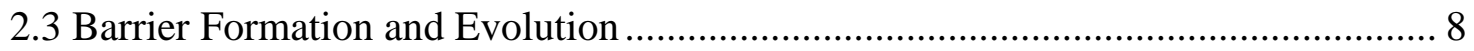

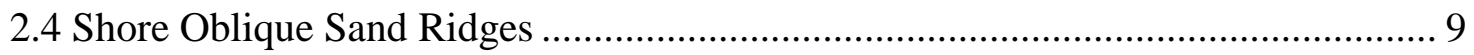

2.5 Mississippi/Alabama Coastal Geology .......................................................... 10

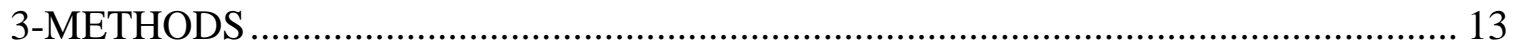

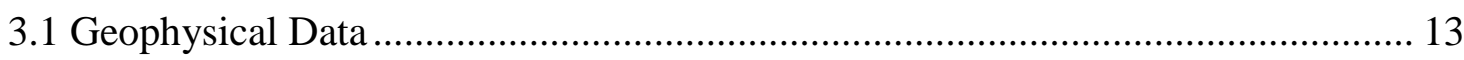

3.2 Sediment Cores .......................................................................................... 15

3.3 Radiocarbon Dating Estimates.................................................................... 15

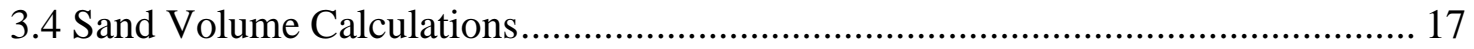

3.5 Evolutionary Model ................................................................................. 18 
4.1 Quaternary Chronostragraphic Framework ....................................................... 19

4.1.1 Sequence Boundary 1: Marine Isotope Stage 6 ....................................... 25

4.1.2 Sequence Boundary 2: Marine Isotope Stage 2 ........................................ 27

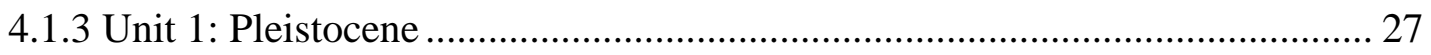

4.1.4 Unit 2: Fluvial Channel Sands Deposits ............................................... 28

4.1.5 Unit 3: Fluvial Lateral Accretion Deposits ................................................. 28

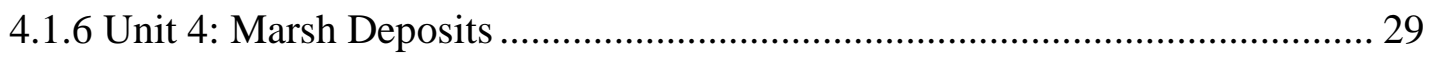

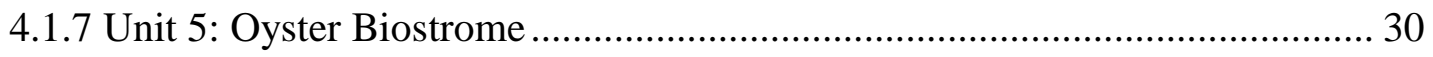

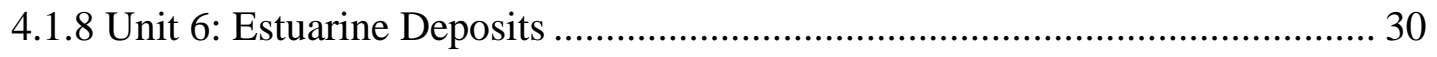

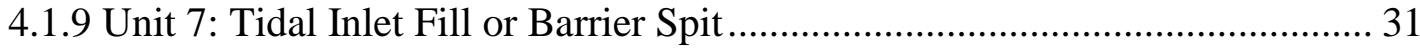

4.1.10 Unit 8: Flood Tidal Delta Deposits ....................................................... 31

4.1.11 Unit 9: Ebb Tidal Delta Deposits (Modern) …......................................... 32

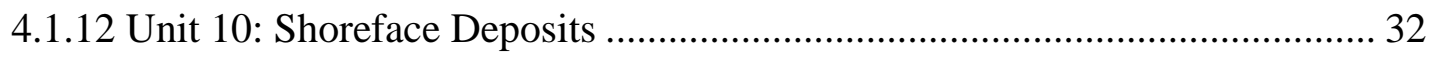

4.1.13 Unit 11: Barrier and Backbarrier Deposits ................................................ 33

4.1.14 Unit 12: Marine Surficial (MAFLA) Sand Sheet ....................................... 34

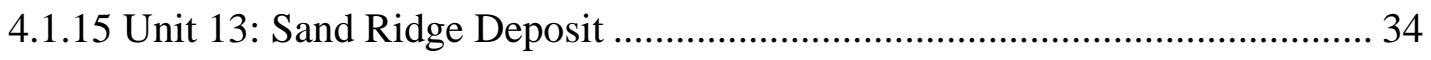

4.1.16 Unit 14: Inner Shelf Mud Deposit .................................................... 35

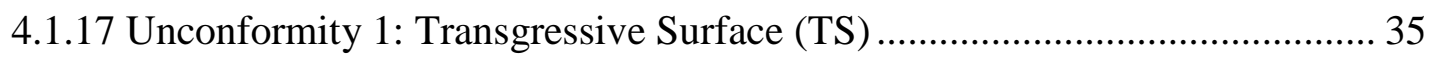

4.1.18 Unconformity 2: Wave Ravinement Surface (wRs) ................................ 35 


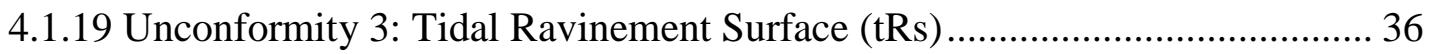

4.1.20 Unconformity 4: Bay Ravinement Surface (bRs) ..................................... 36

4.1.21 Unconformity 5: Sand Ridge Ravinement Surface (sRs) ............................ 38

4.2 Antecedent Topography and Paleofluvial systems ......................................... 38

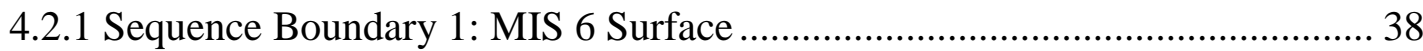

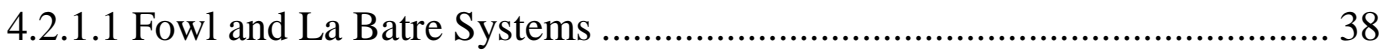

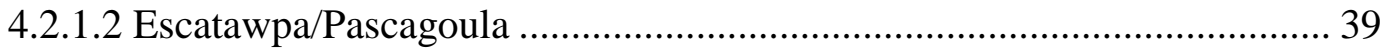

4.2.2 Sequence Boundary 2: MIS 2 Surface ................................................... 40

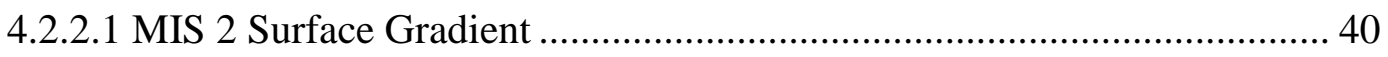

4.2.2.2 Fowl, La Batre, Escatawpa Systems .................................................. 41

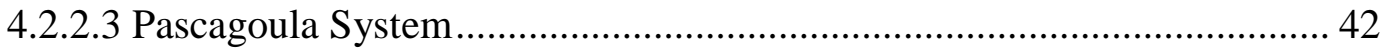

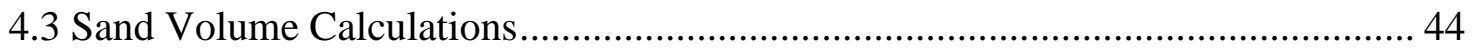

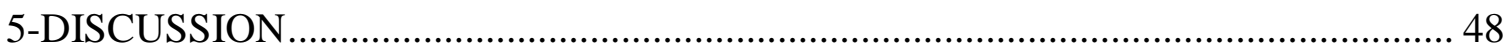

5.1 Pre-Holocene Antecedent Geology and Topography Influences........................ 48

5.2 Sediment Sourced through Transgressive Ravinement .................................... 50

5.3 Holocene Transgressive Evolutionary Model................................................ 51

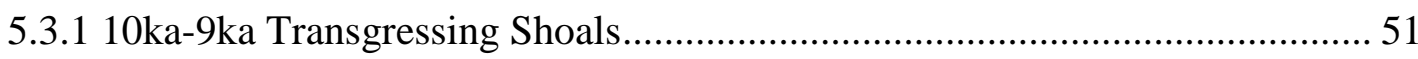

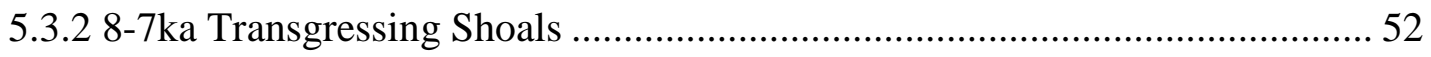

5.3.3 6-5ka: Island Formation and Stabilization .............................................. 55

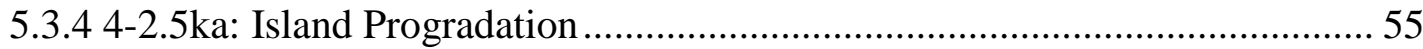


5.3.5 1750 CE- Modern: Island Degradation.

6-CONCLUSION

REFERENCES 


\section{LIST OF TABLES}

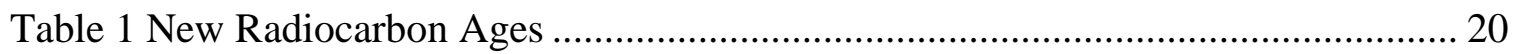

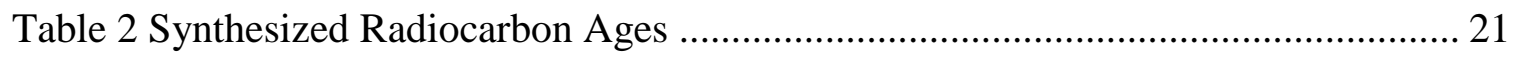

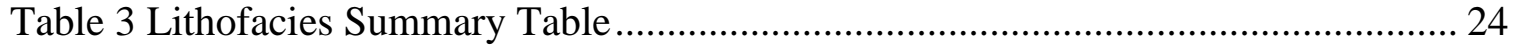

Table 4 Sand Source and Sink Volumes............................................................... 45 


\section{LIST OF ILLUSTRATIONS}

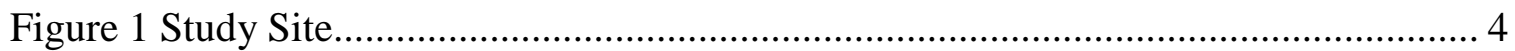

Figure 2 Eustatic and Texas/Louisiana Sea Level Curves .......................................... 8

Figure 3 MSAL Coastal Geology and Lidar......................................................... 11

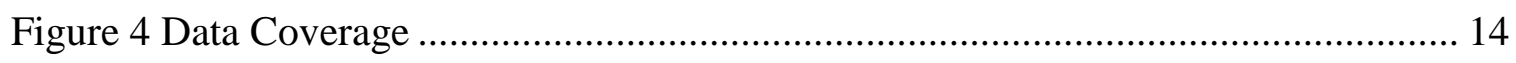

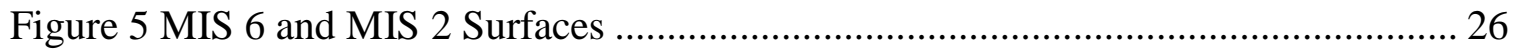

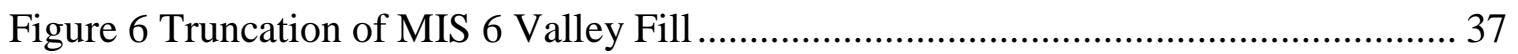

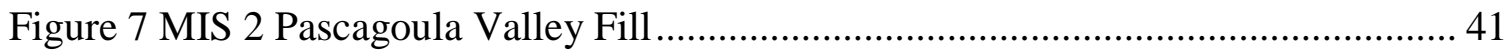

Figure 8 Petit Bois and Dauphin Shoreface ................................................................ 43

Figure 9 Petit Bois Shore Parallel Stratigraphic Cross Sections................................... 46

Figure 10 Dauphin Shore Parallel Stratigraphic Cross Sections ................................... 47

Figure 11 Holocene Evolutionary Model ................................................................ 54 


\section{LIST OF ABBREVIATIONS}

MSAL

$m b s l$

masl

$r s l r$

$r s l$

mis

$C E$

BP

$k a$

$D E M$
Mississippi-Alabama

meters below sea level

meters above sea level

relative sea level rise

relative sea level

marine isotope stage

common era

before present

kilo annum (1,000 years)

digital elevation model 


\section{1-INTRODUCTION}

Coastal environments along the Gulf of Mexico are threatened due to sea-level rise, reduced sediment supply, and storm impacts, which will only be exacerbated with climate change. Increased focus has shifted to modeling the future impacts of these climatic forcings. However, observational data used to constrain models are usually limited to the instrumental record, which do not capture longer-term barrier system geomorphic threshold responses (island formation, stabilization, progradation, transgression, destruction). These geomorphic threshold responses result from forcing mechanisms such as changes in sea level increasing accommodation space by flooding antecedent topography (Rodriguez et al. 2004, 2008, Anderson et al. 2014) and changes in sediment supply (Raff et al. 2018, Rodriguez et al. 2018, Odezulu et al. 2018). Many studies show the most complete records of coastal evolution are preserved within paleofluvial incised valleys and partially in antecedent shelf deposits (Anderson et al. 2014, Mallinson et al. 2010, Zaremba et al. 2016, Nordfjord et al. 2005). Comparing the chronologic evolution of these preserved environments to well-constrained Holocene sea level curves sheds valuable insight toward geomorphic threshold parameters (Milliken et al. 2008, Rodriguez et al. 2010). However, research concerning how the regional stratigraphic framework influences modern coastal geomorphology and sediment supply variability is limited to a few study areas (Riggs et al. 1995, Zaremba et al. 2016, Mallinson et al. 2010, Anderson et al. 2016, Belknap and Kraft 1985) and is yet to be constrained for the Mississippi-Alabama (MSAL) barrier island chain.

The MSAL shelf is comprised of a complex network of coastal plain and piedmont incised valley systems (Greene et al. 2007) with compound and simple fill 
structures (Dalrymple et al. 1994, Zaitlin et al. 1994). Incised valley geometry and fill architecture controls are related to base-level fall rates and magnitude, shelf gradient, drainage basin geology, subsidence, sediment discharge and climate (Anderson et al. 2004, Mattheus et al. 2007 and references within). This is the major mechanism creating topography in otherwise low gradient coastal settings. Previous studies on the MSAL shelf focus on the five shelf edge deltas (Kindinger 1989, Roberts et al. 2004, Fillon et al. 2004, Bartek et al. 2004) and shelf valleys (Bartek et al. 2004, Flocks 2015, Kindinger 1988, Kindinger et al.1994). However, there is disagreement concerning the timing and location of large depositional features and associated feeder valleys (Roberts et al. 2004, Bartek et al. 2004, Greene et al. 2007). For example, Gonzalez et al. (2017) reported in situ cypress stumps and peats of pre-MIS 2 age offshore of Fort Morgan, Alabama in $\sim 20 \mathrm{~m}$ water depths. Based on elevation estimates and sea level data the valley fill was estimated to be Holocene (Bartek et al. 2004). Entire sea level cycles may not be preserved, increasing the complexity of the sequence stratigraphy. Furthermore, these studies do not incorporate how these systems influenced Holocene barrier island morphodynamics and generally lack upland fluvial source mapping. The most complete stratigraphic records of coastal evolution are preserved within fluvial incised valleys in highly dynamic settings (Anderson et al. 2014, Mallinson et al. 2010, Zaremba et al. 2016).

Shorelines of the Gulf of Mexico are among the most vulnerable in the United States (Pendleton et al., 2010), and policy and management decisions should incorporate coastal geology (Dolan and Wallace, 2012). They demonstrate highly variable geologic frameworks, sediment flux, subsidence rates and types of evolution, even across 
relatively small spatial scales (Anderson et al. 2004, Otvos 2018). The geologic framework and inherited antecedent topography are critical factors impacting island progradational, retrogradational and or aggradational evolution modes (Raff et al. 2018, Timmons et al. 2010), paleovalleys anchoring tidal inlets (Mallinson et al. 2010) and erosional hotspots (Twichell et al. 2013, Hapke et al 2016, Honeycutt and Krantz 2003, Browder and McNich 2006). Stratigraphic framework, antecedent topography and relative sea level rise rates also strongly influences wave ravinement depths, which vary greatly across Texas (8-12m) (Rodriguez et al. 2001, Wallace et al. 2010; Wallace and Anderson, 2013) and Louisiana (10-16m) (List et al. 1994). These same factors along with tidal prism and the number of inlets control the depth of tidal ravinement (Miner et al. 2007, 2009, FitzGerald et al. 2004, Cattaneo and Steel 2013). Together, these transgressive ravinement processes have important implications when estimating barrier sediment supply from reworked offshore relict deposits (Weight et al. 2011) or in times of rapid relative rise, their preservation (Swift et al. 1975). Relict falling stage deltas reworked during the Holocene transgression were the principle sand source for modern coastal systems in Texas (Anderson et al. 2016).

Quantifying the Late Quaternary coastal evolution in the northern Gulf of Mexico (GOM) provides a link between the inner shelf geologic framework and the modern MSAL coastal geomorphology. Another objective of this study is to constrain forcing mechanisms such as changes in relative sea level rise (RSLR) rates and sediment supply over geologic timescales in a regional context that can be applied to similar systems (Rodriguez et al. 2010, Anderson et al. 2016). Both are critical to improve our understanding of coastal geomorphic thresholds moving forward, given that current 
relative sea level rise rates have exceeded rates during island formation (Otvos 2018) and the likely future increase in storm frequency and intensity (Emanuel 2005). This study aims to holistically synthesize many previous works in the area (Flocks 2015, Flocks et al. 2014, Twichell et al. 2011, McBride et al. 1991, Kramer 1990, Greene et al. 2007, Hummel and Parker 1995, Hummel and Smith 1996, Otvos 1979, 1981, 1985, 1986, 2018) supplemented with newly acquired geophysical and chronologic data to investigate the coevolution of the barrier islands and other coastal systems, highlighting sand supply through ravinement of the inner shelf.

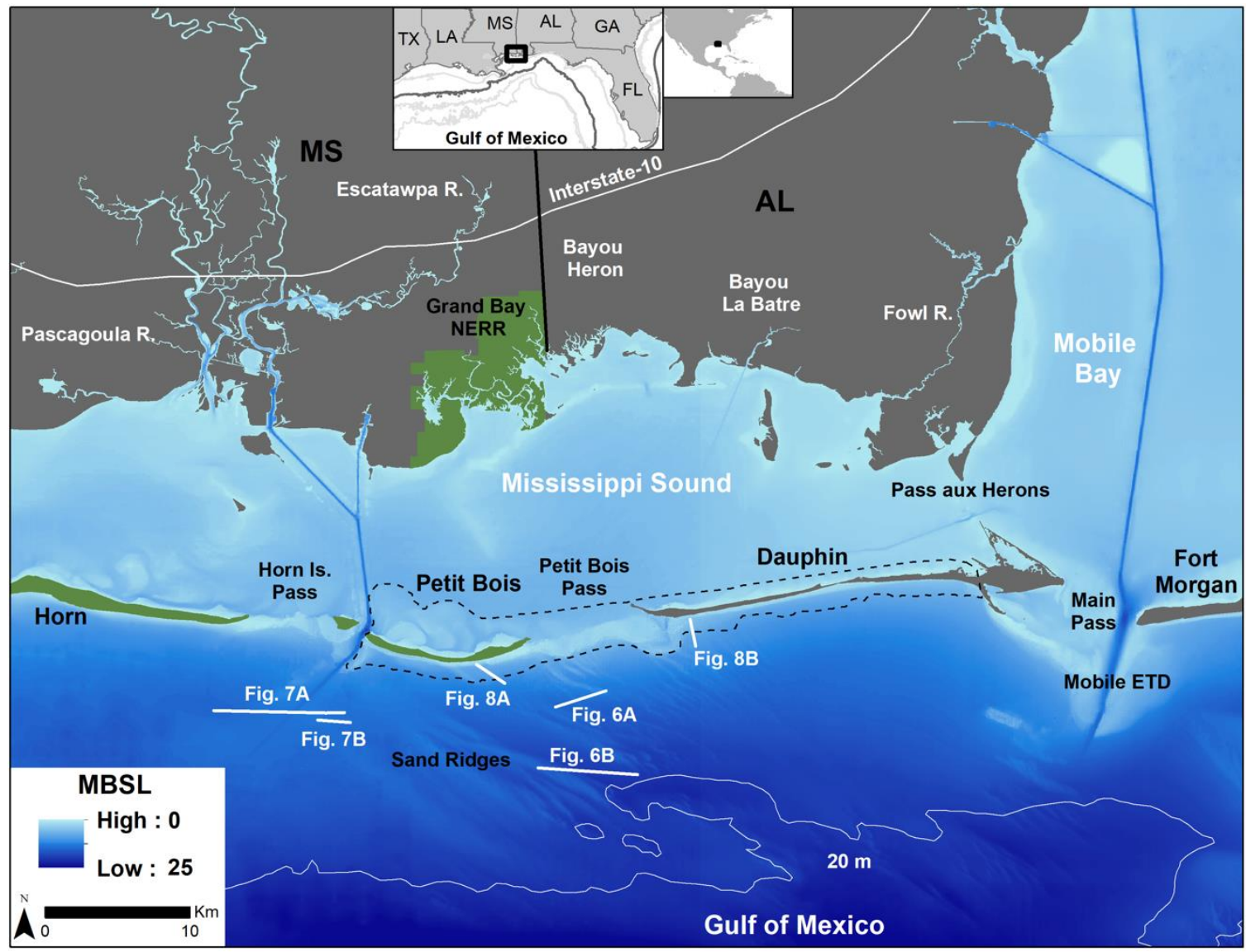

Figure 1 Study Site

Map of Petit Bois, MS and Dauphin Island, AL. Green areas represent natural preservation areas ie., Gulf Island National Seashore or Grand Bay National Estuarine Research Reserve (NERR). Figure 6, 7, 8 locations shown in white bold lines, dotted black lines represent the sand extent of the active barrier system used for sand volume calculations (table 4). 


\section{2-REGIONAL SETTING}

\subsection{Coastal and Physical Setting}

The microtidal, storm dominated, MSAL shelf is a slowly subsiding ( $2 \mathrm{~mm} / \mathrm{yr})$, low gradient, passive margin (Ivins et al. 2007, Flocks 2015). The MSAL barrier chain is located about 5-20km offshore, separating the Gulf of Mexico from the Mississippi Sound (Figure 1). This wind dominated estuary, has a $1.23 \times 10^{9} \mathrm{~m}^{3}$ large tidal prism (Eleuterius, 1980). Tidal currents are $0-0.91 \mathrm{~m} / \mathrm{s}$, but vary between inlets (Rucker and Snowden 1990). Meteorological forcings such as frontal passages strongly influence water levels and current velocities.

The significant wave height at $15 \mathrm{~m}$ water depth on the inner shelf, ranges from $0.4 \mathrm{~m}-0.7 \mathrm{~m}$ in the summer and winter respectively, which reflects seasonal differences in passing cold fronts and tropical cyclones (NDBC 2016: Buoy 42007, Eisemann et al. 2018). Onlapping marine muds at a depth of $7 \mathrm{~m}$ mark the toe of the shoreface at the western end of the MSAL barrier chain at Ship Island (Twichell et al. 2013). Shallow cores (Kelso and Flocks 2015) and geophysical data (Forde et al. 2011a, 2011b, 2013) offshore Petit Bois show the toe of the shoreface at 8-10m. Dauphin Island's shoreface toe is marked by exposed Pleistocene deposits at $7 \mathrm{~m}$. Dominant southeasterly swells create a net westward longshore current flowing at 0.5-1.5 m/s (Boone 1973). The entire MSAL chain shows sediment transport connection to the Mobile ebb tidal delta to some degree (Byrnes et al. 2013); although significant amounts of sediment are sequestered in tidal deltas and dredge-maintained shipping lanes (Cipriani and Stone 2001).

Mississippi Sound's salinity ranges from nearly fresh to $36 \mathrm{psu}$ from the multiple river systems (Upshaw et al. 1966). The piedmont Mobile-Tensaw River is the sixth 
largest river system in the contiguous United States with a drainage basin of $133,500 \mathrm{~km}^{2}$ and an average discharge of $1,788 \mathrm{~m}^{3} / \mathrm{s}$ (Greene et al. 2007). Roughly $15 \%$ of its discharge enters Mississippi Sound through Pass Aux Herons (Hummel and Parker 1995). The Pascagoula/Escatawpa River system has a drainage basin of $25,122 \mathrm{~km}^{2}$ and discharge of $271 \mathrm{~m}^{3} / \mathrm{s}$ (Newcome 1967). The Pascagoula is the largest undammed river in the contiguous United States. The coastal plain Fowl River system has a drainage basin of $197 \mathrm{~km}^{2}$ and an average discharge $1 \mathrm{~m}^{3} / \mathrm{s}$. The coastal plain, meandering La Batre River drainage basin is $75 \mathrm{~km}^{2}$ and has an average discharge of $0.4 \mathrm{~m}^{3} / \mathrm{s}$ (Greene et al. 2007).

\subsection{Coastal Response to Sea Level}

Sea level cycles are the driving mechanism for coastal evolution over geologic timescales (Anderson et al. 2016). Shackleton (1987) relates marine oxygen isotope records and global ice volume as proxies for eustatic sea level (Figure 2). Large swings in the oxygen isotope data observed from deep sea foraminifera represent global climatic changes between glacial and interglacial periods or marine isotope stages (MIS) (Emiliani 1955). Depositional environments and stratigraphic sequences are directly referenced to sea level cycles in this study similar to other work in Texas (Anderson et al. 2004). During the MIS 6 lowstand, global sea level was about 130 to 140m below modern about 155-136 ka (Figure 2) (Shackleton 1987, 2000). Other studies in Louisiana (Wellner et al. 2004), central Texas (Abdulah et al. 2004), and MSAL (Greene et al. 2007) show larger MIS 6 valley width, greater incisional depth and sediment load compared to the most recent MIS 2 lowstand. This is supported by the large MIS 6 or 8 Lagniappe Delta on the MSAL shelf (Roberts et al. 2004). 
During the MIS 5e highstand, sea level was 3-6m above present (Otvos 2005, 2018). A discontinuous barrier ridge complex spans most of Texas through Florida (Otvos 2018). MIS 2-5 represents falling stage to lowstand tracts where global sea level fell to 120 meters below modern about 18,000-26,000 years ago (Shackleton 1987). The MSAL shelf consists of a series of lowstand (MIS 2 and older) incised paleofluvial valleys and fluvial channel deposits (Bartek et al. 2004, Flocks 2015, Greene et al. 2007). Previous studies suggest the MIS 2 surface dips to the southwest (Otvos 1985). These incised valleys were filled with fluvial to marine transgressive sediments during the Holocene and Late Pleistocene (Figure 3), preserving the most complete stratigraphic record on the shelf (Bartek et al. 2004, Flocks et al. 2015).

Detailed composite relative sea level curves span most of the Holocene transgressive (MIS 1) period for the Gulf of Mexico (Figure 2) (Milliken et al. 2008, Törnqvist et al., 2004a, 2004b, 2006). Climate shifts during the Early Holocene caused punctuated rapid sea level rise episodes (Milliken et al. 2008) and sediment supply variations resulting in periods of rapid shoreline and bayline migration along Texas (Simms et al. 2008, Rodriguez et al. 2004, 2010, Milliken et al. 2008), Mississippi (Flocks 2009), and Alabama (Greene et al. 2007). Sea level rise decreased to about 0.6$0.4 \mathrm{~mm} / \mathrm{yr}$ from about 4,000 years (Milliken et al. 2008) to $1750 \mathrm{CE}$, when it dramatically increased to $1.3-2 \mathrm{~mm} / \mathrm{yr}$ based on Florida saltmarsh records (Gerlach et al. 2017). This abrupt sea level rise trend is apparent within the Gulf (Gerlach et al. 2017) and along the US East Coast (Kemp et al. 2011). Between 1966-2017 CE, current RSLR 
rates are $3.61 \pm 0.59 \mathrm{~mm} / \mathrm{yr}$ at Dauphin Island (NOAA 2018: Station 8735180).

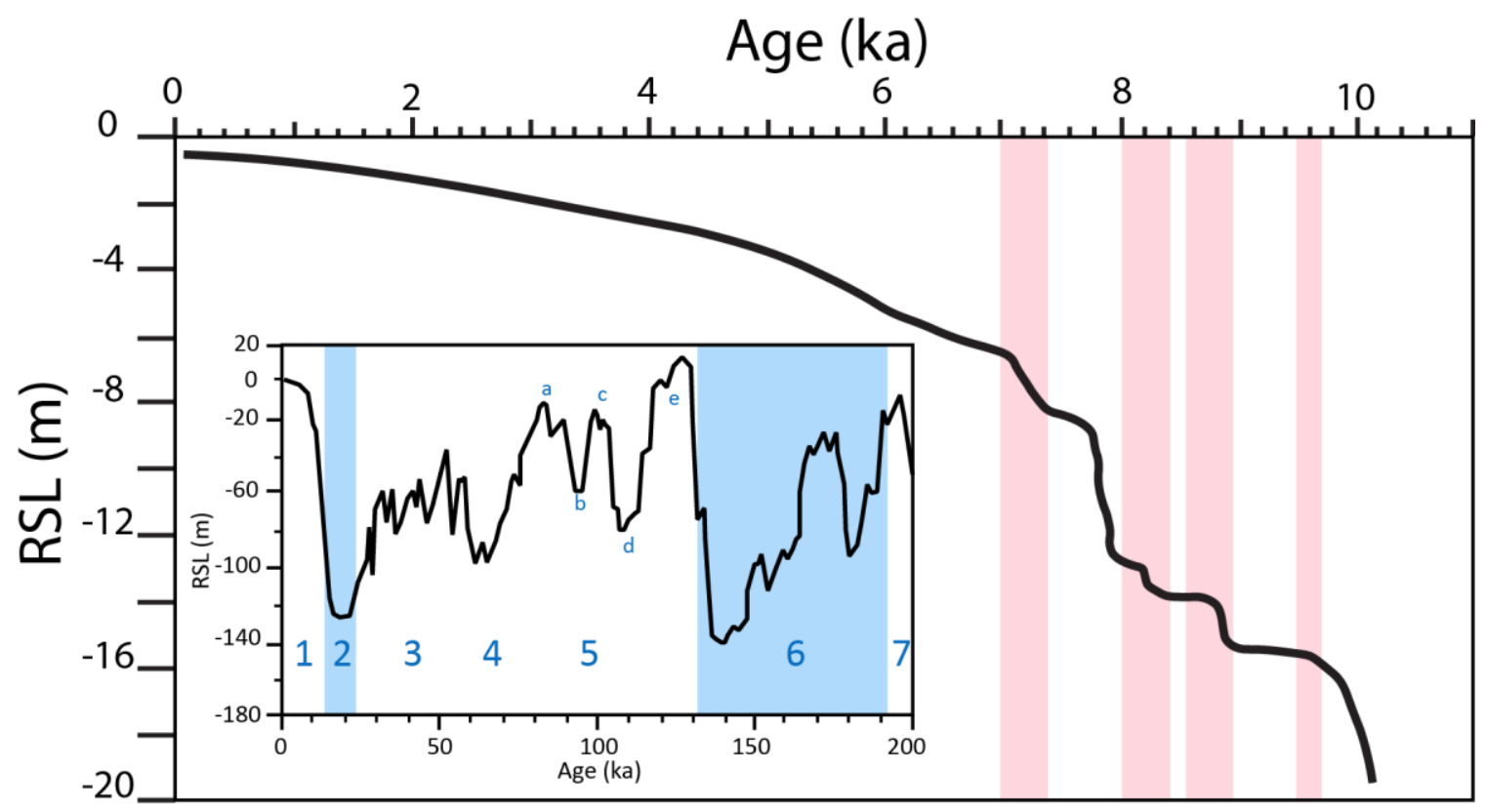

Figure 2 Eustatic and Texas/Louisiana Sea Level Curves

Composite, non-linear, Holocene sea level curve in the Northern Gulf of Mexico (Anderson et al. 2014, modified from Milliken et al. 2008) based on peat and Donax sp. elevations and dates. For full error measurements and methodology see Milliken et al. (2008). Red bars indicate flooding events observed in estuaries along the Gulf of Mexico. Inset figure displays the eustatic sea level curve over the last 200ka based on the Shackleton $(2000,1987)$ data. Blue numbers indicate Marine Isotope Stage (MIS) number.

\subsection{Barrier Formation and Evolution}

The MSAL barrier island chain is unique in that the five islands (Cat, Ship, Horn, Petit Bois and Dauphin Island) have different geomorphologies, sizes and erosion rates (Morton 2008). Otvos (1970, 1981) states the MSAL barrier formed from vertical shoal aggradation atop Holocene marine sandy muds. McBride et al. (1991) argues the islands experienced an early transgressive phase. Petit Bois and Dauphin Island make up the eastern part of the MSAL barrier chain. The eastern portion of Dauphin Island is anchored by a Pleistocene Gulfport Formation barrier remnant, while the western Dauphin spit and the rest of the barriers emerged around 5.7-5.0 ka BP (Figure 3) (Otvos 1979, 1981, 1985, Otvos and Carter 2008). During this time, RSLR rates decelerated from $\sim 1.6 \mathrm{~mm} / \mathrm{yr}$ to less than $\sim 0.6 \mathrm{~mm} / \mathrm{yr}$, aiding island stabilization (Figure 2) (Milliken 
et al. 2008). The islands are thought to be anchored by a roughly east-west trending, antecedent wave-cut escarpment of relatively significant relief formed during a sea level still stand (McBride et al. 1991, Otvos 1981, Swift et al. 1984).

Historical charts from 1752 CE show the eastern strandplain of the modern Petit Bois Island attached to Dauphin Island (Otvos 1985, Morton 2008, Buster and Morton 2011). The island was separated by an unnamed Hurricane between $1740 \mathrm{CE}$ and the 1850's CE (Otvos and Carter 2013) and continued to migrate westward to its modern position. The origins of some islands are impossible to conclude due to reworking by transgressive processes and storms (Otvos 1970). Paleo storm records from western Mississippi, document an active hurricane period between 900-600 BP and 2200- 1900 BP (Bregy et al. 2018). The low elevation MSAL barriers are especially vulnerable to storm impacts, evidenced by Katrina in 2005 (Eisemann et al. 2018), which will only be exacerbated with accelerated sea level rise in the future.

Previous studies state that an eroding pre-Holocene headland located in the Florida Panhandle (Stone et al. 1992) and Mobile ebb tidal sands (Otvos 1979) source the MSAL barrier sands through alongshore currents (Otvos 1979). Only minor attention is given to the contribution of reworked shelf deposits (Otvos 1979, Rucker and Snowden 1990).

\subsection{Shore Oblique Sand Ridges}

The inner shelf surrounding Petit Bois Island is located on the border of St. Bernard prodelta muds and the Mississippi-Alabama-Florida (MAFLA) sand sheet, consisting of numerous post transgressive, shore oblique sand ridges in water depths of 7$20 \mathrm{~m}$ (Flocks 2015, McBride et al. 1991). Offshore of Dauphin Island is a relatively 
featureless shelf between the Petit Bois Pass and the Mobile ebb tidal delta (Figure 1) (Hummel 1999). Sand ridges generally occur in areas of high sediment availability, sufficient current for sediment transport, and some seafloor perturbation that acts as a depositional nucleus (McBride and Moslow 1991). Flocks (2015) attributes the largest, stable ridges (up to $10 \mathrm{~km}$ long, $0.5 \mathrm{~km}$ wide) seaward of Petit Bois Island to the proximal location of a large fluvial valley sand source (Figure 1). This agrees with Browder and McNich (2006) who showed a significant positive correlation between fluvial channel width and ridge size. It remains unclear whether these detached ridges formed as shoreface attached ridges and became separated through various processes such as shoreline transgression (Hayes and Nairn 2004) and convergence of storm flow (Trowbridge 1995), actively translating ebb tidal delta deposits drowning with sea level rise (McBride and Moslow 1991), or if the shoals formed on the inner shelf (Snedden and Dalrymple 1999).

\subsection{Mississippi/Alabama Coastal Geology}

Fluctuating sea levels controlled deposition of several marine-fluvial coastal plain sequences comprising the MSAL coast (Figure 3 ). The 40-60m thick, Late Pliocene age Citronelle Formation represents paralict sand rich alluvial/fluvial to estuarine sequence deposits (Otvos 2001). The Pre-Sangamon terrace represents alluvial deposits luminescence dated at 224-202 ka (MIS 7) (Otvos 2001). 

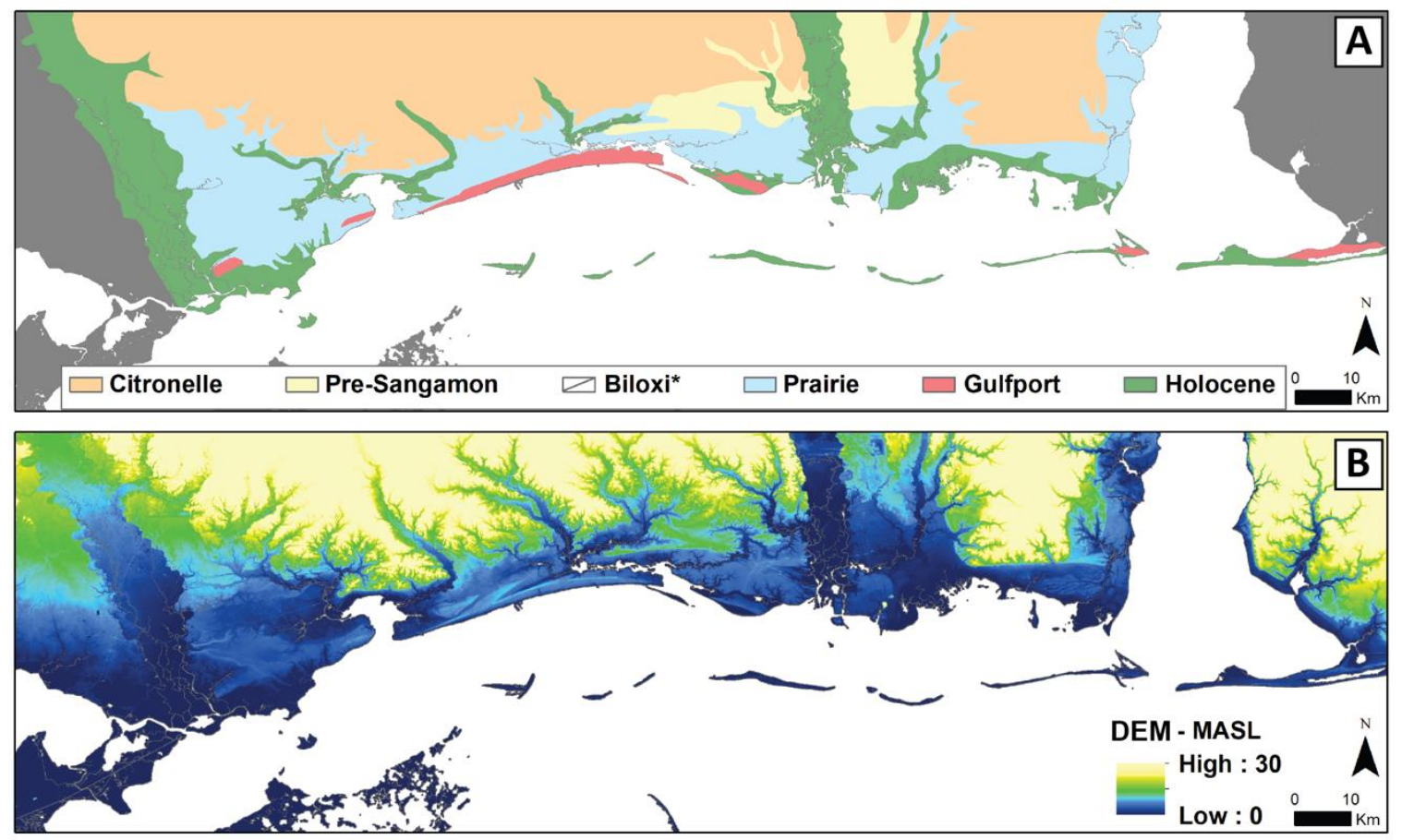

Figure 3 MSAL Coastal Geology and Lidar

The coastal geologic formations of Mississippi and Alabama, ranging from the Early Pliocene to modern deposits, based on the stratigraphic naming convention of Otvos (1985, 2001). The asterisk indicates the Biloxi Formation, which underlies and interfingers with the Prairie and Gulfport Formation. B) Composite 2007 Biloxi, MS and 2009 Mobile, AL DEM topobathy rasters corrected to mean sea level (NOAA 2007, 2009).

The Late Pleistocene units include the alluvial Prairie Formation terrace, the beach ridge Gulfport Formation and the underlying marine to estuarine Biloxi Formation (Otvos 2001). The 3.5-12m thick, pale-yellow to olive-green, clayey sandy Prairie Formation is luminescence dated to 120-90 ka (MIS 5) (Otvos 2005). Lower elevation Prairie terraces in western Mississippi show alluvial deposition continued from 50-30ka (MIS 3) (Otvos 2005). The 3-8m thick, Gulfport Formation represents a nearshore to intertidal to eolian sequence interpreted as barrier ridge and beach deposits (Brown et al. 1944) dating to the MIS 5e highstand (124-115 ka BP) (Otvos 2001). This discontinuous beach ridge deposit is regionally correlated throughout portions of Texas and Louisiana (Ingleside) to the west and Florida to the east. Portions of Dauphin and Fort Morgan Alabama are MIS 5e beach deposits (Otvos 2001). The 4-16m blueish gray muddy sandy 
Biloxi Formation represents a fossil rich estuarine- marine unit. Overlying these Late Pleistocene deposits are a series of thin Holocene transgressive coastal plain deposits ranging from marine to fluvial environments. 


\section{3-METHODS}

\subsection{Geophysical Data}

This project incorporated $\sim 2050 \mathrm{~km}$ and $\sim 950 \mathrm{~km}$ of archived chirp and boomer seismic data, respectively. The chirp data sets (Forde et al. 2011a, 2011b, 2015) were collected from 2008-2013 by the United States Geological Survey (USGS) as part of the Mississippi Coastal Improvements Program (MsCIP). Chirp data from Greene et al. (2007) were also incorporated (Figure 4). Boomer and mini sparker seismic data (Bosse et al. 2017a, 2017b, Sanford et al. 2016a, 2016b, 2016c) were collected from 1981-1992 (Figure 4). See references within figure (4) for survey specific method details. Previously collected data set line spacing, resolution and horizontal accuracy varied greatly, which required weighted quality control between datasets and later interpretation. Chirp data collected with an Edgetech SB-512i had the greatest penetration and detail in shallow water depths and areas of thick sand packages. Other instruments (Edgetech SB-216s or SB-424) provided sufficient detail in muddy portions of Mississippi Sound. Recently collected chirp data was weighted higher overall due to greater vertical resolution $(\leq$ $20 \mathrm{~cm})$ and spatial accuracy $(\leq 2 \mathrm{~m})$ compared to seismic data collected with LORAN-C

$(\leq 1 \mathrm{~m}, \leq 0.4 \mathrm{~km}$ respectively). Seismic data were classified based on the amount of ringing or noise present. Due to certain data coverage limitations, seismic was used where chirp was absent and to delineate deep stratigraphic boundaries.

Seismic data collected during this study were acquired using a single-channel Applied Acoustics boomer with a CSP1000 power supply and Hypack data acquisition software. The analog geophysical trace information was converted to a digital signal 
using a National Instruments conversation system. Navigation was provided by a WASS enabled Trimble DPGS with a horizontal spatial accuracy of $\sim \pm 3 \mathrm{~m}$.

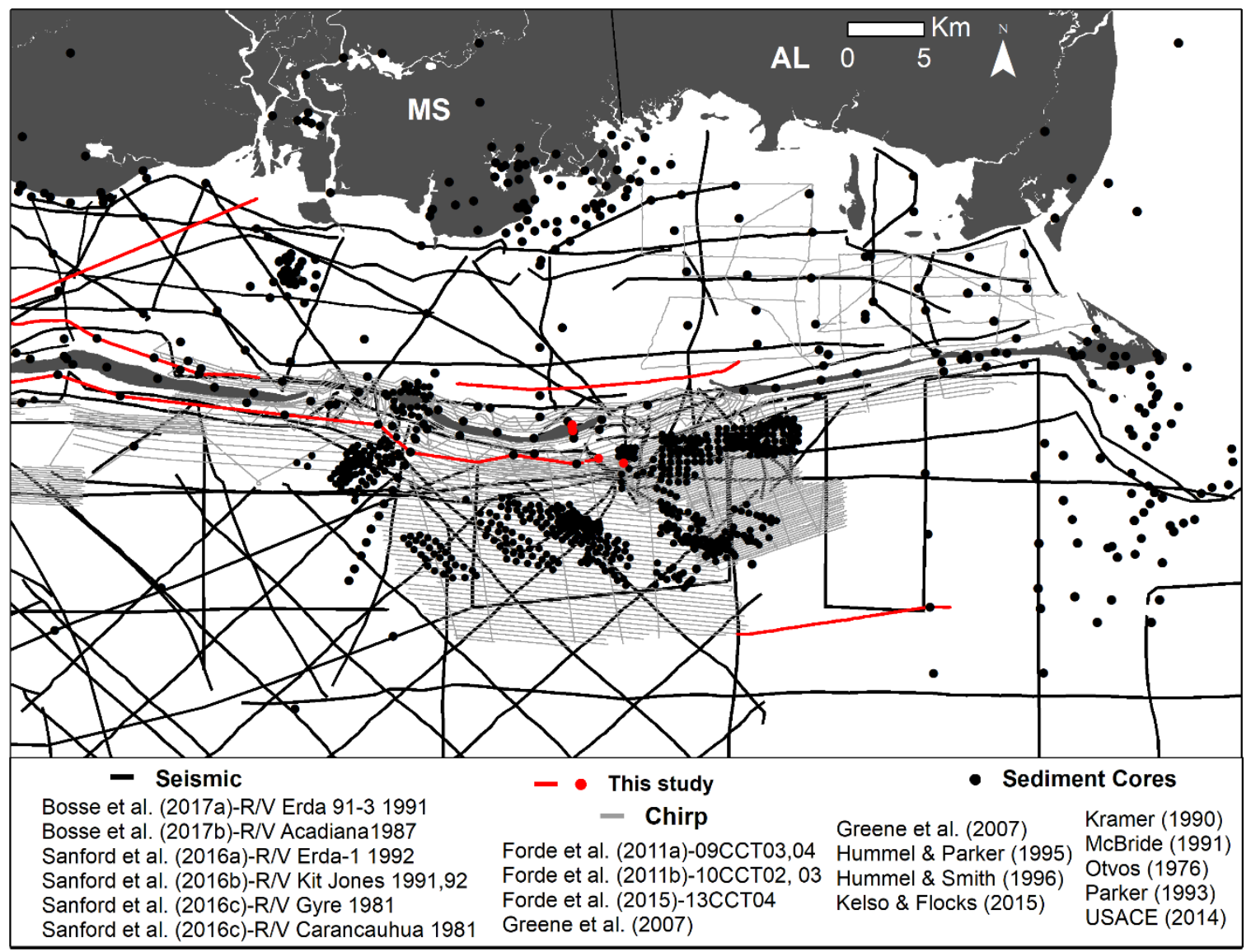

Figure 4 Data Coverage

Synthesized data used in this study from various sources (references within figure). Boomer/minisparker seismic and chirp profiles are represented thick black and grey lines, respectively. Sediment cores are represented by black dots. Data collected in this study are shown in red.

Chirp and seismic SEGY data were analyzed and interpreted in SonarWiz 6 (Chesapeake Technologies, 2018) supplemented with sediment cores. Seismic and lithologic facies were constructed based on stratigraphic relationships following facies models and descriptions proposed by Zaitlin et al. (1994), Mitchum et al. (1977), Allen and Possamentier (1993) and Cataneuo and Steel (2003). These reflector picks were exported as xyz data and then gridded using the kriging function in Golden Software Surfer 15. Separate surfaces with nodal points spaced at $75 \mathrm{~m}$ and $200 \mathrm{~m}$ resolution were 
created and later merged due to the variable chirp and older seismic data densities. A smoothing function and some interpretive surface modifications were made to remove gridding artifacts. Finally, these interpolated surfaces were incorporated into ArcGIS to create paleotopographic maps.

\subsection{Sediment Cores}

This study integrated over 720 previously collected sediment cores (vibracores and rotary drill cores) from numerous sources to create stratigraphic geometries and ultimately an evolutionary model of the MSAL coastal setting (Figure 4). The compiled legacy cores came from nine studies (Figure 4 and references within). Information available ranged from geotechnical sand prospecting logs with limited photographs to archived cores that were available for sampling. Depending on the data quality of the archive core, some were used to simply ground truth the subaerial exposure surface, indicating the Pre-Holocene contact or lowstand sequence boundary. Other archived cores provided more detailed sedimentary structure descriptions used to interpret lithofacies. Two scuba dive assisted percussion cores and four vibracores collected for this study focused on penetrating and dating inner shelf valley fill deposits and Petit Bois Island stratigraphy. These cores were split using normal logging methods. Lithologic units from these cores were analyzed for grain size, sedimentary structure, shell content, contact relationships, and radiocarbon sample type.

\subsection{Radiocarbon Dating Estimates}

New macrofossil samples were collected from archived cores at the USGS Center for Marine and Coastal Science (Kelso and Flocks 2015), Geological Survey of Alabama (Hummel and Parker 1995) and cores collected in this study for radiocarbon dating 
(Table 1). Twenty-four new radiocarbon dates were obtained using the continuous flow gas bench accelerator mass spectrometer method developed at the National Ocean Sciences Accelerator Mass Spectrometry (NOSAMS) of the Woods Hole Oceanographic Institution. Sampling focused on articulated bivalves when possible. Articulated samples were determined to be in situ because following death, the organic ligament joining individual valves decays and becomes very sensitive to currents (Pilarczyk and Barber 2015). Sample preparation followed NOSAMS guidelines. The gas bench ages were calibrated with the Marine13 curve (Reimer et al. 2013) with the standard global 400 year-marine reservoir applied. While there are distinct potential chemical signatures from multiple drainage basins influencing the study area (Milliken et al. 2008, Törnqvist et al. 2015) and potential variations of fractionation locally within a single estuary (Hadden and Cherkinsky 2016, 2017), no additional reservoir correction was made for estuarine samples due to limited data available in the northern-central Gulf of Mexico (Reimer et al. 2001).

Thirty-eight radiocarbon dates from mollusk, peat, wood and other organic sediment samples were synthesized from various literature sources listed in Table 2. Previously published radiocarbon samples were recalibrated using the Marine13 and IntCal13 curves for marine and terrestrial samples, respectively (Reimer et al. 2013). No local reservoir corrections were applied.

All ages (new and recalibrated) are reported in calendar years before present (BP, present being $1950 \mathrm{CE}$ ), using median probability with associated two-sigma ranges. All elevations represent meters referenced to mean sea level. 


\subsection{Sand Volume Calculations}

Methods outlined by Weight et al. (2011) were employed to quantify sediment produced by wave and tidal scour (transgressive ravinement) during the Holocene. This involves multiplying the flooded areal extent of the MIS 2 surface (based on sea level curves from Milliken et al. (2008); Figure 2) by the depth of modern transgressive ravinement. The depth of ravinement was assumed to be constant throughout the Holocene, although it was likely variable based on the geologic framework (sand supply and resistance to erosion), rates of sea level rise, and antecedent topography (Swift et al. 1975). In this study, we assume a conservative depth of wave ravinement (wRs) of 7 meters below sea level (mbsl) based on cores and seismic data showing marine muds onlapping shoreface sands or exposed Pre-Holocene deposits. Dense geophysical data have constrained the footprint of several sandy fluvial or deltaic deposits on the inner shelf that have a clear ravinement surface truncation. Sand content (lithology and extent) was estimated from sediment core and geophysical data. Thus, the methodology from Weight et al. (2011) was employed to find the volume of sediment produced from deposits identified as having high sand content only, not the entire inner shelf.

Calculated sand volumes produced through ravinement were compared to that of the active Holocene sections of Petit Bois Island, Petit Bois Pass and the Dauphin Island western spit platforms. Eastern Dauphin is an older Pleistocene barrier deposit (Figure 3) and excluded from volumetric analysis. For this computation, we have defined the active barrier system as the sand content bounded by the toe of the shoreface (based on cores and geophysical), the base of barrier island and inlet fill sands, to the distal flood tidal delta or overwash extent (Figure 1). The volume of sand was calculated between the 
basal sand and modern topobathymetric (NOAA 2007, 2009) surfaces clipped to the active barrier system extent. For this calculation, the modern barrier system was assumed to be composed of $100 \%$ sand.

\subsection{Evolutionary Model}

Sea level age and elevation points from the Milliken et al. (2008) curve were used to "flood" the MIS 2 lowstand subaerial surface. These data assumed no Holocene sedimentation and allowed for estimation of marine flooding extents and paleo water depths. Environmental reconstructions throughout the Holocene transgression were made from these maps and were constrained by seismic, lithofacies, and radiocarbon data. 


\section{4-RESULTS}

\subsection{Quaternary Chronostragraphic Framework}

Two regional sequence boundaries, several bounding ravinement surfaces and fourteen facies were identified from geophysical and sediment core data. The two sequence boundaries (SB1 and SB2) represent different lowstand, subaerial exposure surfaces with different incisional geometries (Figure 5). Twenty-four new calibrated gas bench radiocarbon ages collected in this study and thirty-eight synthesized radiocarbon dates constrain the evolutionary timing of these facies (Table 1 and 2). The seismic and lithofacies (Table 3) are a culmination of previous work assembled for this study and reinterpreted holistically (Hummel and Parker 1995, Flocks 2015, McBride et al. 1991, Twichell et al. 2011, Kramer 1999, Otvos 1979, 1981 1985, 1986). These seismiclithologic units reveal the preserved portions of the stratigraphic record and help clarify the Quaternary evolution of the area and geologic framework. 
Table 1 New Radiocarbon Ages

\begin{tabular}{|c|c|c|c|c|c|c|c|}
\hline $\begin{array}{l}\text { Study } \\
\text { ID }\end{array}$ & Core ID & $\begin{array}{l}\text { El. } \\
(\mathrm{msl})\end{array}$ & Material Dated & $C^{14}$ Age & $\begin{array}{l}\text { Median } \pm \text { Cal. } \\
2 \sigma \text { Age (yrs BP) }\end{array}$ & $\begin{array}{c}\text { Cal. 2o Age } \\
\text { Range (yrs BP) }\end{array}$ & Facies \\
\hline 1 & $10 \operatorname{cct} 05-02$ & -11.59 & $\begin{array}{l}\text { Mulinia } \\
\text { lateralis }\end{array}$ & $2,210 \pm 120$ & $1,808 \pm 289$ & $1,526-2,104$ & U Shoreface \\
\hline 2 & $10 \operatorname{cct} 05-02$ & -12.61 & $\begin{array}{l}\text { Mulinia } \\
\text { lateralis }\end{array}$ & $2,840 \pm 160$ & $2,570 \pm 393$ & $2,162-2,947$ & L Shoreface \\
\hline 3 & $10 \operatorname{cct} 05-02$ & -13.61 & Raeta plicatella & $6,500 \pm 120$ & $7,001 \pm 274$ & $6,712-7260$ & U Shoreface \\
\hline 4 & $10 \operatorname{cct} 05-03$ & -14.11 & Rangia flexuosa & $6,420 \pm 120$ & $6,907 \pm 283$ & $6,629-7,195$ & Estuarine \\
\hline 5 & $10 \operatorname{cct} 05-03$ & -15.80 & $\begin{array}{c}\text { Crassostrea } \\
\text { virginica } *\end{array}$ & $>38,900$ & - & - & Estuarine \\
\hline 6 & $10 \operatorname{cct} 05-3$ & -15.8 & $\begin{array}{c}\text { Noetia } \\
\text { ponderosa }\end{array}$ & $>40,900$ & - & - & Estuarine \\
\hline 7 & $10 \operatorname{cct} 05-3$ & -15.91 & $\begin{array}{c}\text { Mercenaria } \\
\text { campechiensis* }\end{array}$ & $>41,000$ & - & - & Marine \\
\hline 8 & $10 \operatorname{cct} 05-06$ & -5.14 & $\begin{array}{l}\text { Cyclinella } \\
\text { tenius }\end{array}$ & $930 \pm 100$ & $537 \pm 229$ & $334-681$ & Flood Tidal Delta \\
\hline 9 & $10 \operatorname{cct} 05-06$ & -5.64 & Strombus alatus & $2,610 \pm 110$ & $2,295 \pm 313$ & $2,019-2,644$ & Flood Tidal Delta \\
\hline 10 & $10 \operatorname{cct} 05-07$ & -5.79 & Abra aquelis & >Modern & - & - & Estuarine \\
\hline 11 & $10 \operatorname{cct} 05-07$ & -6.10 & $\begin{array}{l}\text { Mulinia } \\
\text { lateralis }\end{array}$ & >Modern & - & - & Estuarine \\
\hline 12 & $10 \operatorname{cct} 05-07$ & -6.11 & $\begin{array}{l}\text { Mulinia } \\
\text { lateralis }\end{array}$ & $115 \pm 100$ & - & - & Estuarine \\
\hline 13 & $10 \operatorname{cct} 05-08$ & -5.37 & Abra aquelis & $920 \pm 100$ & $529 \pm 171$ & $329-671$ & Estuarine \\
\hline 14 & PBO17-2 & -11.11 & Abra aquelis & $2,480 \pm 110$ & $2,135 \pm 268$ & $1,855-2,390$ & Estuarine \\
\hline 15 & MS1 & -3.55 & Shell fragments & $2,940 \pm 110$ & $2,702 \pm 280$ & $2,376-2,953$ & Flood Tidal Delta \\
\hline 16 & MS1 & -4.53 & Shell fragments & $4,070 \pm 140$ & $4,070 \pm 381$ & $3,725-4,486$ & Flood Tidal Delta \\
\hline 17 & MS5 & -3.04 & $\begin{array}{c}\text { Cyclinella } \\
\text { tenius }\end{array}$ & $1,650 \pm 110$ & $1,199 \pm 232$ & $949-1,412$ & Estuarine \\
\hline 18 & MS5 & -3.69 & $\begin{array}{l}\text { Trachycardium } \\
\text { sp. }^{\text {a }}\end{array}$ & $2,720 \pm 110$ & $2,450 \pm 274$ & $2,166-2,714$ & Estuarine \\
\hline 19 & MS5 & -4.07 & Shell frag & $3,680 \pm 110$ & $3,600 \pm 296$ & $3,319-3,911$ & Estuarine \\
\hline 20 & MS9 & -3.93 & $\begin{array}{l}\text { Mulinia } \\
\text { lateralis }\end{array}$ & Modern & - & - & Estuarine \\
\hline 21 & MS9 & -3.98 & $\begin{array}{l}\text { Mercenaria } \\
\text { Mercenaria }\end{array}$ & Modern & - & - & Marine/ FTD \\
\hline 22 & MS9 & -4.88 & Abra aquelis & $400 \pm 100$ & - & - & $\begin{array}{c}\text { Barrier } \\
\text { (overwash) }\end{array}$ \\
\hline $23 \mathrm{a}$ & MS9 & -5.85 & $\begin{array}{c}\text { Cyrtopleaura } \\
\text { costata }\end{array}$ & $2,150 \pm 110$ & $1,738 \pm 265$ & $1,475-2,005$ & Estuarine \\
\hline $23 b$ & MS9b & -5.85 & $\begin{array}{c}\text { Cyrtopleaura } \\
\text { costata }\end{array}$ & $2,120 \pm 110$ & $1,703 \pm 265$ & $1,433-1,962$ & Estuarine \\
\hline
\end{tabular}

Gas bench radiocarbon dates produced from this study. Marine samples were calibrated using the Marine13 curve (Reimer et al. 2013). No local reservoir corrections were made. All dated samples were in situ, intact, articulated bivalves, except sample 9 (gastropod) and samples with a, indicating one single intact valve or reworked samples. * notes sample beyond the radiocarbon detection limit. Elevation is in meters referenced to sea level. All dates discussed in text are reported as in years before present (BP, present being $1950 \mathrm{CE}$ ), median calendar ages with added two sigma ranges. 
Table 2 Synthesized Radiocarbon Ages

\begin{tabular}{|c|c|c|c|c|c|c|c|c|}
\hline $\begin{array}{l}\text { Study } \\
\text { ID }\end{array}$ & Core ID & El. (msl) & $\begin{array}{c}\text { Material } \\
\text { Dated }\end{array}$ & $\mathrm{C}^{14}$ Age & $\begin{array}{c}\text { Median } \pm \text { Cal. 2o } \\
\text { Age (yrs BP) }\end{array}$ & $\begin{array}{c}\text { Cal. 2o Age } \\
\text { Range (yrs BP) }\end{array}$ & Facies & Citation \\
\hline 24 & DI-1 & -12.30 & Peat $^{\mathrm{b}}$ & $23,500 \pm 3,825$ & $27,434 \pm 8,168$ & $18,875-35,211$ & Estuarine & Otvos (1979) \\
\hline 25 & DI-2 & -11.40 & Wood $^{\text {b }}$ & $25,840 \pm 1,330$ & $29,887 \pm 2,606$ & $27,433-32,645$ & Estuarine & Otvos (1979) \\
\hline 26 & DI-2 & -11.90 & Wood $^{\text {b }}$ & $36,290 \pm 2,060$ & $40,434 \pm 3,957$ & $36,103-44,018$ & Estuarine & Otvos (1979) \\
\hline 27 & DI-2 & -13.20 & Wood $^{\text {b }}$ & $30,400 \pm 2,185$ & $34,506 \pm 4,579$ & $30,254-39,412$ & Estuarine & Otvos (1979) \\
\hline 28 & DI-3 & -2.90 & Mollusk & $6,670 \pm 165$ & $7,175 \pm 348$ & $6,795-7,490$ & Inlet Fill/Spit & Otvos (1979) \\
\hline 29 & DI-6 & -2.57 & Oyster Shell a & $1,215 \pm 80$ & $798 \pm 145$ & $654-943$ & Flood Tidal Delta & Otvos (1986) \\
\hline 30 & DI-8 & -0.20 & Humate & $7,700 \pm 820$ & $8,665 \pm 1,874$ & $6,848-10,596$ & Marsh & Otvos (1979) \\
\hline 31 & P-3 & -13.63 & Wood & $7,815 \pm 80$ & $8,613 \pm 187$ & $8,420-8,793$ & Estuarine & Otvos (1979) \\
\hline 32 & P-4 & -18.00 & $\begin{array}{l}\text { Strombus } \\
\text { alatus }^{\text {a }}\end{array}$ & $38,960 \pm 3,125$ & $42,430 \pm 6,029$ & $36,162-48,219$ & Marine Mud & Otvos (1979) \\
\hline 33 & $\mathrm{R}-1$ & -11.89 & Wood & $7,315 \pm 85$ & $8,127 \pm 174$ & $7,975-8,323$ & Estuarine & Otvos (1979) \\
\hline 34 & R-3 & -8.40 & $\begin{array}{l}\text { Dispersed } \\
\text { plant }\end{array}$ & $6,765 \pm 270$ & $7,635 \pm 511$ & $7,155-8,176$ & Estuarine & Otvos (1979) \\
\hline 35 & $\mathrm{R}-3$ & -15.40 & Wood & $7,825 \pm 160$ & $8,678 \pm 346$ & $8,342-9,034$ & Estuarine & Otvos (1979) \\
\hline 36 & $\mathrm{H}-1$ & -11.10 & Cardium sp. & $4,615 \pm 215$ & $4,839 \pm 567$ & $4,284-5,418$ & Barrier & Otvos (1979) \\
\hline 37 & $\mathrm{H}-2$ & -16.20 & Peat & $8,010 \pm 85$ & $8,865 \pm 244$ & $8,604-9,092$ & Marsh (fresh?) & Otvos (1979) \\
\hline 38 & $\mathrm{H}-7$ & -18.60 & Wood & $34,935 \pm 1820$ & $39,202 \pm 3526$ & $35,400-42,451$ & MIS2 estuarine & Otvos (1979) \\
\hline 39 & SS-4 & -15.59 & $\begin{array}{l}\text { Strombus } \\
\text { alatus }\end{array}$ & $6,315 \pm 80$ & $6,779 \pm 156$ & $6,582-6,894$ & Marine Mud & Otvos (1985) \\
\hline
\end{tabular}


Table 2 Continued

\begin{tabular}{|c|c|c|c|c|c|c|c|c|}
\hline $\begin{array}{c}\text { Study } \\
\text { ID }\end{array}$ & Core ID & El. (msl) & $\begin{array}{c}\text { Material } \\
\text { Dated }\end{array}$ & $\mathrm{C}^{14}$ Age & $\begin{array}{c}\text { Median } \pm \text { Cal. 2o } \\
\text { Age (yrs BP) }\end{array}$ & 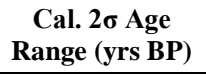 & Facies & Citation \\
\hline 40 & SS-4 & -16.50 & Oyster Shell ${ }^{\text {a }}$ & $21,640 \pm 4,690$ & $24,827 \pm 10,440$ & $13,722-34,602$ & Estuarine & Otvos (1985) \\
\hline 41 & SS-8 & -15.44 & Mollusk & $4,160 \pm 305$ & $4,223 \pm 793$ & $3,438-5,023$ & Marine Mud & Otvos (1985) \\
\hline 42 & SS-8 & -18.17 & $\begin{array}{l}\text { Strombus } \\
\text { alatus }\end{array}$ & $4,735 \pm 115$ & $5,004 \pm 299$ & $4,704-5,302$ & Marine Mud & Otvos (1985) \\
\hline 43 & SS-10 & -18.47 & $\begin{array}{l}\text { Cyrtopleura } \\
\text { costata }\end{array}$ & $4,865 \pm 175$ & $5,153 \pm 407$ & $4,768-5,581$ & Estuarine? & Otvos (1985) \\
\hline 44 & SS-11 & -21.20 & Wood & $8,800 \pm 80$ & $9,849 \pm 282$ & $9,595-10,158$ & Estuarine & Otvos (1985) \\
\hline 45 & SS-12 & -19.68 & $\begin{array}{l}\text { Mercenaria } \\
\text { m. texana }\end{array}$ & $6,560 \pm 95$ & $7,070 \pm 210$ & $6,847-7,266$ & Sand Sheet & Otvos (1985) \\
\hline 46 & SS-12 & -21.20 & $\begin{array}{c}\text { Mercenaria } \\
\text { campechiensis } \\
\mathrm{b}\end{array}$ & $6,400 \pm 75$ & $6,879 \pm 203$ & $6,686-7,092$ & Flood Tidal Delta & Otvos (1985) \\
\hline 47 & MS-9 & -7.12 & Peat & $6,590 \pm 60$ & $7,491 \pm 80$ & $7,420-7,580$ & Marsh & Hummel and Parker (1995) \\
\hline 48 & MS-11 & -7.57 & $\begin{array}{l}\text { Organic } \\
\text { Sediment }\end{array}$ & $6,040 \pm 150$ & $6,905 \pm 359$ & $6,544-7,261$ & Marsh & Hummel and Parker (1995) \\
\hline 49 & MS-13 & -4.54 & Wood (root) & $6,860 \pm 100$ & $7,710 \pm 157$ & $7,565-7,878$ & Estuarine/Delta & Hummel and Parker (1995) \\
\hline 50 & MS-14 & -6.06 & Peat & $6,190 \pm 80$ & $7,086 \pm 191$ & $6,887-7,269$ & Marsh & Hummel and Parker (1995) \\
\hline 51 & MS-15 & -3.85 & Oyster Shell & $3,700 \pm 60$ & $3,618 \pm 169$ & $3,460-3,798$ & Oyster Biostrome & Hummel and Parker (1995) \\
\hline 52 & MS-24 & -4.15 & Oyster Shell & $3,570 \pm 70$ & $3,465 \pm 172$ & $3,301-3,644$ & Oyster Biostrome & Hummel and Parker (1995) \\
\hline 53 & MS-25 & -3.82 & Oyster Shell & $2,690 \pm 60$ & $2,401 \pm 193$ & $2,262-2,648$ & Oyster Biostrome & Hummel and Parker (1995) \\
\hline 54 & MS-27 & -2.48 & Wood & $4,020 \pm 110$ & $4,512 \pm 300$ & $4,230-4,830$ & Estuarine & Hummel and Parker (1995) \\
\hline
\end{tabular}


Table 2 Continued

\begin{tabular}{|c|c|c|c|c|c|c|c|c|}
\hline $\begin{array}{l}\text { Study } \\
\text { ID }\end{array}$ & Core ID & El. (msl) & $\begin{array}{c}\text { Material } \\
\text { Dated }\end{array}$ & $\mathrm{C}^{14}$ Age & $\begin{array}{l}\text { Median } \pm \text { Cal. 2o } \\
\text { Age (yrs BP) }\end{array}$ & $\begin{array}{c}\text { Cal. 2б Age } \\
\text { Range (yrs BP) }\end{array}$ & Facies & Citation \\
\hline 55 & MS-28 & -1.64 & Oyster Shell & $710 \pm 60$ & $359 \pm 108$ & $257-473$ & Oyster biostrome & Hummel and Parker (1995) \\
\hline 56 & MS-28 & -4.27 & Wood & $19,540 \pm 220$ & $2,3531 \pm 539$ & $22,972-24,049$ & MIS2 alluvial & Hummel and Parker (1995) \\
\hline 57 & MS-29 & -5.03 & Oyster Shell & $3,900 \pm 80$ & $3,877 \pm 225$ & $3,644-4,093$ & Estuarine & Hummel and Parker (1995) \\
\hline 58 & MS-04-1 & -7.76 & $\begin{array}{c}\text { Probythenella } \\
\text { lousianae }\end{array}$ & $4,030 \pm 30$ & $4,046 \pm 112$ & $3,935-4,159$ & Estuarine & Greene et al. (2007) \\
\hline 59 & MS-04-1 & -8.66 & Wood* & $>48,000$ & - & - & MIS2 alluvial/estuarine & Greene et al. (2007) \\
\hline 60 & MS-04-1 & -10.52 & Wood* & $>48,000$ & - & - & MIS2 alluvial/estuarine & Greene et al. (2007) \\
\hline 61 & MS-04-4 & -5.91 & Peat & $5,470 \pm 50$ & $6,272 \pm 106$ & $6,184-6,396$ & Marsh & Greene et al. (2007) \\
\hline 62 & MS-04-5 & -12.58 & Wood* & $38,400 \pm 330$ & $42,475 \pm 460$ & $42,017-42,937$ & MIS2 alluvial/estuarine & Greene et al. (2007) \\
\hline
\end{tabular}

Recalibrated, previously published radiocarbon dates from the study area. Marine samples were calibrated using the Marine13 curve (Reimer et al. 2013), Terrestrial samples (peat and wood) were calibrated using the Intcal13 curve (Reimer et al. 2013). No local reservoir corrections were made. a notes previously reported reworked samples, b notes previously reported contaminated age samples, and * notes sample beyond radiocarbon detection limit. Elevation is in meters referenced to sea level. All dates discussed in text are reported in years before present (BP, present being $1950 \mathrm{CE})$ median calendar ages with associated two sigma ranges. 
Table 3 Lithofacies Summary Table

\begin{tabular}{|c|c|c|c|c|c|}
\hline $\begin{array}{c}\text { Interpreted } \\
\text { Facies }\end{array}$ & Sedimentology & Macrofossils & Seismic Facies Characteristics & $\begin{array}{c}\text { Geophysical Example } \\
\end{array}$ & $\begin{array}{c}\text { Facies Association (Overlies:: Lateral:: Bounding } \\
\text { Surfaces) }\end{array}$ \\
\hline $\begin{array}{c}\text { Sand Ridge } \\
\text { (Modern) }\end{array}$ & $\begin{array}{l}\text { Tan to dark gray, massive to planar laminated M-C sand, graded } \\
\text { shelly beds base, few mud filled burrows. Sharp basal lag } 1\end{array}$ & $\begin{array}{l}\text { Echnoid spines, sand dollar frag, heavily yragmented bivalves and } \\
\text { gastropods } 1,2\end{array}$ & $\begin{array}{l}\text { Low to med amplitude, low frequency, transparent to } \\
\text { dipping reflectors. Moundede dxetral geometry with } \\
\text { erosional base } 3,4\end{array}$ & $\begin{array}{l}\text { Tnner Shelf Muds/ } \\
\text { Sand Sheet }\end{array}$ & $\begin{array}{l}\text { Sand Sheet, Inner Shell Mud, Upper/ Lower Shoreface :: } \\
\text { Sand SSeet, IIner Shelf Mud, Ebb Tidal Delta: : sRs }\end{array}$ \\
\hline $\begin{array}{l}\text { Sand Sheet } \\
\text { (Modern) }\end{array}$ & $\begin{array}{l}\text { Tan, massiviv to crudely planar laminated, F-M shelly quartz sand, } \\
\text { some mud filled burrows. Sharp lag at base } 1,5\end{array}$ & $\begin{array}{l}\text { Sand dollar, Atrina serratat, Parvilucina multilineata, Dosinia } \\
\text { discus. Gemma sp., Strombus alatus, Chione sp., Anadara sp. } 2\end{array}$ & $\begin{array}{l}\text { Low to med amplitude, low frequency parrallel to sub } \\
\text { parrallel sheet like reflectors } 3,4\end{array}$ & & $\begin{array}{l}\text { Inner Shelf Mud, Ebb tidal or Estuarine Deposits :: Inner Sand } \\
\text { Sheet, Ebb tidal Delta :: wRs }\end{array}$ \\
\hline Inner Shelf Muds & $\begin{array}{l}\text { Greenish gray, massive biotrubated clean to sandy muds, low } \\
\text { organic content } 6\end{array}$ & $\begin{array}{l}\text { Dosinia a discus, Gemma Gemma, Chione Cancellatata, Mercenaria } \\
\text { mercenaria, corbula sp., anadara transversa, noetia ponderosa } 2\end{array}$ & $\begin{array}{l}\text { Wavy/horizontal paralallil reflectors or transparent. Sheet } \\
\text { drape or basin/channel fill like, onlapping ext. geometry } 6\end{array}$ & & 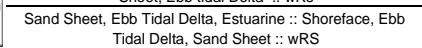 \\
\hline $\begin{array}{c}\text { Barrier System } \\
\text { (Modern) }\end{array}$ & $\begin{array}{l}\text { Washover- Light gray massive to planar laminated sands, } \\
\text { interbedded sands and muds. Dune-well sorted F-VF rooted cross } \\
\text { bedded sands } 7\end{array}$ & $\begin{array}{l}\text { Donax sp., Mulinia lateralis, Lucina multineata, Cyclinella tenuis, } \\
\text { Dosinia discus } 2\end{array}$ & 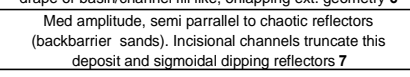 & $\begin{array}{c}\text { mostly subaerial feature with only minor subaqueous } \\
\text { extent }\end{array}$ & $\begin{array}{c}\text { Flood Tidal Delta, Marsth, Estuarine, MIIS S SB, Barrier Spit :: } \\
\text { Flood and Ebb Tidal Dettas, Shorefece, Estuarine, Marsh :: } \\
\text { bRs, tRS, and/or TS }\end{array}$ \\
\hline \multirow{3}{*}{$\begin{array}{l}\text { Upper / Lower } \\
\text { Shoreface }\end{array}$} & \multirow{3}{*}{$\begin{array}{l}\text { Gray-tan, sometimes interbedded mud and sand, clayey sand, sand } \\
\text { and clay pockets, muddy sand dominated upper, shoreface and mud } \\
\text { dominated lower shorofece, wood, rip up clasts, peat balls, wood } \\
\text { and plant material, sand and mud filled burrows. Sharp to } \\
\text { gradational base } 8\end{array}$} & \multirow{3}{*}{ 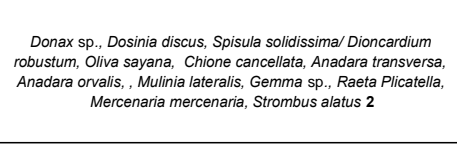 } & \multirow{3}{*}{$\begin{array}{l}\text { Low to med amplitude, med frequency, semi laminated } \\
\text { refflectors. Sheet or wedge ext. geometry } 8\end{array}$} & 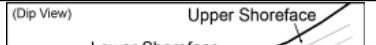 & \multirow{3}{*}{$\begin{array}{l}\text { Estuarine, Flood(?) and Ebb Tidal Delta, Marsh, MIS } 2 \text { SB : } \\
\text { Barrier/ Barrier spit, Ebb Tidal Delta, Inner Shelf muds, San } \\
\text { Ridge, Sand Sheet: : tRs, wRs, TS }\end{array}$} \\
\hline & & & & & \\
\hline & & & & Deposits & \\
\hline $\begin{array}{l}\text { Ebb Tidal Detta } \\
\text { (Modern) }\end{array}$ & 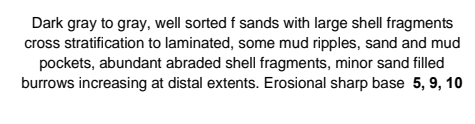 & 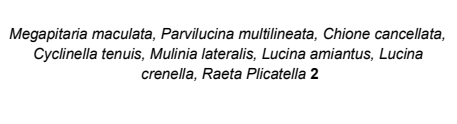 & $\begin{array}{l}\text { Low angle, seaward dipping oblique reflectors. Poor- } \\
\text { moderarty continuous. Downlap underlying surface. Slight } \\
\text { resosive base. Basin fill or lobate ext. geometry } 9 \text {, } 10\end{array}$ & Delta & $\begin{array}{l}\text { Estuarine, Shoreface, MIS } 2 \text { SB:: Shoreface, Sand Sheet, } \\
\text { Inner Shelf Muds, Sand Ridge : : RRS, TS }\end{array}$ \\
\hline $\begin{array}{l}\text { Tidal Inlet/ Barrier } \\
\text { Spit }\end{array}$ & $\begin{array}{l}\text { Inlet- gray, massive to cross bedded (planara and trough) quartz } \\
\text { sands near base, coarse abraded shell laga at base, grainsize and } \\
\text { structure fining upwards. Spiti- low angle planar cross bedded } \\
\text { (wedge sets) with parrallel lamianations of shell and F-M Sand 7, 10, } \\
13\end{array}$ & $\begin{array}{l}\text { Parvilucina multilineatat, Abra aequalis, Cyclinella tenuis, Mulinia } \\
\text { laterallis, Mercenaria mercenaria, Lucina amiantus, Lucina crenella } \\
\text {, Raeta Plicatella, Anadara transversa } 2\end{array}$ & $\begin{array}{l}\text { Unidiriectional, sigmoidal dipping reflectors, rossive base. } \\
\text { Cut and fill, atererally accreting geoometry } 7,10,13\end{array}$ & aterally Accreting S Sit & 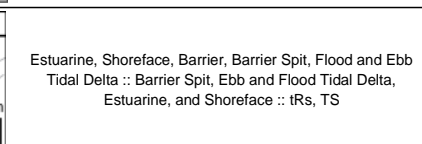 \\
\hline Flood Tidal Delta & $\begin{array}{l}\text { Brownish gray, laminated to massive silty VF- F sand, some mud } \\
\text { ripples, sand and mud couplets, abundant abraded shell fragments, } \\
\text { minor sand filled burrows. Erosional sharp base } 5,9\end{array}$ & Lucina crenella, Chione cancellata 2 & $\begin{array}{l}\text { Dip: Iow angle, landward bidirectional dipping oblique } \\
\text { rerlectors.Striteke horizontal lobatet feature. Poorly continous. } \\
\text { lobate ext. geometry (Simms etal 2010). Proximal to inlet: } \\
\text { channel stacking, cut and fil and lateral accretion. Slight } \\
\text { erosive base 9, } 10\end{array}$ & $\begin{array}{l}\text { (Dip Viewl) } \\
\text { (Landward) }\end{array}$ & $\begin{array}{l}\text { Estuarine, MIS } 2 \text { SB :. Shoreface, Sand Sheet, Inner Shelf } \\
\text { Muds, Sand Ridge:: tRS, TS }\end{array}$ \\
\hline \multirow[t]{2}{*}{ Estuarine Deposits } & $\begin{array}{l}\text { Gray, laminated or heavily bifturbated organic sity clay. Some silty } \\
\text { sand and sandy silt layers, lenticular bedding, sand and mud } \\
\text { sockets. Sandier near its perimeter. Articulated mollusk and }\end{array}$ & 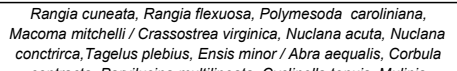 & $\begin{array}{l}\text { Wavy/horizontal parralell ret } \\
\text { drape or basin/channel fill like }\end{array}$ & Estuarine Fill & \multirow{2}{*}{ 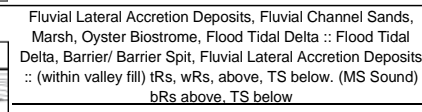 } \\
\hline & $\begin{array}{c}\text { gastropod shells, shell and wood frag, mod } \\
\qquad, 5,11,12\end{array}$ & & & & \\
\hline Oyster Biostrome & $\begin{array}{l}\text { Non-cemented, articulated shells beds and inarticulated shells, } \\
\text { framework supported with matrix of muddy sand and sand } 11\end{array}$ & $\begin{array}{c}\text { Crassostrea virginica, Brachidontes recurvus/ Anomia simplex, } \\
\text { Brachidontes exustus, Ostrea equestris } 2\end{array}$ & $\begin{array}{l}\text { Mounded refilectors low continuity, onlapping proximal } \\
\text { reflectors } 14\end{array}$ & & Fluvial Lateral Accretion Deposits, Estuarine :: Conformable \\
\hline Marsh & $\begin{array}{l}\text { Brown to dark gray peat or rooted zones, organic silty sand matrix, } \\
\text { mootled coloration, mud fililed burrows } 111\end{array}$ & Geukensia demissa, Littorina irrorata (minor) 2 & $\begin{array}{l}\text { Wavy parrallel reflects. Gas masking low continuity Sheet } \\
\text { drape ext. geometry } 12\end{array}$ & & 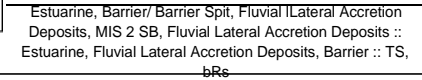 \\
\hline $\begin{array}{l}\text { Fluvial Lateral } \\
\text { Accretion Deposits }\end{array}$ & 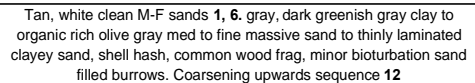 & $\begin{array}{l}\text { Absent of shells to Rangia cuneata, Rangia flexuosa, Tagellus } \\
\text { plebeius, Macoma mitchelli } 2\end{array}$ & $\begin{array}{l}\text { Dipping reflectors, prograding into valley } 6 \text { Toplapping semi } \\
\text { horizontal reflectors in upper section. Slight erosive base. } \\
\text { Basin or channel fill ext. geometry } \mathbf{3 , 6 , 1 2}\end{array}$ & $\begin{array}{l}\text { (Strkike hew) Fluvial Lateral Accretion Deposits } \\
\text { SB }\end{array}$ & $\begin{array}{l}\text { MIIS } 2 \text { SB, Fluvial Channel Sands!: : Estuarine, Fluvial } \\
\text { Lateral Accretion Deposits :: SB below, TS above }\end{array}$ \\
\hline $\begin{array}{c}\text { Fluvial Channel } \\
\text { Sands }\end{array}$ & $\begin{array}{l}\text { Tan, white mod mudddy sands,s, sands, and gravels with } \\
\text { unidirectional, high angle cross strat. heavy mineral grains } 1\end{array}$ & Rangia cuneata (proximal to estuary), Rangia flexuosa 2 & $\begin{array}{l}\text { Chaotic to transparent or dipping oblique reflectors. Channel } \\
\text { fill geometry } 4,6\end{array}$ & & $\begin{array}{l}\text { MIS } 2 \text { SB, Fluvial Channel Sands : : MIS } 2 \text { ::SB below, TS } \\
\text { above }\end{array}$ \\
\hline MIS 2 and older & $\begin{array}{l}\text { Stitf, oxidized, yellowish orange, brown and greenish gray clayey } \\
\text { sand, sandy clay, clay, burrows, some to absent shells, plant } \\
\text { material common, paleosol developopment } 5,6,8,11,12\end{array}$ & Estuarine, deltaic, fluvial alluvial assemblages & $\begin{array}{l}\text { Continuuus reflector boundary, rrosional with variable } \\
\text { confifurations below. Refiectors onlap this surface } 12\end{array}$ & (1) & Underlies MIS2 SB \\
\hline
\end{tabular}

Lithofacies and bounding surfaces group results summary. Facies descriptions (bold numbers) were based on the following studies: 1 Parker et al. (1993), 2 Parker (1960), 3 Flocks (2015), 4 Goff et al. (2014), 5 McBride et al. (1991), 6 Thomas and Anderson (1994), 7 Moslow and Tye (1985), 8 Rodriguez et al. (2001), 9 Siringan and Anderson (1993), 10 Miner et al. (2009), 11 Hummel and Parke (1996), 12 Greene et al. (2007), 13 Simms et al. (2006), 14 Goff et al. (2015). SB=Sequence Boundary, TS= Transgressive Surface, tRs=Tidal Ravinement Surface, wRs Wave Ravinement Surface, bRs= Bay Ravinement Surface, sRS=Sand Ridge Ravinement Surface. 


\subsubsection{Sequence Boundary 1: Marine Isotope Stage 6}

Sequence Boundary 1 (SB1) is characterized by a medium to high amplitude, semi to highly continuous reflector and was mapped 10-45 mbsl (4-25 meters below seafloor (mbsf)). This surface is characterized by incisional channel features that truncate underlying reflectors below with onlapping reflectors above. SB1 likely represents an earlier exposure surface associated with the MIS 6 lowstand (Figure 2 and 5). The larger, wider valleys associated with this lowstand boundary are truncated by another regional sequence boundary (SB2) south of Horn Island Pass. Four dated samples from this stratigraphically deeper valley surface were beyond the radiocarbon detection limit (Table 1 and 2, sample 5, 6, 7 and 62) so an absolute age constraint is lacking. However, the ages pre-date the MIS 2 lowstand. Previous studies working in the area have reported the presence of a similar Pre-MIS 2 surface (Greene et al. 2007, Gonzalez et al. 2017). Roberts et al. (2004) dates the shelf edge Lagniappe Delta to be of MIS 6 or 8 age.

Modern Lidar (NOAA 2007, 2009) shows a large valley updip in the Grand Bay area correlating to the mapped SB1 valley (Figure 1 and 5). According to Otvos (2001) the valley is filled with Prairie Formation alluvial deposits (Figure 3). Otvos (2005) dated the Prairie Formation fill to be $\sim 120 \mathrm{ka}$, which suggests the valley is at least this old. Based on sequence stratigraphy, the most recent lowstand (MIS 6) before MIS 5 produced this valley (Green et al. 2007). Without an absolute age and based on these arguments we assume SB1 represents the MIS 6 lowstand. 

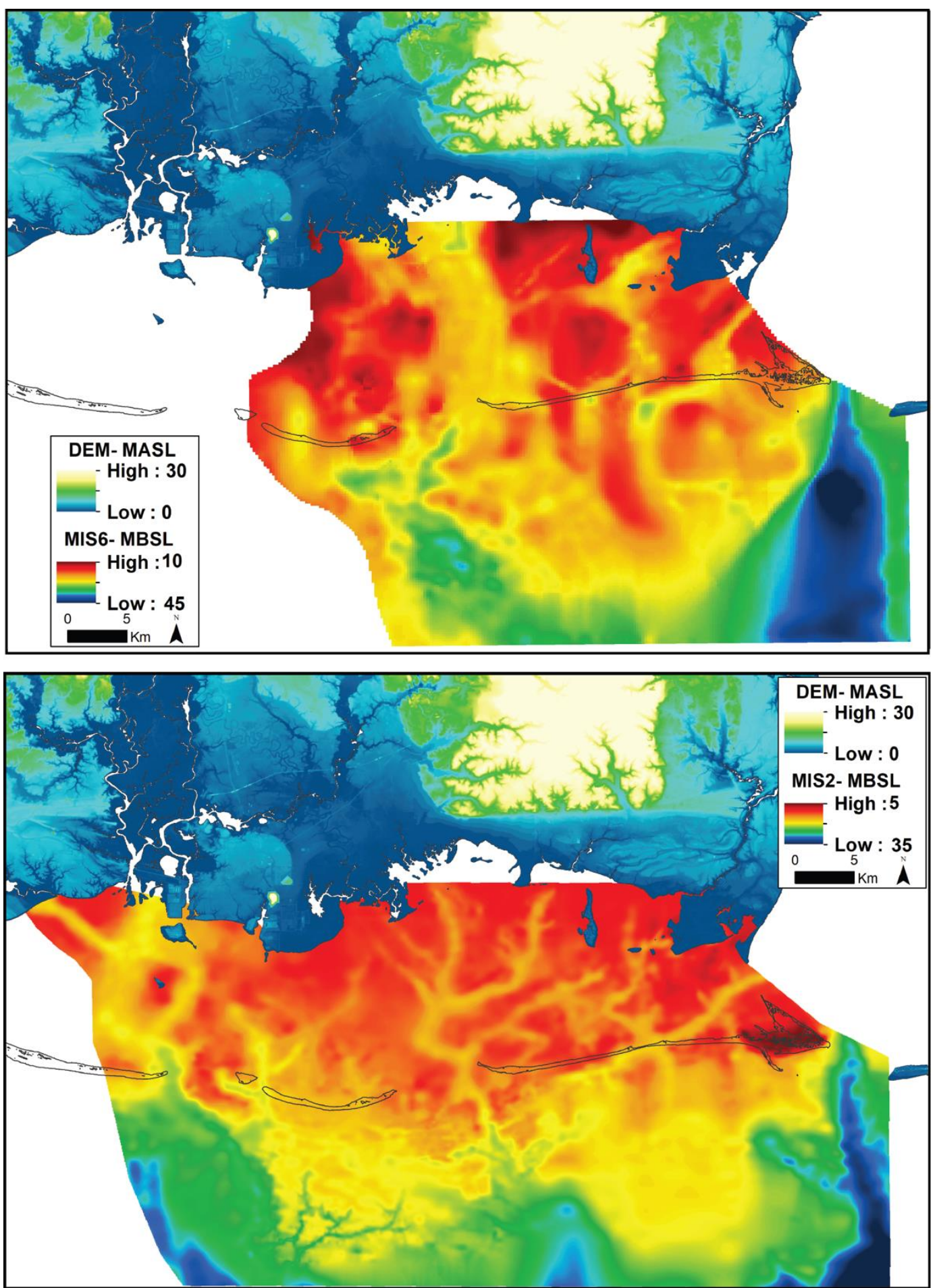

Figure 5 MIS 6 and MIS 2 Surfaces

The MIS 6 and MIS 2 lowstand sequence boundary interpolated surfaces overlain with the 2007 and 2009 composite DEM. Interpolated surface elevation represents meters below sea level (MBSL) and DEM elevations represent meters above sea level (MASL). 


\subsubsection{Sequence Boundary 2: Marine Isotope Stage 2}

Sequence boundary 2 (SB2) is characterized by a low to high amplitude, semi to highly continuous reflector, mapped 3-30 mbsl (0-15m mbsf). Flocks (2015) similarly noted this regional horizon (SB2) truncates SB1, supporting our interpretation that SB2 is indeed younger based on cross cutting relationships (Figure 6 and 7). Radiocarbon samples directly overlying SB2 are Holocene age (Table 2, sample 35, 37, 44). This timetransgressive surface represents the most recent lowstand sequence boundary during the Last Glacial Maximum (MIS 2) (Figure 2 and 5), uncomfortably separating Holocene from Pleistocene units below.

\subsubsection{Unit 1: Pleistocene}

Unit 1 is characterized in sediment cores by indurated, oxidized, mottled yellowish orange, brown gray and greenish grey clays and sandy clays (Figure 8, 9, 10). Paleosol development characterized by oxidized or weathering coloration is common, and represents subaerial exposure processes. Root horizons are fairly common directly overlying this unit indicating this was a vegetated area (Figure 10). This mottled oxidized section is usually found within the upper meter below this contact and not found in all areas, indicating it was either eroded during transgression or located in a subaqueous environment such as a fluvial setting. Cores penetrating unit 1 are characterized by clayey sand to sandy clay to clay deposits with sand and mud filled burrows common near the upper contact. Shell and plant material range from common to absent throughout the entire unit. This unit represents various Pre-Holocene coastal plain deposits from marine through fluvial alluvial facies (Figure 6, 7, 8) (Hummel and Parker 1995, Kramer 1990, Otvos 1981, 1985) that have been correlated to updip formations mapped by Otvos 
(2001) in certain locations (Otvos 1985, 1986). Complete delineation of these formations on the inner shelf is beyond the scope of this study.

Unit 1 has variable reflector configurations and is bound by the incisional Surface 1 and 2 in the lower sequence, and the incisional Surface 2 and the Holocene transgressive ravinement surface in the upper sequence (Figure 6, 7, 8).

\subsubsection{Unit 2: Fluvial Channel Sands Deposits}

Unit 2 consists of tan to white, moderately to poorly sorted fine to gravelly, unidirectional, high angle cross-stratified sands (Parker et al. 1993). Sand sized heavy minerals were also reported. Shells were absent or minimal. Interstate-10 bridge borings (Burns Cooley Dennis Inc., 2016) in the updip section of the Pascagoula River (Figure 1) display correlative 7-16m thick sand deposits matching depths of channel sand packages found in seismic.

Unit 2 is characterized by medium to high amplitude low frequency, chaotic to transparent reflectors with channel fill geometry, which is similar to other work in Florida and Texas (Goff et al. 2014, Thomas and Anderson 1994). This unit was difficult to geophysically image due to its depth in record and mostly homogenous nature. This unit is interpreted as fluvial channel fill sands. Surface 2 and the Holocene transgressive ravinement surface bound the channel fill sands.

\subsubsection{Unit 3: Fluvial Lateral Accretion Deposits}

Unit 3 is sparsely sampled and only represents the very upper section of the deposit. Cores consist of interbedded grayish brown, medium to fine sands and olive-gray muds to tan well sorted, medium to fine sands (Figure 6,8). Where described, shell content was minimal throughout the deposit (Kelso and Flocks 2015). Sand and mud 
filled burrows, as well as wood fragments are common. The few core samples make interpreting between fluvial bar and bayhead delta deposits difficult.

Unit 3 is characterized by low to medium amplitude, medium frequency, steeply dipping oblique or prograding reflectors into the incised valley. In some cases, there is a central mounded feature with opposite dipping reflectors or top lapping horizontal low amplitude reflectors in the upper sections similar to descriptions by other authors (Flocks 2015, Greene et al. 2007, Bartek et al. 2004, Thomas and Anderson 1994). This unit has either basin or channel fill external geometry and is interpreted generally as fluvial lateral accretion deposits. This implies higher sand content type deposits such as fluvial bars or portions of bayhead deltas.

\subsubsection{Unit 4: Marsh Deposits}

Unit 4 consists of humate brown to dark gray peat or rooted zones in a poorly sorted silty sand matrix. Shells are mostly absent, and the sediment is mottled with mud filled burrows (Hummel and Parker 1995). Heavy organic beds are common. This unit was sampled on the flanks within incisional valley features on the inner shelf, and within eastern Mississippi Sound.

Where submerged, unit 4 was $0.2-0.6 \mathrm{~m}$ thick and usually is associated with gas pockets, making it difficult to image in geophysical data (Zaremba et al. 2016). This unit is interpreted as saltmarsh deposits and usually directly overlies Pleistocene deposits, except in backbarrier and bay margin environments (Figure 10). Otvos (1986) reports brackish muddy sediments from the same horizon. This unit was constrained from 7,500-6,300 BP (Table 2, sample 47, 50, 61). 


\subsubsection{Unit 5: Oyster Biostrome}

Unit 5 consists of articulated and unarticulated shell beds in a framework supported matrix of muddy sand and sand. These deposits are not oyster reefs according to Hummel and Parker (1995). This $0.2-0.8 \mathrm{~m}$ thick unit consisted mostly of Crassostrea virginica (10-30 psu) with sparse Ostrea equestris (>30 psu) (Parker 1960). The deposit sampled by Hummel and Parker (1995) within eastern Mississippi Sound was dominated by Crassostrea virginica, which prefer water depths of about $2.5 \mathrm{~m}$ (Parker 1960).

Unit 5 is characterized by high amplitude, mounded to transparent reflectors, with parabolic to chaotic signal masking below similar to Goff et al. (2015) in Corpus Christi Bay, TX. Due to its relatively small spatial extent it was difficult to image but was only found in Pass Aux Heron in eastern Mississippi Sound. This unit was interpreted as an oyster biostrome and formed 3,900 BP (Table 2, sample 57).

\subsubsection{Unit 6: Estuarine Deposits}

Unit 6 consists of light to dark gray-brown, laminated to heavily bioturbated organic silty clay and muddy sands. Some silty sand and sandy silt layers, lenticular bedding, sand/mud pockets and burrows are also found (Greene et al. 2007, Hummel and Parker 1995, Flocks 2015, McBride et al. 1991) (Figure 9, 10). Articulated mollusk and gastropod shells, as well as shell and wood fragments, are common (Figure 7). This layer can be highly mottled. This $0.5-7 \mathrm{~m}$ thick unit was found largely in Mississippi Sound within incisional features identified in geophysical data.

Unit 6 is characterized by low to high amplitude, high frequency, wavy to parallel reflectors but can also be transparent. This unit has sheet drape, basin or channel fill, onlapping external geometry, similar to other studies in the area (Flocks 2015, Greene et 
al. 2007). This unit was interpreted as estuarine deposits of ranging salinities, encompassing the enclosed bay, upper bay, open bay and open bay margin facies of Parker (1960) based on mollusk assemblages. Estuarine deposition started 9,800 BP (Table 2, sample 44), reflecting differences in early valley flooding versus recent deposition within Mississippi Sound (Figure 11).

\subsubsection{Unit 7: Tidal Inlet Fill or Barrier Spit}

Unit 7 consists of grayish brown muddy sand grading to massive fine shelly sand with rare flaser beds. The unit fines upwards to lenticular-bedded clay and muddy fine sand with rare shell fragments and bedded organics, similar to authors in LA, NC/SC, TX (Miner et al. 2009, Moslow and Tye 1985, Simms et al. 2006). This 1.5-3m thick, unit was mostly found landward of Petit Bois Island.

Unit 7 is characterized by low to medium amplitude, laminated to transparent reflectors in the upper section with fill or laterally prograding accreting geometry and a chaotic base (Miner et al. 2009). This unit was interpreted to be tidal inlet fill and first dated to $~ 7,200 \mathrm{BP}$ (Table 2, sample 28). Inlet fill and spit accretion is also evident from modern deposits from historical charts (Morton 2008, Buster and Morton. 2011).

\subsubsection{Unit 8: Flood Tidal Delta Deposits}

Unit 8 consists of brownish gray, laminated to massive silty to very fine-fine sand with some faint multi directional cross stratification and dipping beds (McBride et al. 1991, Siringan and Anderson 1993). There were abundant shell fragment layers and moderate sand filled borrows. This $1-4 \mathrm{~m}$ thick unit was mostly found in the modern tidal inlets (Main Pass (Mobile Bay), Petit Bois Pass, Horn Island Pass) landward of Petit Bois and Horn Islands (Figure 1). Unit 8 is occasionally preserved offshore within valleys. 
Unit 8 is characterized by low to medium amplitude, medium frequency, low to medium angled, landward dipping, oblique reflectors. In a strike view the reflectors are horizontally laminated in a lobate geometry. These reflectors downlap underlying surfaces and show a slight erosive base. This unit has basin fill or lobate external geometry (Miner et al. 2009, Siringan and Anderson 1993), interpreted as flood tidal delta deposits. It was constrained to $\sim 1850 \mathrm{CE}$ to modern from historical charts (Buster and Morton 2011) and radiocarbon dates from landward of Petit Bois at 2,300 BP (Table 1, sample 9) and Dauphin at 4,000 BP (Table 1, sample 16) (Figure 9, 10).

\subsubsection{Unit 9: Ebb Tidal Delta Deposits (Modern)}

Unit 9 is similar to unit 8 except it is coarser grained and displays more sand dominated dark gray, well sorted fine to medium, cross stratified to massive sands with abundant, large shell fragments (McBride et al. 1991, Miner et al. 2009, Siringan and Anderson 1993). Sand filled burrows are more prevalent at distal ends of the ebb tidal delta.

Unit 9 is characterized by seaward dipping reflectors organized in a lobate geometry (Miner et al. 2009, Siringan and Anderson 1993). This unit is interpreted as ebb tidal delta deposits and was only found seaward of modern passes and was not recognized in paleoenvironments. Time-series historical charts show evidence of wave modification, tidal inlet lateral migration, and tidal inlet throat avulsions (Morton 2008, Buster and Morton 2011, Byrnes et al. 2013).

\subsubsection{Unit 10: Shoreface Deposits}

Unit 10 consists of light to dark gray and tan poorly sorted, sometimes interbedded sands and muds, and muddy sands with sand and clay lenticular beds. The 
upper section has higher sand content with few mud beds and Donax sp. shells. It becomes more mud dominated with few, thin sand beds down section (Figure 8, 9, 10) similar to the description of Rodriguez (2001) along the Texas coast. There is an increase in mottling, likely due to bioturbation in the seaward distal sections. This unit also contains wood and shell fragments; clay rip up clasts, peat balls, and sand and mud filled burrows (Rodriguez et al. 2001). This $0.5-3 \mathrm{~m}$ thick deposit was found flanking the modern islands.

Unit 10 is characterized by low to medium amplitude, medium frequency semilaminated or transparent reflectors (Rodriguez et al. 2001). It has sheet drape or wedge external geometry. Due to its relatively thin nature it is sometimes difficult to distinguish from the seafloor in geophysical data. This unit is interpreted as the upper and lower shoreface and has been constrained geologically to 7,000 to 1,800 BP (Table 1, sample 1, 2,3). Shoreface deposits are also present in modern environments.

\subsubsection{Unit 11: Barrier and Backbarrier Deposits}

Two sub-units characterize unit 11 . Sub-unit 1 consists of light gray to white massive to planar laminated and cross-laminated bedding of fine to medium quartz sand. This sub-unit also has shell hash layers, detrital organics, peat rip-up clasts, wood fragments, roots traces and sand and mud filled burrows (Otvos 1981, Moslow and Tye 1985). These 3-14m thick sands comprise the islands. Sub-unit 1 is interpreted as barrier lithosome sands consisting of dune and shore facies.

Sub-unit 2 consists of light gray, massive to horizontal and slightly dipping planar laminated muddy sands, with some interbedded sands and muds. This $0.5-2 \mathrm{~m}$ thick, subunit has many fining upward sequences and is found on the backside of the barrier 
islands. Medium amplitude and frequency, semi-parallel, massive and chaotic reflectors with a lobate or sheet external geometry characterize the subaqueous portion of sub-unit 2. Sub-unit 2 is interpreted as backbarrier overwash deposits.

\subsubsection{Unit 12: Marine Surficial (MAFLA) Sand Sheet}

Unit 12 is composed of tan, massive to crudely planar laminated, fine muddy, shelly quartz sand with some sand and mud filled burrows. It has a sharp basal lag (McBride et al. 1991, Parker et al. 1993).

This $0.5-1.5 \mathrm{~m}$ thick unit is characterized by low to medium amplitude, low frequency, parallel to sub parallel reflectors with a sheet like external geometry (Figure 7, 8) similar to that described in Flocks (2015). Due to the thickness and homogeneity, it is difficult to image in geophysical data. This unit is found throughout the inner shelf from mid Petit Bois eastward and is interpreted as the Mississippi Alabama Florida (MAFLA) sand sheet, which reflects modern shelf current reworking.

\subsubsection{Unit 13: Sand Ridge Deposit}

Unit 13 consists of tan to dark gray, massive to planar laminated, medium to coarse sand, with echinoid spines and a graded shell uppermost section and base (Flocks 2015, McBride et al. 1991, Parker et al. 1993, Goff et al. 2014). This 1-6m thick unit, is found offshore of Petit Bois, Petit Bois Pass, and seaward of Main Pass (Figure 1, 6).

Unit 13 is characterized by low to medium amplitude, low frequency, parallel to sub parallel reflectors, with a slightly erosional basal reflector (Flocks 2015, Goff et al. 2014). This unit has a shore oblique ridge external geometry (Figure 6). Due to homogeneity, it is difficult to image internal structure. Unit 13 is interpreted as modern 
shore oblique attached and detached sand ridges of varying sizes, reworked by modern shelf currents.

\subsubsection{Unit 14: Inner Shelf Mud Deposit}

Unit 14 is composed of greenish- gray bioturbated clean to sandy muds with lower organic content (Thomas and Anderson 1994, Otvos 1981). Marine fossil assemblages and stratigraphic relationships are needed to identify this deposit (Otvos 1981). In seismic it is characterized by wavy or horizontal parallel reflectors or is acoustically transparent, which makes it is indistinguishable from estuarine deposits based on seismic alone (Figure 6) (Thomas and Anderson 1994). This 0.1-2m thick unit is interpreted as inner shelf marine muds.

\subsubsection{Unconformity 1: Transgressive Surface (TS)}

This erosional boundary represents the unconformable separation of lowstand deposits such as fluvial channel fill or alluvium from estuarine or marine deposits above (Figure $6,7,8)$. It is a regionally continuous surface that usually becomes amalgamated with ravinement surfaces in interfluve areas. It is interpreted as the transgressive surface (TS) (Cattaneo and Steel 2003).

\subsubsection{Unconformity 2: Wave Ravinement Surface (wRs)}

This erosional boundary represents the separation of estuarine to alluvial sediments and marine sediments (Figure 6, 7,8). It is usually a high amplitude continuous reflector and consists of a coarse shell lag layer in most cases except in paleovalleys. It may be amalgamated with the transgressive or tidal ravinement surface. This surface is interpreted as the wave ravinement surface (wRs) (Cattaneo and Steel 2003), similar to the shoreface ravinement surface in other studies (Rodriguez et al. 
2001). Its depth varies within the study site. Bathymetric estimates for the toe of the shoreface are estimated from a break in slope at about 9-10mbsl. This is confirmed through seismic and core data showing a wave ravinement surface between 7-10mbsl represented by thick shell hash, marine muds or shelly inner shelf sand sheet deposits onlapping muddy shoreface sands or exposed Pre-Holocene deposits. It defines the seaward extent of the active barrier system for this study.

\subsubsection{Unconformity 3: Tidal Ravinement Surface (tRs)}

This erosional boundary represents the separation of alluvial and estuarine deposits below and tidal deposits above (Figure 6, 7, 8). It was found in paleovalleys, the inner shelf and underlying Dauphin and Petit Bois Island. It occurred below the wave ravinement surface in some cases and may be amalgamated with the transgressive surface, such as in the backbarrier. It is usually represented by a coarser grained shell lag layer with an erosional base and is associated with tidal deposits. This is interpreted as the tidal ravinement surface (tRs) (Cattaneo and Steel 2003).

\subsubsection{Unconformity 4: Bay Ravinement Surface (bRs)}

This erosional boundary represents the separation of alluvial and tidal deposits from estuarine deposits. It is constrained to within Mississippi Sound, eroding Pleistocene unit interfluvial locations and occasionally within fluvial valleys. This boundary is interpreted as the bay ravinement surface (bRs) (Cattaneo and Steel 2003) and represents lower energy wave base erosion relative to open Gulf conditions. This surface can be very difficult to identify in MS Sound if the surface is truncating other estuarine deposits (Greene et al. 2007). 

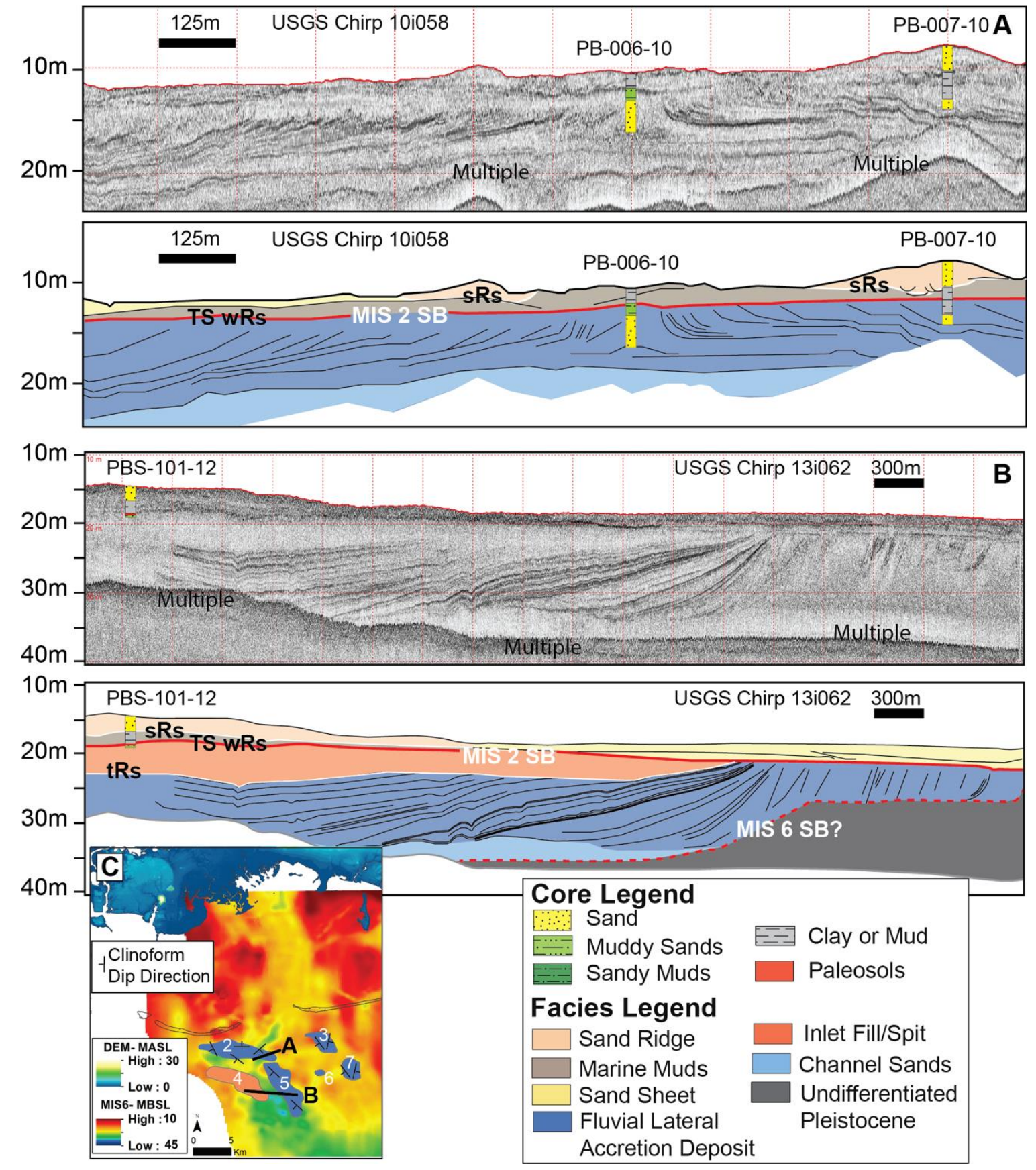

Figure 6 Truncation of MIS 6 Valley Fill

Uninterpreted and interpreted SB-512i chirp profiles (A and B). The MIS 6 and MIS 2 sequence boundaries are shown in red. Ravinement surfaces shown in white unless amalgamated with sequence boundaries. Areal extents of truncated MIS 6 deposits (C) overlain with MIS 6 surface and modern DEM (NOAA 2007, 2009). Clinoform strike and dip estimates are represented by geologic indicators (grey) inferred from 2D seismic at varying orientations. Figure location found in figure 1 and 6C. 


\subsubsection{Unconformity 5: Sand Ridge Ravinement Surface (sRs)}

This erosional boundary represents the separation of sand ridge deposits from highly variable underlying deposits. This surface was interpreted as the sand ridge ravinement surface (sRs) and represents the basal scour erosion from large-scale bedform migration (Goff et al. 2014).

\subsection{Antecedent Topography and Paleofluvial systems}

\subsubsection{Sequence Boundary 1: MIS 6 Surface}

\subsubsection{Fowl and La Batre Systems}

The Fowl River valley is a $0.5-1 \mathrm{~km}$ wide and $20-25 \mathrm{mbsl}$, seaward bifurcating system with a series of smaller tributary drainages (Figure 5). These features are mapped below the shoreface of western Dauphin Island and likely connect with the Pascagoula/Escatawpa valley to the southwest although data density offshore of Dauphin Island is relatively low. A small, narrow, 20-25 mbsl, bifurcation of the Western Mobile Valley system incises Pass Aux Herons (Greene et al. 2007, Hummel and Parker 1995). Mapping of these valley systems further offshore Dauphin Island is difficult due to spacing resolution of available archive data. Paleohighs separating fluvial incisions were also mapped similar to Greene et al. (2007). There is a thin, likely alluvial, depositional package ( 2-5m) separating sequence boundary 1 and 2 on the interfluves (MIS 6 and MIS 2).

Valley fill reported by Greene et al. (2007) consists of sequential fluvial/ alluvial deposits with overlying bayhead delta deposits in the Fowl system and only bayhead delta fill in the La Batre system. The La Batre bayhead delta valley fill is older than MIS 2 interpreted based on two radiocarbon-dead (>48,000 BP) wood samples within this 
deposit (Greene et al. 2007). Rindsberg (1992) reports detailed fossil assemblages that could point towards an ancient delta lobe in the area but it occurs below the MIS 2 unconformity (Hummel and Parker 1995) that is believed to be the same MIS 6 feature.

\subsubsection{Escatawpa/Pascagoula}

The Pascagoula/Escatawpa River valley is a 5-10km wide and 25-38 mbsl seaward widening terraced system (Figure 5a). Similar to the Fowl and La Batre systems, surrounding smaller drainages flow into the main valley trunk, and valley shoulders rise to within a few meters of sequence boundary 2 on the interfluves. It flows under eastern Petit Bois, which is the "MIS 2" valley mapped by Flocks (2015) and the shallower terrace is the valley mapped by Twichell et al. (2011) offshore of Petit Bois Pass. When comparing the geomorphology of the sequence boundary 1 gridded surface with Lidar, valley width and orientation agree (Figure 5). The Lidar shows a bifurcation of valleys to the modern Pascagoula location and to the east towards Grand Bay (Figure 5). This location coincides with the Citronelle fault scarp orientation (Figure 3) (Otvos 1985). The valley fill is capped with Pleistocene Prairie Formation (MIS 5 deposits) alluvium, with Pliocene Citronelle deposits bounding this valley to the northeast (Figure 3).

The valley fill of the Pascagoula/Escatawpa system shows transparent to chaotic reflectors confined to the base of the valley (Figure 6) not directly sampled offshore. However, updip I-10 bridge (Figure 1) borings spanning this valley show a correlative 9m thick sand deposit at similar elevations (Burns Cooley Dennis Inc., 2016). In roughly 15-16m water depth, several, variable size sandy deposits were mapped within the valley. These consisted of oppositely dipping reflectors, which radiate in all directions from a central mounded feature. These steeply dipping reflectors become more gradual from the 
center. Only a few cores penetrate the upper section of this deposit and show sands and interbedded sands and muds near the mounds, with more mud dominated deposits in distal sections (Figure 6). Based on these characteristics and the fact they are only found within this large valley, these features are interpreted as fluvial lateral accretion deposits. Seaward of Petit Bois, these fluvial/deltaic features are truncated by an erosional boundary interpreted as the Holocene transgressive ravinement surface (TS) (Figure 6). Within Mississippi Sound, thinly laminated- semi wavy parallel reflectors overly fluvial channel sands and are interpreted as estuarine or alluvial deposits. Greene et al. (2007) did not report a large MIS 6 Pascagoula/Escatawpa system. This could be a result of the limited western extent of their survey and the shallow geophysical data penetration of the 216s chirp compared to the boomer seismic data used in this study (Figure 4).

\subsubsection{Sequence Boundary 2: MIS 2 Surface}

\subsubsection{MIS 2 Surface Gradient}

Five mainland to backbarrier transects within MS Sound and five inner shelf transects measure the average slope of the MIS 2 surface within the study site. Transect locations focused on slope of interfluve areas. The average slope of the MIS 2 surface within MS Sound is $-0.069 \mathrm{~m} / \mathrm{km}$, ranging from $-0.256 \mathrm{~m} / \mathrm{km}$ (western study site) to $0.009 \mathrm{~m} / \mathrm{km}$ (eastern study site). This reflects the southwest dip of the surface. The average slope of the inner shelf is $-0.734 \mathrm{~m} / \mathrm{km}$, ranging from $-0.867 \mathrm{~m} / \mathrm{km}$ (western study site) to $-0.663 \mathrm{~m} / \mathrm{km}$ within the study site (eastern study site). The order of magnitude difference in MIS 2 interfluve slope between Mississippi Sound and the Gulf of Mexico reflect a significant antecedent feature underlying the modern location of the MSAL barrier chain. 


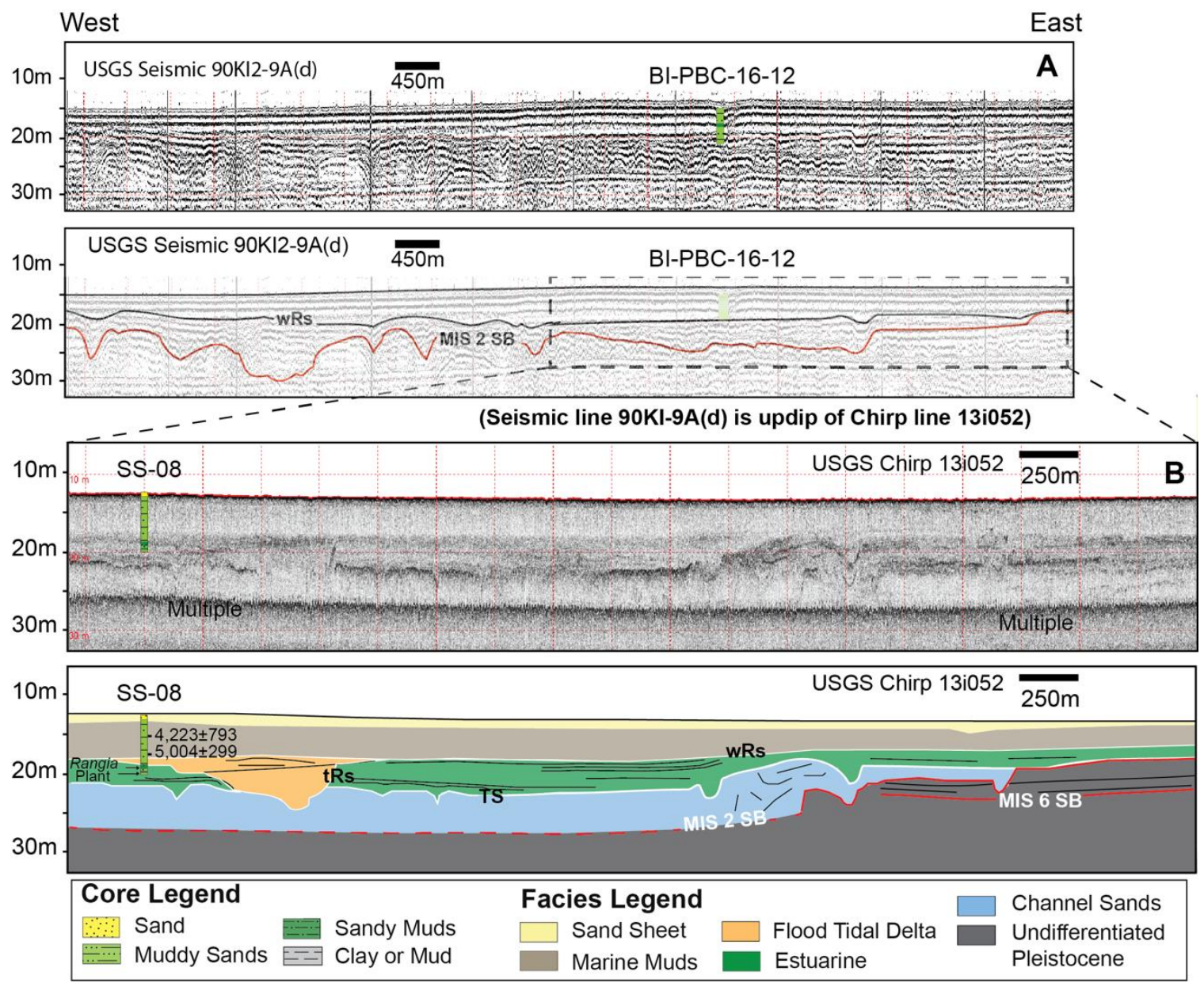

Figure 7 MIS 2 Pascagoula Valley Fill

Uninterpreted and interpreted seismic (A) and SB512i chirp lines (B). Seismic line 90KI2-9A(d) shows a much more complete strike orientation of the Pascagoula valley seaward of Horn Island Pass. Detailed interpretation of ravinement surfaces (white) are shown in Chirp line 13i052, which is slightly down dip from the seismic line. Line locations found in figure 1.

\subsubsection{Fowl, La Batre, Escatawpa Systems}

The Fowl incisional system is less then $1 \mathrm{~km}$ wide and incises to a depth of 10-14

mbsl (Figure 5b). The La Batre system is even smaller. Greene et al. (2007) reports a series of narrow tributaries incising to 16-17 mbsl but were not mapped since they were beyond the resolution of the survey. These incisional features are filled solely with clay and clayey sand, transparent to wavy parallel reflectors that are interpreted as estuarine deposits. Cores from Hummel and Parker (1995) describe thin peat horizons directly overlying the subaerially exposed MIS 2 sequence boundary (surface 2). A series of 
anastomosing dendritic tributary/distributary channel networks was mapped in this study using additional geophysical data. The tributaries continue under the western portion of Dauphin Island (Fig. 5). Estuarine deposition filled these deeper tributary systems. A gently sloping peat facies within eastern Mississippi sound was constrained to roughly 7,500-6,200 BP (Table 2, sample 47, 48, 50, 61), which is consistent with sea level elevations during this period (Milliken et al. 2008).

The Escatawpa drainage system shows small tributary channels about $0.2-0.8 \mathrm{~km}$ wide that incise to a depth of 4-10 mbsl within Mississippi Sound. Mullenex (2013) shows a series of roughly $150 \mathrm{~m}$ wide, roughly $2-6 \mathrm{~m}$ deep " $\mathrm{v}$ " shaped MIS 2 channel incisions that extend into this study's data coverage. The Escatawpa lacks a major confining MIS 2 valley (Kramer 1990, Mullenex 2013). The incisional geometries and geomorphologies match updip comparisons in Lidar (Figure 5). Kramer (1990) cores show relatively thin $1-2 \mathrm{~m}$ thick, Holocene fluvial/deltaic sands interpreted as distributary mouth bars overlain by deltaic/ marsh facies proximally located to Grand Bay.

\subsubsection{Pascagoula System}

The Pascagoula system is $1-5 \mathrm{~km}$ wide and incises to $15-25 \mathrm{mbsl}$, flowing south through Horn Island and Horn Island Pass. Several complex interfluve drainage tributaries flow into the main valley trunk, but are difficult to map due to the geophysical data spacing resolution in Mississippi Sound. An interfluve seaward of Petit Bois Island 


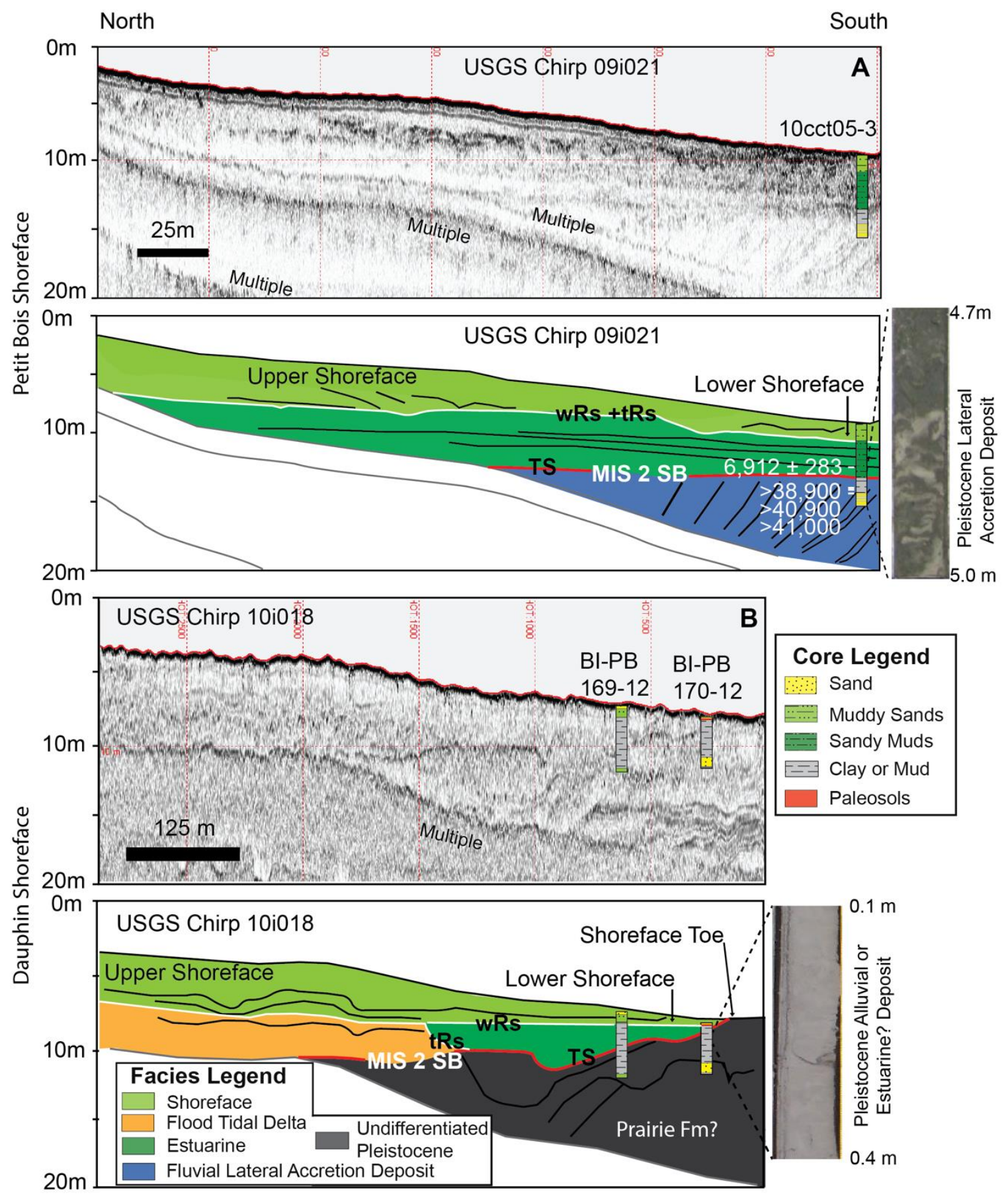

Figure 8 Petit Bois and Dauphin Shoreface

Uninterpreted and interpreted shoreface chirp profiles from Petit Bois (09i021, top) and Dauphin Island (10i018, bottom) with sediment cores displaying a thin sandy shoreface and underlying Pleistocene deposits (Kelso and Flocks 2015, USACE 2014). Pleistocene fluvial lateral accretion deposits also shown in figure $6 \mathrm{~A}$ and 10 . Shoreface toe shown at $\sim 7 \mathrm{mbsl}$ by exposed Pleistocene deposits (B). Location found in figure 1.

displays a series of $\sim 200 \mathrm{~m}$ wide, 25 mbsl southwestward draining tributaries coalescing at the main Pascagoula valley (Figure 5). Again, this is consistent with drainage networks 
in terrestrial updip sections, especially in the Citronelle and Prairie Formations. A larger tributary drainage underlies the western portion of Petit Bois. Cores from the interfluve areas and overlying portions of the MIS 6 valley show a shallow subaerially exposed surface (relative to the seafloor). Flocks (2015) shows the eastern valley (interpreted as the older, MIS 6 sequence in this study) reflector is truncated to the west by the MIS 2 Pascagoula valley (Figure 5).

Chirp data show fluvial channel sands at the base (6m), estuarine, and tidal deposits grading into $8 \mathrm{~m}$ thick marine deposits, separated by a series of ravinement surfaces (Figure 7). Incorporating seismic data shows the rough base of the fluvial deposits, although no cores penetrate this layer offshore (Figure 7). Estuarine and marine muds are differentiated by the description of plant fragments and Rangia sp. shells at the base and an overlying layer with dated marine mollusks. Estuarine fill seaward of Horn Island Pass has been constrained to 9,800-5,100 BP (Otvos 1986) (Table 2, sample 43, 44).

In Mississippi Sound, the Pascagoula valley base is difficult to image. R/V Erda 92-4 cruise seismic data seaward of the modern Pascagoula rarely penetrates beyond 2025 mbsl. Two I-10 bridge borings (Burns Cooley Dennis Inc., 2016) show 7-16m thick, medium to coarse sands at $8-12 \mathrm{~m}$ below the surface overlying stiff or dense clays, which is interpreted as fluvial channel sands at the base of the Pascagoula valley (MIS 2). Up to $5 \mathrm{~m}$ of thinly laminated, wavy parallel reflectors drape Mississippi Sound. This sandy mud and mud deposit is exposed at the seafloor and is interpreted as estuarine deposition. No tidal or fluvial deltaic valley fill was identified within the Mississippi Sound.

\subsection{Sand Volume Calculations}


Seven relatively large sandy fluvial or deltaic deposits were identified based on core and geophysical data within the study area, and all but one are located on the inner shelf (Figure 6). Based on a ravinement surface identified in geophysical data that truncates sandy deposits preserved on the shelf, sand scoured during the Holocene transgression could have supplied up to $306.7 \times 10^{6} \mathrm{~m}^{3}$ (Table 4). This is a conservative estimate based on the lower range of wave ravinement $(7-10 \mathrm{mbsl})$ calculated in this study. This volume is limited to the sandy valley fill deposits and not the entire shelf. This represents about $60 \%$ of the sand volume of the active Petit Bois and Dauphin barrier platform and modern sand ridges and sand sheet (Table 4).

Table 4 Sand Source and Sink Volumes

\begin{tabular}{cccc}
$\begin{array}{c}\text { Modern Sink } \\
\text { Unit }\end{array}$ & Volume $\left(\mathrm{x} 10^{6} \mathrm{~m}^{3}\right)$ & Ancient Source Unit & $\begin{array}{c}\text { Reworked } \\
\text { volume }\left(\mathrm{x} 10^{6}\right. \\
\left.\mathrm{m}^{3}\right)(7 \mathrm{~m} \mathrm{rav})\end{array}$ \\
\hline $\begin{array}{c}\text { Sand Ridges } \\
\text { Inter Ridge area } \\
\begin{array}{c}\text { Active Barrier } \\
\text { System }\end{array}\end{array}$ & $8.7^{*}$ & Fluvial Deposit (2) & 82.5 \\
& $9.4^{*}$ & Fluvial Deposit (3) & 33.0 \\
& 455.0 & Reworked FTD (4) & 65.4 \\
& & Fluvial Deposit (5) & 96.5 \\
& & Fluvial Deposit (6) & 3.5 \\
Total Sand & & Fluvial Deposit (7) & 25.8 \\
Volume & 473.1 & Total Possible & 306.7 \\
\hline
\end{tabular}

Volumes for Holocene sink and reworked MIS 6 source units. * Notes volumes adopted from Flocks (2015), sand content estimated from cores. Reworked volumes calculated by multiplying preserved area and the depth of ravinement similar to Weight et al. 2011. Offshore source units correspond to figure $6 \mathrm{c}$. 

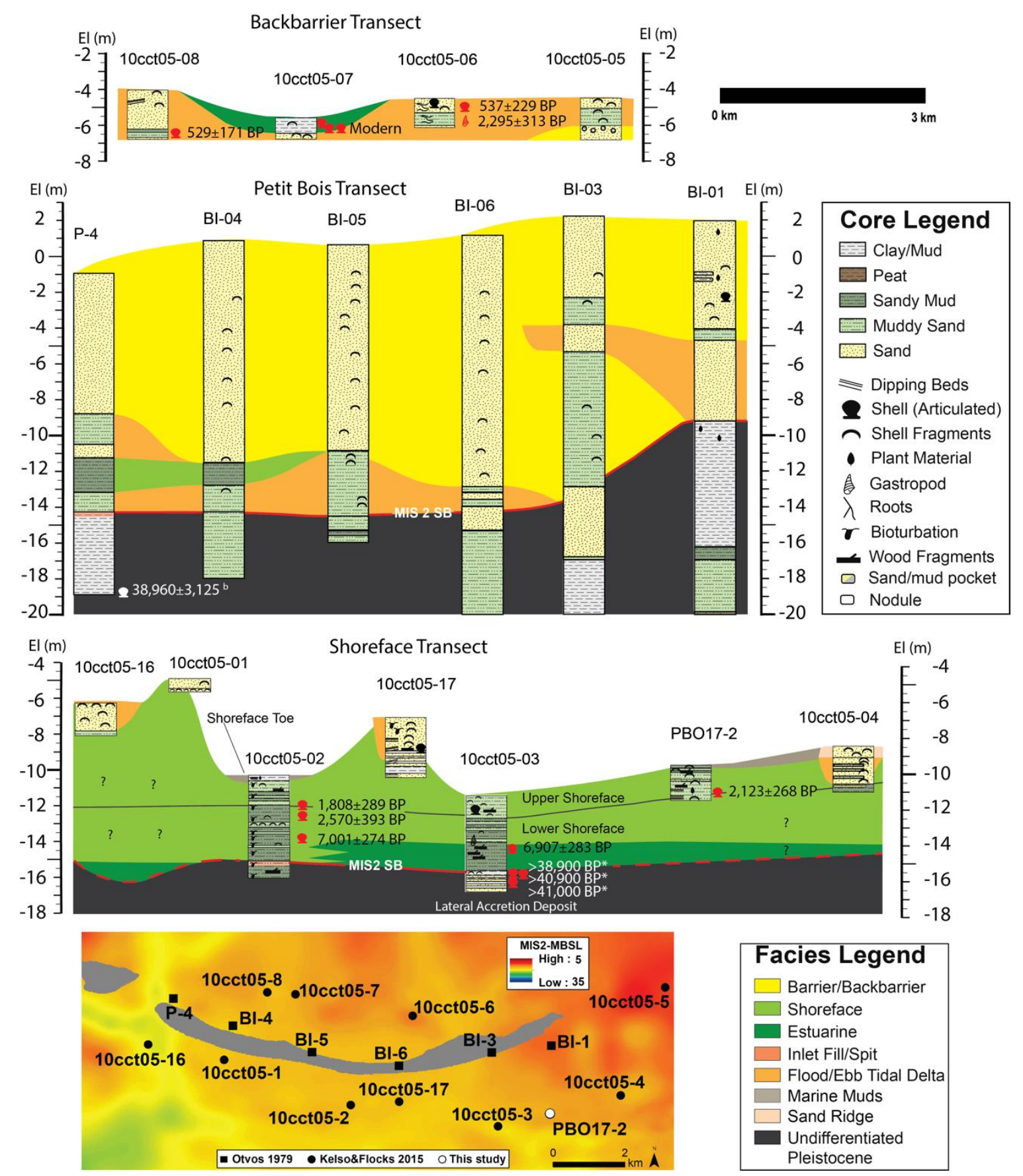

Figure 9 Petit Bois Shore Parallel Stratigraphic Cross Sections

Backbarrier, barrier, and shoreface cross sections, synthesizing marine vibracores (Kelso and Flocks 2015), Otvos (1979) rotary drill cores and dive assisted percussion core (this study). Core locations overlie MIS 2 sequence boundary surface. Core 10cct05-3 is shown in corresponding shoreface chirp line in figure (8). Facies interpretations aided by previous microfossil salinity data (Otvos 1979, 1985). See table 1 and 2 for radiocarbon details. b notes previously reported contaminated sample, * notes radiocarbon dead sample. Shoreface toe shown in core 10cct05-02 at 10mbsl, onlapping marine muds to shoreface sand. 


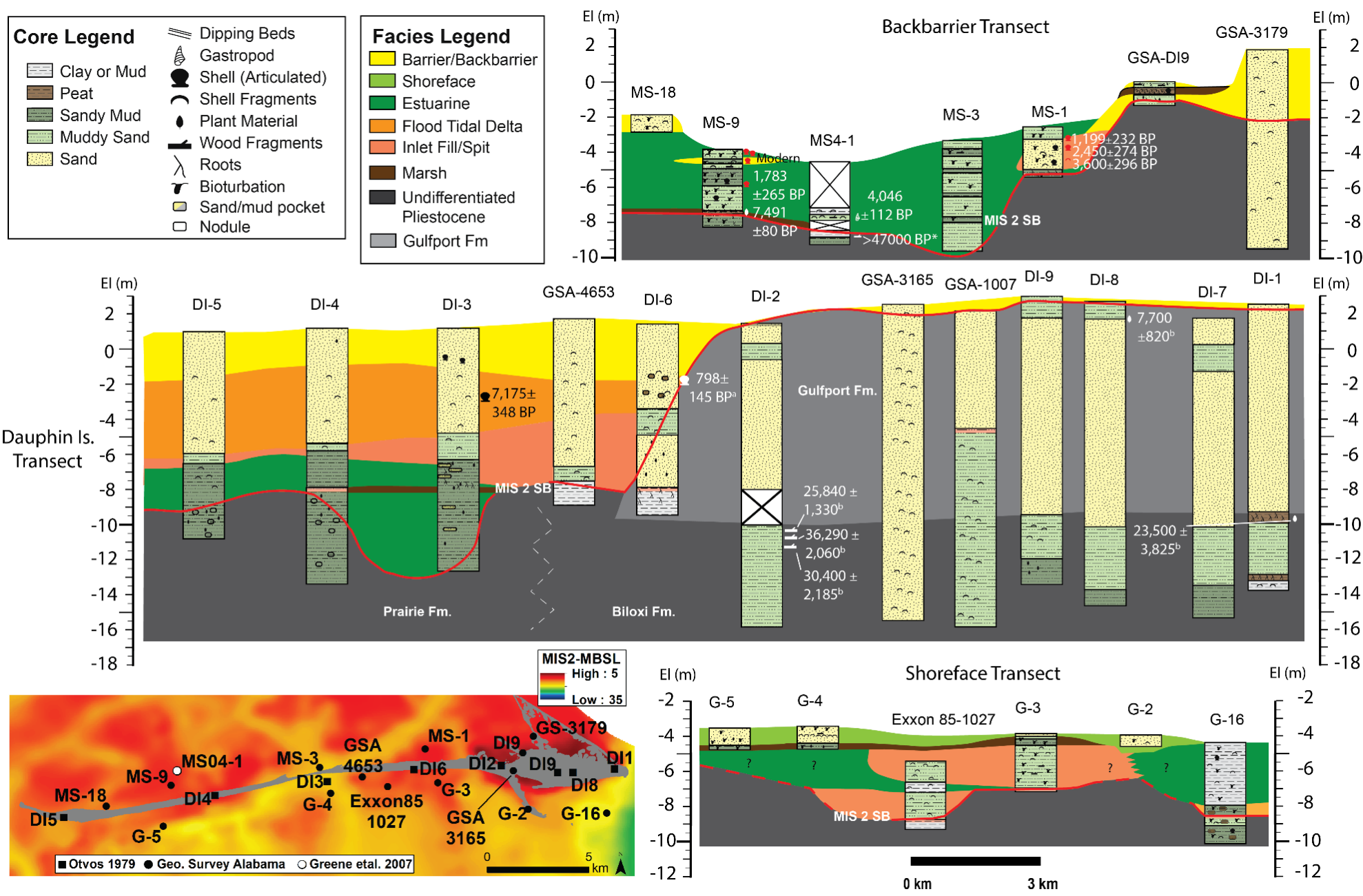

Figure 10 Dauphin Shore Parallel Stratigraphic Cross Sections

Backbarrier, barrier, and shoreface cross sections, synthesizing Otvos (1979) and Greene et al. (2007) rotary drill cores and vibracores (Hummel and Parker 1995, Hummel 1999, and references within). The core locations overlie the MIS2 sequence boundary surface. Facies interpretations were aided from previous microfossil salinity information (Otvos 1979). See table 1 and 2 for radiocarbon details. b Notes previously reported contaminated sample, * notes radiocarbon dead sample. 


\section{5-DISCUSSION}

\subsection{Pre-Holocene Antecedent Geology and Topography Influences}

The different valley geometry, fill architecture and therefore sediment supply during transgression between the MIS 2 and MIS 6 valleys reflect different duration and magnitudes of sea level forcing mechanisms. The MIS 6 lowstand had more extreme base level fall for longer periods relative to MIS 2 (Shackelton 1987, 2000). In Texas and Louisiana, the MIS 6 valleys are much larger and have higher sediment loads compared to MIS 2 (Anderson et al. 2004). During the MIS 6 lowstand the large Pascagoula/Escatawpa river valley flowed through the Grand Bay area, depositing many sandy valley fill units that later would supply sand to the modern barrier island chain through transgressive ravinement (Figure 5 and 6). The terraced valley geometry increases preservation potential during the MIS 5 transgression (Rodriguez et al. 2008). Collectively, an estimated $183.4 \times 10^{6} \mathrm{~m}^{3}$ of sand is contained within MIS 6 valley fill (bayhead delta and transgressive fill described in Flocks (2015)). The MIS 6 valley likely had a high sediment discharge based on the thick, acoustically transparent, channel sand package at the base of the valley just seaward of Grand Bay.

Sometime during or prior to the MIS 2 lowstand, the main Pascagoula River valley trunk avulsed to its western, modern outlet location (Figures 3 and 5). Smaller MIS 2 tributaries show fluvial reoccupation of MIS 6 valleys (Green et al. 2007). The MIS 2 surface dips to the southwest similar to previous studies (Kramer 1990, Greene et al. 2007, Ludwick 1964, Otvos 1985) likely due to sediment loading from the Mississippi River deltas. The modern day undammed Pascagoula shows large point bars updip, directly eroding the sandy Pliocene Citronelle Formation. It likely had a similar 
meandering channel belt and point bar configuration down dip within the study site during the MIS 2 lowstand. Seaward of the islands, the thin preserved channel sands imply it was likely reworked during transgression (Figure 7). The shallow, wide valley geometry has little preservation potential during transgression (Rodriguez et al. 2008). Based on fill and valley geometries, the shallow eastern portion of Mississippi Sound is characterized by smaller, mud filled-anastomosing tributary drainages (Ndjorford et al. 2005, Greene et al. 2007) with little to no fluvial channel sands at the base. These tributary drainages likely supplied very little sand during the transgression.

Modern barrier chain geomorphology shows correlation to the MIS 2 or antecedent topographic surface, similar to other studies (Zaremba et al. 2016, Mallinson et al. 2010, Rodriguez et al. 2001, Browder and McNich 2006, Riggs et al. 1995). The MIS 2 surface shows a significant break in slope between the modern Mississippi Sound and the inner shelf. This gradient change aligns with the MIS 5e barrier trend between Dauphin and Fort Morgan, AL, and the modern day MSAL barrier chain. A break in slope was originally suggested to anchor the modern barriers but was never regionally mapped (McBride et al. 1991 and Otvos 1981) until now. The combination of decelerating sea level rise rates (Figure 2) intersecting the MIS 5e barrier section of Dauphin Island (Figure 3) and the MIS 2 gradient change (Figure 5) is responsible for the modern position of the MSAL chain.

MIS 2 valley location correlates with modern day points of weakness in the MSAL barrier chain (Figure 5), such as tidal inlets or areas of frequent storm breaching and overwash. Petit Bois Pass and Horn Island Pass overlie the large main MIS 2 Escatawpa tributary and Pascagoula valley, respectively (Figure 5). Katrina Cut in the 
western spit of Dauphin Island overlies a smaller tributary channel. The storm breach separating East and West Ship Island, MS is also located at a paleodrainage location (Twichell et al. 2013). This is due to the wave and tidal erosion resistance variability between dense clay rich MIS 2 interfluves and the less dense younger valley fill (Figure 8). Valleys can be sites of local subsidence as muddy fill compresses (Figure 10). Preexisting semi consolidated, sandy deposits likely offer little erosion variability compared to Holocene deposits.

\subsection{Sediment Sourced through Transgressive Ravinement}

Truncation of MIS 6 sand rich deposits was likely a major sediment source for the modern barrier island chain through transgressive ravinement (Table 4; Figure 6). Studies from glacial New England coastlines (Schwab et al. 2013) and other coastal plain settings in the Gulf of Mexico (Weight et al. 2011, Rodriguez et al. 2001, Wallace et al. 2010, Anderson et al. 2016 and references within) demonstrate the significance of stratigraphic framework and onshore transport of sands to modern barriers. Conservatively estimating the sand sourced from reworking of lowstand deposits could have supplied about $60 \%$ of the modern sinks (barrier platform, sand ridges and sand sheet). Once the sand was reworked, some of this volume was likely transported down drift to other islands in the chain. However, this reworked sand volume estimate is from only one valley. The change in MIS 2 gradient at the modern barrier chain is likely a result of wave ravinement variability. Bay ravinement is much less efficient due to reduced wave climate compared to the open Gulf (Cattaneo and Steel 2003). Paleochannels preserved in Mississippi Sound are completely truncated in the shoreface due to wave and tidal scour offshore of Horn Island Gal et al. (in prep). Entire sea level cycles are not preserved only $30 \mathrm{~km}$ east 
of the study site (Gonzalez et al. 2017). Offshore Fort Morgan, a preserved, radiocarbon dead (<40ka), late Pleistocene cypress forest underlies a thin post transgressive marine sediment package, representing a major unconformity (Gonzalez et al. 2017). The examples of transgressive ravinement presented in this study and others (Gal et al. in prep, Gonzalez et al. 2017) illustrates the importance of onshore sand transport to the modern MSAL barrier systems.

\subsection{Holocene Transgressive Evolutionary Model}

\subsubsection{0ka-9ka Transgressing Shoals}

According to the Milliken et al. (2008), sea level was about $16 \mathrm{~m}$ below modern around 9ka (Figure 11) with RSLR rates around 9mm/yr (Anderson et al. 2016). Bayhead delta deposition started around 9.7ka in northern Mobile Bay (Greene et al. 2007). Mobile Bay transitioned from upland or marsh to an estuary between 9.2ka and 9ka (Greene et al. 2007, Metcalfe and Rodriguez 2003). Around this time, the Pascagoula valley transitioned from fluvial to estuarine dominated processes within the southern study site. This is demonstrated by a $9.8 \mathrm{ka}$ piece of wood from the base of a brackish sandy mud in core SS-11 offshore of Horn Island Pass (Otvos 1986) (Table 2, sample 44). Due to its wide, shallow natured geometry and low preservation of transgressive fill succession, it likely became a wave-dominated estuary. Fluvial sand transport to the coast ceased, and transgressive ravinement processes took over (Anderson et al., 2014), likely acting as a critical sediment source to the MSAL chain later on. Some form of physical barrier such as an ephemeral island or marine shoal seaward of this position (Figure 11) created the estuarine, brackish salinities reported by Otvos (1986). 
Water depths inside the southern portion of the Pascagoula Valley were up to $8 \mathrm{~m}$, tidal and wave processes likely eroded basal fluvial sands. Water depths overlying the older MIS 6 sandy deposits ranged from 2-8m suggesting they were actively being reworked during this time. This is also inferred from the prominent ravinement surface (Figure 6) constrained to older then 7ka (Figure 8; Table 1, sample 4). Milliken et al. (2008) reports a sea level stillstand during $9.5 \mathrm{ka}$, which would enhance the efficiency of shoreface ravinement. Bathymetric highs such as interfluve and valley shoulder areas likely focused wave energy, reworking deposits and transporting them landward.

Alongshore transport of sand was likely inefficient due to the immature, irregular shaped coastline.

\subsubsection{8-7ka Transgressing Shoals}

RSLR rates were rapid between 8-7ka with many flooding events (average of 4$5 \mathrm{~mm} / \mathrm{yr}$, and as high as 6-7mm/yr during flooding events; Anderson et al. 2016, Milliken et al. 2008). Paleo sea levels were about $13 \mathrm{~m}$ below present rising to $7 \mathrm{~m}$ below present by $7 \mathrm{ka}$ (Figure 11). Estuarine deposition dominates the Pascagoula valley within Mississippi Sound constrained by peat and wood samples from brackish sediments described by Otvos (1979) (Table 2, sample 33, 35, 37). Estuarine conditions now exist north of Petit Bois in a flooded tributary (Table 2, sample 31). Ephemeral islands or shoals likely mirrored antecedent topographic geometries. Transgressive shoals were near the modern position of Horn Island based on 8ka washover/tidal deposits (Gal et al. in prep). The majority of sand was still being sourced from reworking of the inner shelf.

By $7 \mathrm{ka}$ RSLR has slowed to about 4mm/yr. The Gulfport Formation section of east Dauphin of the MIS 2 surface acted as a nucleation point for shoal belt deposition as 
marine flooding reached this area around $7 \mathrm{ka}$. An ephemeral island or migrating marine shoal similar to the description of Otvos (1981), was located about $2 \mathrm{~km}$ seaward of modern Petit Bois Island. This is inferred from similar age and elevation estuarine and sandy nearshore deposits (Figure 11; Table 1, sample 3, 4). This transgressive sand body was unable to stabilize during rapid sea level rise (Figure 6, Table 1, sample 3). Microfossil salinity data within Mississippi Sound shows upward and landward freshening of mud or muddy sand units resulting from a combination of river discharge and the migrating shoal belt (Otvos 1985, 1986, 1981).

A coastal marsh formed atop antecedent highs in the eastern section of Mississippi Sound (Figure 11, Table 2, sample 47, 48, 49, 50). Although neither Hummel and Parker (1995) or Greene et al. (2007) discussed whether this was salt or freshwater peat, its elevation and age correspond to within $1 \mathrm{~m}$ of sea level at this time, well within tidal range. Microfossil salinity data from this area suggest brackish environments (Otvos 1985). Otvos (1985) shows the presence of calcareous nearshore marine and intertidal foraminifera found in the deposits proximal to the peats. Therefore, we interpret these as salt marsh deposits. The peats directly overlie the subaerial MIS 2 surface and Hummel (1999) report them underlying sections of the Dauphin Island shoreface (Figure 10). This indicates the early transgressive phase of these landward migrating marine shoals. Deeper drainages in this area likely became tidally influenced estuarine tributaries (Table 2, sample 28). No peats were found in the central or western portion of Mississippi Sound indicating the flooded antecedent topography was deep enough for estuarine deposition or larger waves and more efficient bay ravinement eroded the deposit completely. 

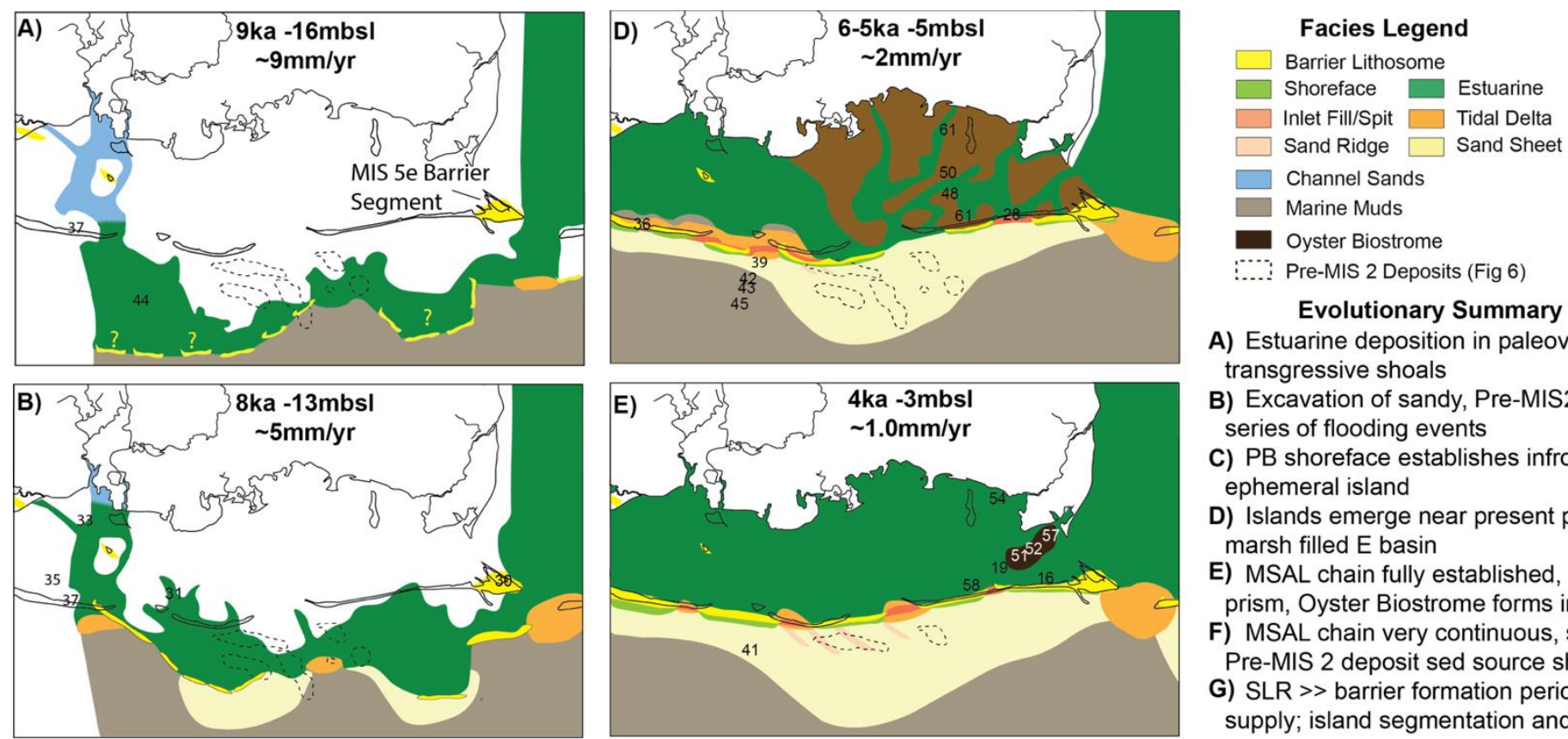

\section{Evolutionary Summary}

A) Estuarine deposition in paleovalleys,

transgressive shoals

B) Excavation of sandy, Pre-MIS2 deposits,

series of flooding events

C) PB shoreface establishes infront of

ephemeral island

D) Islands emerge near present positions,

marsh filled $\mathrm{E}$ basin

E) MSAL chain fully established, large tidal

prism, Oyster Biostrome forms in E basin

F) MSAL chain very continuous, stormy period

Pre-MIS 2 deposit sed source shut off

G) SLR >> barrier formation period (D), lower sed supply; island segmentation and land loss
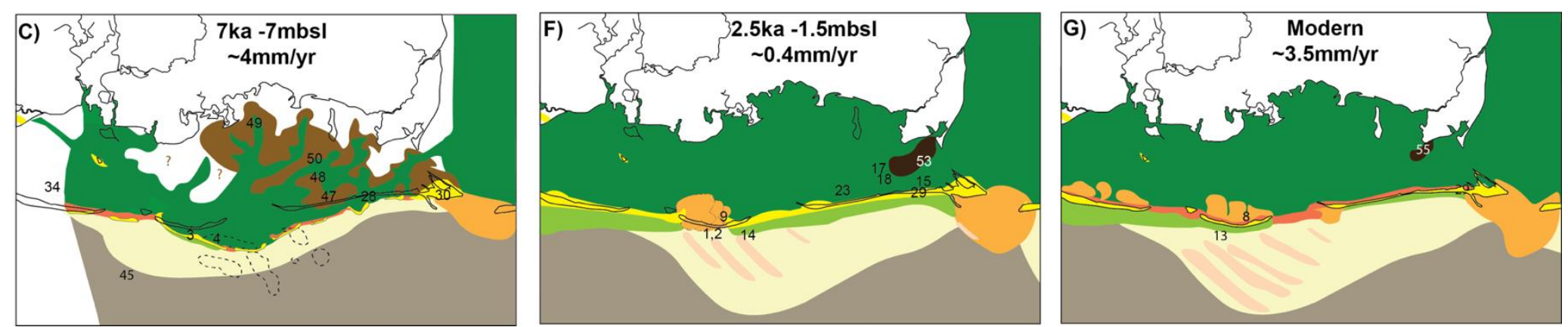

Figure 11 Holocene Evolutionary Model

Paleogeographic maps showing the flooding of MIS2 surface based on seismic and core interpretations at various Holocene time intervals. Paleo sea level was inferred from the Milliken et al. (2008) non-linear, sea level curve (Figure 2). Black lines represent modern shorelines; white areas are land. Numbers represent radiocarbon samples cross referenced to tables 1 and 2 to aid in interpretation of depositional environments. Dotted lines represent pre-MIS2 sandy fluvial/deltaic deposits being reworked assuming $7 \mathrm{~m}$ of wave ravinement; this sand source is eventually shut off as sea levels rise. 


\subsubsection{6-5ka: Island Formation and Stabilization}

Sea level reached the modern mainland shoreline at $6 \mathrm{ka}$, increasing the tidal prism of Mississippi Sound. Between 5.7-5.0 ka RSLR rates were about 2mm/yr (Milliken et al. 2008), allowing the migrating marine shoals to reach a stabilization threshold (Otvos and Carter 2004). The Gulfport section of eastern Dauphin Island, abundant sediment supply and the minor sea level rise rates allowed the islands to establish and aggrade vertically near their modern positions (Otvos 1979,1981, 2018) (Figure 11). Otvos (1970, 1979, 1981) described a marine sandy mud unit underlying the Horn Island platform (Otvos 1981). This is interpreted as distal flood tidal deposits as the shoals or ephemeral islands continually breached and migrated landward. The newly flooded antecedent topography of Mississippi Sound due to the MIS 2 gradient change from $-0.734 \mathrm{~m} / \mathrm{km}$ to -0.069 $\mathrm{m} / \mathrm{km}$ (Figure $5 \mathrm{~b}$ ) significantly increased accommodation space. The slower sea level rise likely increased wave ravinement efficiency. The uniform paleo water depths suggest the island chain was becoming more linear and mature.

Fort Morgan Peninsula began to prograde and aggrade at 5.3ka (Rodriguez et al. 2006) constraining the Mobile inlet throat and establishing the Mobile ebb tidal delta. The connection between updrift sand sources suggests sediment supply to the MSAL was both onshore and alongshore transport.

\subsubsection{4-2.5ka: Island Progradation}

RSLR rates slowed from about $1.4 \mathrm{~mm} / \mathrm{yr}$ to $0.4 \mathrm{~mm} / \mathrm{yr}$ (Milliken et al. 2008) and sea levels were about 3 meters below modern. Tidal and wave scour begin bypassing deeper inner shelf MIS 6 sandy deposits (Figure 6 and 11). The abundant sediment supply from reworked deposits $\left(306.7 \times 10^{6} \mathrm{~m}^{3}\right)($ Table 4$)$ and alongshore sources 
(Mobile ebb tidal delta and eroding Pleistocene headland in Florida) likely caused rapid westward lateral progradation of the MSAL chain around 4ka (Figure 11) (Otvos 1979). The MSAL barrier chain extended as far as New Orleans at one time (Otvos 1981, 2018). The emergence of oyster biostromes about 3.8ka, landward of Dauphin Island, indicates a fairly continuous barrier island chain with sufficient tidal circulation to Mississippi Sound. The barrier trend was held at its modern position due to the large accommodation space of the flooded antecedent topography of Mississippi Sound and the alongshore currents anchored by the MIS 5e section of Dauphin Island. It is possible due to inlet migration and abundant sand that the shoreface oblique sand rides began to form according to the McBride and Moslow (1991) translating ebb tidal delta model.

As the islands laterally accrete, tidal inlets can become anchored in incised valleys (Simms et al. 2006, Siringan and Anderson 1993) such as the deep tributary underlying western Petit Bois (Figure 5, 9, 11). Amalgamated, laterally offset, flood tidal delta and attendant tidal channel deposits are present landward of western Petit Bois (Figure $1,5 \mathrm{~b}, 9,11)$. These are characterized by vertically aggraded cut and fill patterns in seismic profiles constrained to $2.3 \mathrm{ka}$ (Table 1 , sample 9). The bathymetric extent of these large flood tidal deltas can be seen in modern bathymetry (Figure 1; NOAA 2007, 2009). Similar relict tidal deltas exist between East and West Ship, which is spatially correlated to paleo tributaries (Twichell et al. 2013).

As pre-existing sand sources on the shelf were exhausted, barrier stabilization and progradation was maintained through lateral inlet migration (Simms et al. 2006, Otvos 1970) to keep pace with RSLR. The islands overlie a thick (3-16m) medium-fine sandy platform (Otvos and Giardino 2004), which could have provided ample sands through 
tidal ravinement as islands prograded westward. The sea level still stand allowed Petit Bois to prograde seaward about $2 \mathrm{~km}$ around 1.8-2.5ka (Table 1, sample 1, 2, 14) (Figure 9, 11). Gal et al. (in prep) and Misellis et al. (2014) show a progradational trend slightly earlier further west in the MSAL chain (2.2-3.8ka Horn Island, 2.5-4ka Cat Island). This implies earlier evidence of Petit Bois progradation was likely reworked or reflects variability in antecedent topography/sediment supply. Studies from coastal Mississippi indicate that 2,200-1,900 BP was a relatively intense stormy period (Bregy et al. 2018). It is possible this increased storm wave action reworked earlier prograded shoreface deposits at Petit Bois. Theoretically inlets were opened through storm breaching, some were maintained like the one landward of western Petit Bois but generally the islands remained stable through this time. It is likely that modern geomorphology such as storm breaches and inlets correspond to MIS 2 valleys (Mallinson et la. 2010, Zaremba et al. 2016, Siringan and Anderson 1993, Twichell et al. 2013).

\subsubsection{CE- Modern: Island Degradation}

The sea level stillstand (Milliken et al. 2008) ended fairly abruptly, when RSLR rates increased to $\sim 2 \mathrm{~mm} / \mathrm{yr}$ (Gerlach et al. 2017). This is about the same rate in which the islands stabilized 5.7-5.0 ka (Otvos and Giardino 2004). The majority of sand is transported westward from updrift sources. The combination of increased RSLR and reduced sediment supply led to island thinning and breaching. The 1752 CE D'anville chart shows Petit Bois and Dauphin joined as one island which was later separated by a storm in the late 1700's (Otvos and Giordano 2004). Lateral inlet migration throughout historic times (150 years) (Buster and Morton. 2011) has completely reworked most of Dauphin and Petit Bois through tidal ravinement. Both the modern Petit Bois Pass and 
the Katrina Cut on Dauphin Island correlate to MIS 2 tributary channels (Figure 5 and 10). Lower elevation antecedent topography correlates with erosional hotspots or points of weakness during storms (Browder and McNich 2006, Twichell et al. 2013). Islands are severely declining in areal extent, thinning from the Gulf and Sound sides (Morton 2008). Modern sediment budgets indicate mixed results as some clearly show defined littoral cells with little exchange between islands (Cipriani and Stone 2001, Morton 2008) and others show sediment transport generally decreases downdrift (Byrnes et al. 2013). Petit Bois and Dauphin show relatively sand starved features. The lack of ridge and swale topography on the low elevation western spit results from a combination of anthropogenic influences that have altered sediment dynamics and frequent overwash. Dauphin Island's shoreface is sand starved (Figure 8 and 10).

Modern RSLR rates at Dauphin Island are $3.61 \mathrm{~mm} / \mathrm{yr} \pm 0.59 \mathrm{~mm} / \mathrm{yr}$ (NOAA 2018: Station 8735180), and with very little sand supplied by proximal wave ravinement, the western of Dauphin spit cannot keep up with modern SLR (Figure 6). The shoreface shows very little sand above the stiff, Pleistocene muddy alluvial deposits (Prairie Formation) (Otvos and Carter 2004) (Figure 8 and 10). Other sediment starved beaches show increased overwash during the current, relatively low storminess period illustrating the systems' sensitivity to accelerated RSLR rates (Rodriguez et al. 2018 (Onslow Beach, NC), Odezulu et al. 2018 (Follets Island, TX)). The relatively deep, unconfined Mississippi Sound has greater accommodation space than sediment supply can fill at the current rate of SLR, pushing these islands past stabilization thresholds. This illustrates the significance of changes in sediment supply compared to sea level rise rates and the antecedent topography underlying the barrier islands. 


\section{6-CONCLUSION}

This study provides a high spatial and temporal resolution data set constraining the Late Quaternary chronostragraphic evolution of the eastern MSAL barrier chain and Mississippi Sound in response to sea level fluctuations, changes in sediment supply, geomorphic changes, and antecedent topography. The underlying geologic framework directly controls the Holocene evolution of the MSAL barriers. During early transgression, wave ravinement excavated up to $300 \times 10^{6} \mathrm{~m}^{3}$ that was available for incorporation into the barrier system. However, fluvial transport of sand to the study area shut off in the Early Holocene. Estuarine conditions were present in paleovalleys seaward of the modern MSAL chain 9ka, suggesting the presence of some form of seaward physical salinity barrier. However, up until about $6 \mathrm{ka}$, sea level rise rates $(<2 \mathrm{~mm} / \mathrm{yr})$ were too rapid for barrier islands to establish, resulting in landward migrating transgressive

ephemeral islands or marine shoals. Sand was supplied mostly from reworked inner shelf sources at this time. As sea level rise rates decreased $(>2 \mathrm{~mm} / \mathrm{yr})$, the balance of sediment supply and accommodation space favored island stabilization as shoal belts vertically aggraded near their present positions about 5.7-5.0ka. The islands sit atop a break in the MIS 2 slope and are anchored by the MIS 5e Gulfport Formation barrier section at Dauphin Island. The MIS 2 surface gradient change is believed to be a result of wave ravinement. Shortly after $4 \mathrm{ka}$, relict sand deposits on the inner shelf were bypassed by transgressive processes. Alongshore and onshore sand supply allowed for island progradation both laterally and seaward during the sea level still stand from about 4ka$1750 \mathrm{CE}$ (1-0.4 mm/yr). From $1750 \mathrm{CE}$ to modern day, the islands have experienced segmentation, thinning and frequent overwash due to reduced sediment supply and 
accelerating sea level rise. Modern tide gauges report $3.61 \mathrm{~mm} / \mathrm{yr} \pm 0.59 \mathrm{~mm} / \mathrm{yr}$ of RSLR in the area, which is higher than the rate at which the islands formed. Mississippi Sound has large accommodation space, and the islands experienced reduced sediment supply compared with geologic times. As future storminess is predicted to intensify and become more frequent coupled with accelerated sea level rise, the islands may return to a transgressive shoal belt similar to their history 6-7ka. This study provides geologic evidence of coastal system geomorphic threshold crossings related to sea level rise and sediment supply and antecedent topography, which can be used to help predict barrier response to future sea level rise and storm scenarios. 


\section{REFERENCES}

Abdulah, K.C., Anderson, J.B., Snow, J.N., Holdford-Jack, L., 2004. The Late Quaternary Brazos and Colorado deltas, offshore Texas-Their evolution and the factors that controlled their deposition, in Anderson, J.B., and Fillon, R.H., (Eds.), Late Quaternary Stratigraphic Evolution of the Northern Gulf of Mexico: SEPM, Special Publication 79, 237-271.

Allen, G.P., Possamentier, H.W., 1993. Sequence stratigraphy and facies model of an incised valley fill: Gironde Estuary, France. Journal of Sedimentary Petrology 63, 378-391.

Anderson, J.B., Wallace, D.J., Simms, A.R., Rodriguez, A.B., Weight, R.W., Taha, Z.P., 2016. Recycling Sediments Between Source and Sink During a Eustatic Cycle. Case Study of Late Quaternary Northwestern Gulf of Mexico Basin. EarthScience Reviews 153, 111-138.

Anderson, J.B., Wallace, D.J., Simms, A.R., Rodriguez, A.B., Milliken, K.T., 2014. Variable response of coastal environments of the northwestern Gulf of Mexico to sea-level rise and climate change: implications for future change. Marine Geology 352, 348-366.

Anderson, J.B., Rodriguez, A., Abdulah, K.C., Fillon, R.H., Banfield, L.A., McKeown, H.A., Wellner, J.S., 2004. Late Quaternary stratigraphic evolution of the northern Gulf of Mexico: a synthesis. In: Anderson, J.B., Fillon, R.H. (Eds.), Late Quaternary Stratigraphic Evolution of the Northern Gulf of Mexico Margin. Society for Sedimentary Geology, Special Publication 79, 1-23.

Bartek, L.R., Cabote, B.S., Young, T., Schroeder, W., 2004, Sequence stratigraphy of a continental margin subjected to low-energy and low-sediment supply environmental boundary conditions: late Pleistocene-Holocene deposition offshore Alabama, U.S.A., in Anderson, J.B., Fillon, R.H. (Eds.), Late Quaternary Stratigraphic Evolution of the Northern Gulf of Mexico Margin. Society for Sedimentary Geology, Special Publication 79, 85-109.

Belknap, D.F., Kraft, J.C., 1985. Influence of antecedent geology on stratigraphic preservation potential and evolution of Delaware's barrier systems. Marine Geology 63, 235-262.

Boone, P.A., 1973. Depositional systems of the Alabama, Mississippi, and western Florida coastal zone. Transactions - Gulf Coast Association of Geological Societies 23, 266-277.

Bosse, S.T., Flocks, J.G., Forde, A.S., 2017a. Digitized analog boomer seismic-reflection data collected during U.S. Geological Survey cruises Erda 90-1_HC, Erda 901_PBP, and Erda 91-3 in Mississippi Sound, June 1990, and September 199.: U.S. Geological Survey Data Series 1047, https://doi.org/10.3133/ds1047.

Bosse, S.T., Flocks, J.G., Forde, A.S., 2017b. Archive of digitized analog boomer seismic reflection data collected during U.S. Geological Survey cruise Acadiana 87-2 in the northern Gulf of Mexico, June 1987. U.S. Geological Survey data release, https://doi.org/10.5066/F7F47MC2.

Bregy, J.C., Wallace, D.J., Minzoni, R.T., Cruz, V.J., 2018. 2500-year paleotempestological record of intense storms for the northern Gulf of Mexico, 
United States. Marine Geology 396, 26-42

http://dx.doi.org/10.1016/j.margeo.2017.09.009.

Browder, A., McNich, J., 2006. Linking framework geology and nearshore morphology: Correlation of paleo-channels with shore-oblique sandbars and gravel outcrops. Marine Geology 2312, 141-162.

Brown, G.F., Foster, V.M., Adams, R.W., Reed, E.W., Padgett, H.D., 1944. Geology and groundwater resources of the coastal area in Mississippi. Mississippi State Geological Survey Bulletin 60, 220.

Buster, N.A., Morton, R.A., 2011. Historical bathymetry and bathymetric change in the Mississippi-Alabama coastal region, 1847-2009. U.S. Geological Survey Scientific Investigations Map 3154, 1 sheet, 13-p. pamphlet. 〈http://pubs.usgs. gov/sim/3154/> .

Burns Cooley Dennis Inc. 2016. I-10 Eastbound Bridge over Pascagoula River Project No: NHPP-0010-01(160)/107215-101000 Jackson County, Mississippi (Core descriptions).

Byrnes, M.R., Rosati, J.D., Griffee, S.F., Berlinghoff, J.L., 2013. Historical Sediment Transport Pathways and Quantities for Determining an Operational Sediment Budget: Mississippi Sound Barrier Islands. Journal of Coastal Research 63, 166183.

Cattaneo A., Steel R.J., 2003. Transgressive deposits: a review of their variability. Earth Science Reviews 62, 187-228.

Chesapeake Technologies, 2018. SonarWiz 6 software https://chesapeaketech.com/ (Last accessed July 21, 2018)

Cipriani, L.E., Stone, G.W., 2001. Net Longshore Sediment Transport and Textural Changes in Beach Sediments along the Southwest Alabama and Mississippi Barrier Islands, USA. Journal of Coastal Research 17, 443-458.

Dalrymple, R.W., Boyd, R., Zaitlin, B.A., 1994. History of research, types and internal organization of incised-valley systems: introduction to volume, in Dalrymple, R.W., Boyd, R., and Zaitlin, B.A., eds., Incised-Valley Systems: Origin and Sedimentary Sequences: SEPM, Special Publication 51, 1-10.

Dolan, G., Wallace, D.J., 2012. Policy and Management Hazards along the Upper Texas Coast. Ocean \& Coastal Management, 59, 77-82.

Eisemann, E.R., Wallace, D.J., Buijsman, M.C., Pierce, T., 2018. Response of a vulnerable barrier island to storm impacts: LiDAR-data-inferred morphodynamic changes on Ship Island, Mississippi, USA. Geomorphology 313, 58-71.

Eleuterius, C.K., Beaugez, S.L., 1980. Mississippi Sound, a hydrographic and climatic atlas: Mississippi-Alabama Sea Grant Consortium MASGP-79-009, Blossom Printing, Inc. Ocean Springs, MS. 135.

Emanuel, K., 2005. Increasing destructiveness of tropical cyclones over the past 30 years. Nature 436, 686-688.

Emiliani, C., 1955. Pleistocene temperatures. Journal of Geology 63, 538-578.

Fillon, R.H., Kohl, B., H.H., Roberts, 2004. Late Quaternary deposition and paleobathymetry at the shelf-slope transition, ancestral Mobile River delta complex, northeastern Gulf of Mexico, in Anderson, J.B., and Fillon, R.H., (Eds.), 
Late Quaternary Stratigraphic Evolution of the Northern Gulf of Mexico: SEPM, Special Publication 79, 111-141.

FitzGerald, D.M., Kulp, M.A., Penland, S., Flocks, J., and Kindinger, J., 2004.

Morphologic and stratigraphic evolution of muddy ebb-tidal deltas along a

subsiding coast: Barataria Bay, Mississippi River Delta: Sedimentology 51, 11571178. doi:10.1111/j.1365-3091.2004.00663.x.

Flocks, J.G., Kindinger, J.L., Kelso, K.W., Bernier, J., DeWitt, N., FitzHarris, M., 2014, Near-surface stratigraphy and morphology, Mississippi inner shelf, northern Gulf of Mexico: U.S. Geological Survey Open-File Report 2015-1014, 19 doi:10.3133/ofr20151014.

Flocks, J.G., Kindinger, J.L., Kelso, K.W., 2015. Geologic control on the evolution of the inner shelf morphology offshore of the Mississippi barrier islands, northern Gulf of Mexico, USA, Continental Shelf Research, 101, 59-70, ISSN 0278-4343, http://dx.doi.org/10.1016/i.csr.2015.04.008.

Flocks, J., Miner, M., Twichell, D., Lavoie, D., Kindinger, J., 2009. Evolution and preservation potential of fluvial and transgressive deposits on the Louisiana inner shelf: understanding depositional processes to support coastal management. GeoMarine Letters 29, 359-378.

Forde, A.S., Dadisman, S.V., Flocks, J.G., Wiese, D.S., 2011a, Archive of digital Chirp sub bottom profile data collected during USGS cruises 09CCT03 and 09CCT04, Mississippi and Alabama Gulf Islands, June and July 2009: U.S. Geological Data Series 590, 6 DVDs.

Forde, A.S., Dadisman, S.V., Flocks, J.G., Wiese, D.S., DeWitt, N.T., Pfeiffer, W.R., Kelso, K.W., Thompson, P.R., 2011b, Archive of digital Chirp sub bottom profile data collected during USGS cruises 10CCT01, 10CCT02, and 10CCT03, Mississippi and Alabama Gulf Islands, March and April 2010: U.S. Geological Data Series 611, 11 DVDs.

Forde, A.S., Flocks, J.G., Kindinger, J.L, Bernier, J.C., Kelso, K.W., Wiese, D.S., 2015, Archive of Digital Chirp Sub bottom Profile Data Collected During USGS Cruise 13CCT04 Offshore of Petit Bois Island, Mississippi, August 2013: U.S.

Geological Survey Data Series 924, 3 DVDs, available at https://dx.doi.org/10.3133/ds924.

Gerlach M.J., Engelhart S.E., Kemp, C.A., Moyer, R.P., Smoak, J.M., Bernhardt, E., Cahill, N., 2017. Reconstructing Common Era relative sea-level change on the Gulf Coast of Florida. Marine Geology 390, 254-269 http://dx.doi.org/10.1016/j.margeo.2017.07.001

Greene, D.L., Rodriguez, A.B., Anderson, J.B., 2007. Seaward-branching coastal-plain and piedmont incised-valley systems through multiple sea-level cycles: late Quaternary examples from Mobile Bay and Mississippi Sound, U.S.A. Journal of Sedimentary Research 77, 139-158.

Goff, J., 2014. Seismic and core investigation off Panama City, Florida, reveals sand ridge influence on formation of the shoreface ravinement. Continental Shelf Research 88, 34-46.

Goff, J., Lurgin, L. Gulick, S.P., Thirumulai, K., Okumura, Y., 2015. Oyster reef die-offs in stratigraphic record of Corpus Christi Bay, Texas, possibly caused by drought- 
driven extreme salinity changes. The Holocene 26, 511-519 DOI:

$10.1177 / 0959683615612587$.

Gonzalez, S., Bentley, S. J., Sr., DeLong, K. L., Xu, K., Obelcz J., Truong, J., Harley, G. L., Reese, C. A., Caporaso, A., 2017. Facies reconstruction of a late Pleistocene cypress forest discovered on the northern Gulf of Mexico continental shelf: Gulf Coast Association of Geological Societies Transactions, 67, 133-146.

Hadden, C.S., Cherkinsky A., 2016. Spatiotemporal variability in $\Delta \mathrm{R}$ in the northern Gulf of Mexico, USA. Radiocarbon doi10.1017/RDC 2016.65.

Hadden, C.S., Cherkinsky A., 2017. Carbon reservoir effects in eastern oyster from Apalachicola Bay, USA. Radiocarbon 24, 1-10 doi:10.1017/RDC.2017.45.

Hapke, C.J., Plant, N.G., Henderson, R.E., Schwab, W.C., Nelson, T.R., 2016. Decoupling processes and scales of shoreline morphodynamics. Marine Geology 381, 42-53.

Hayes, M.O., Nairn, R.B., 2004. Natural maintenance of sand ridges and linear shoals on the U.S. Gulf and Atlantic continental shelves and the potential impacts of dredging. Journal of Coastal Research 20, 138-148.

Honeycutt, M.G., Krantz, D.E., 2003. Influence of the geologic framework on spatial variability in long-term shoreline change, Cape Henlopen to Rehoboth Beach, Delaware. Journal of Coastal Research, Special Issue 38, 147-167.

Hummel, R.L., Parker S.J., 1995. Holocene Geologic History of Mississippi Sound, Alabama. Geological Survey of Alabama Circular 185, 1-91.

Hummel, R.L., Smith, E.V., 1996. Geologic resource delineation hydrographic characterization of an offshore sand resource site for use in beach nourishment projects on Dauphin Island, Alabama. Final Report Geological Survey of Alabama Circular 189, 1-131.

Hummel, R.L., 1999. Holocene Geologic Evolution of the west Alabama Inner Shelf, Alabama. Final Report Geological Survey of Alabama Circular, 1-131.

Ivins, E.R., Dokka, R.K., Blom, R.G., 2007. Post-glacial sediment load and subsidence in coastal Louisiana. Geophysical Research Letters 34, 1-5 doi:10.1029/2007GL030003.

Kelso, K.W., Flocks, J.G., 2015. Archive of sediment data from vibracores collected in 2010 offshore of the Mississippi barrier islands: U.S. Geological Survey Data Series 903, http://dx.doi.org/10.3133/ds903.

Kemp, A.C., Horton, B.P., Donnelly, J.P., Mann, M.E., Vermeer, M., Rahmstorf, S., 2011. Climate related sea-level variations over the past two millennia. Proceedings of the National Academy of Sciences 108, 11017-11022. http://dx.doi.org/10.1073/pnas.1015619108.

Kindinger, J.L., 1988, Seismic stratigraphy of the Mississippi-Alabama shelf and upper continental slope: Marine Geology 83, 79-94.

Kindinger, J.L., 1989, Depositional history of the Lagniappe Delta, northern Gulf of Mexico: Geo-Marine Letters 9, 59-66.

Kindinger, J.L., Balson, P.S., Flocks, J.G., 1994, Stratigraphy of the MississippiAlabama shelf and the Mobile River incised-valley system, in Dalrymple, R.W., Boyd, R., and Zaitlin, B.A., eds., Incised-Valley Systems: Origin and Sedimentary Sequences: SEPM, Special Publication 51, 83-95. 
Kramer, K.A., 1990, Late Pleistocene to Holocene geologic evolution of the Grande Batture headland area, Jackson County, Mississippi, Starkville [unpublished M.S. Thesis]: Mississippi State University, Mississippi State, 1-165.

List, J. H., Jaffe, B. E., Sallenger, A. H., JR., Williams, S. J., McBride, R. A., and Penland, S., 1994. Louisiana Barrier Island Erosion Study: Atlas of Seafloor Changes from 1878 to 1989. Reston, Virginia: U.S. Geological Survey and Louisiana State University, Miscellaneous Investigations Series I-2150- A, 1-81.

Ludwick, J.C., 1964. Sediments of the northeastern Gulf of Mexico. In: Miller, R.L., (ed.), Papers in Marine Geology: Shepard Commemorative Volume: MacMillan: New York, 204-238.

Mallinson, D.J., Culver, S.J., Riggs, S.R., Thieler, E.R., Foster, D., Wehmiller, J., Farrell, K.M., Pierson, J., 2010. Regional seismic stratigraphy and controls on the Quaternary evolution of the Cape Hatteras region of the Atlantic passive margin, USA. Marine Geology 268, 16-33.

Mattheus, C.R., Rodriguez, A.B., Greene, D.L., Simms, A.R., Anderson, J.B., 2007. Control of upstream variables on incised-valley dimension. Journal of Sedimentary Research 77, 213-224.

McBride, R.A., Moslow, T.F., 1991. Origin, evolution, and distribution of shoreface sand ridges, Atlantic inner shelf. U.S.A. Marine Geology 97, 57-85.

McBride, R.A., Byrnes, M.R., Penland, S., Pope, D.L., Kindinger, J.L., 1991, Geomorphic history, geologic framework, and hard mineral resources of the Petit Bois Pass area, Mississippi-Alabama: SEPM, Gulf Coast Section, 12th Annual Research Conference, Program, and Abstracts, 116-127.

Metcalf, M.J., Rodriguez, A.B., 2003. Sedimentary facies and Holocene evolution of the Mobile bay-head delta, Alabama: Proceedings, International Conference on Coastal Sediments 2003, 1-8.

Miner, M. D., Kulp, M. A., Fitzgerald, D. M., 2007. Tidal Versus Shoreface Ravinement Surfaces and Tidal Inlet Fill Preservation Potential, Mississippi River Delta Plain, U.S.A. Journal of Coastal Research, SI 50 (Proceedings of the 9th International Coastal Symposium), 805 - 809. Gold Coast, Australia, ISSN 0749.0208

Miner, M.D., Kulp, M.A., FitzGerald, D.M., Georgiou, I.Y., 2009. Hurricane-associated ebb-tidal delta sediment dynamics. Geology 37, 851-854.

Milliken, K.T., Anderson, J.B., Rodriguez, A.B., 2008. A new composite Holocene sealevel curve for the northern Gulf of Mexico. In: Anderson, J.B., Rodriguez, A.B. (Eds.), Response of Upper Gulf Coast Estuaries to Holocene Climate Change and Sea-Level Rise. Geological Society of America, Boulder 1-11.

Misellis, J., Buster, N., Kindinger, J., 2014. Refining the link between the Holocene development of the Mississippi River Delta and the geologic evolution of Cat Island, MS; implications for delta-associated barrier islands. Marine Geology 355, 274-290.

Mitchum, R.M., P.R. Vail, J.B. Sangree, 1977, Seismic stratigraphy and global changes of sea level, Part 6: Stratigraphic interpretation of seismic reflection patterns in depositional sequences. In: Payton, C.E., (Eds.), Seismic Stratigraphy Applications to Hydrocarbon Exploration, Memoir 26, American Association of Petroleum Geologists 117-133 
Morton, R.A., 2008. Historical changes in the Mississippi-Alabama barrier islands and the roles of extreme storms, sea level and human activities. Journal of Coastal Research 24, 1587-1600.

Moslow, T.F., Tye, R.S., 1985. Recognition and characterization of Holocene tidal inlet sequences. Marine Geology 63, 129-151. http://dx.doi.org/10.1016/00253227(85) 90081-7.

Mullenex, A.J., 2013. Spatial Correlation between Framework Geology and Shoreline Morphology in Grand Bay, Mississippi. [unpublished M.S. Thesis]: Mississippi State University, Mississippi State, 1-192.

Newcome, R. Jr., 1967. Ground Water Resources of The Pascagoula River Basin Mississippi and Alabama. United States Geological Survey Report, Water-Supply Paper 1839-K 1-36.

NOAA, 2007. Biloxi Mississippi, 1/3 arc second Coastal Digital Elevation Model https://data.noaa.gov/dataset/dataset/biloxi-mississippi-coastal-digital-elevationmodel (Last accessed June 28, 2018).

NOAA, 2009. Mobile, Alabama 1/3 arc second Coastal Digital Elevation Model https://catalog.data.gov/dataset/mobile-alabama-coastal-digital-elevation-model (Last accessed June 28, 2018).

NOAA, 2018. Sea Level Trends, Dauphin Island, AL Station 8735180. URL. https://tidesandcurrents.noaa.gov/sltrends/sltrends_station.shtml?id=8735180, (Last accessed August 12, 2018).

Nordfjord S., Goff, J.A., Austin J.A., Sommerfield C.K., 2005. Seismic Geomorphology of buried channel systems on the New Jersey outer shelf: assessing past environment conditions. Marine Geology.214, 339-364.

Odezulu C.I., Lorenzo-Trueba J., Wallace D.J., Anderson J.B., 2018. Follets Island: a case of unprecedented change and transition from rollover to subaqueous shoals. In: Moore L.J., Murray A. (Eds.), Barrier dynamics and response to changing climate. Springer, Cham, pp. 147-174.

Otvos E.G., 1979. Barrier island evolution and history of migration, north central Gulf Coast. In: S.P. Leatherman (Editor), Barrier Islands. Academic Press, New York, N.Y., 291-319.

Otvos, E.G., 1981. Barrier island formation through nearshore aggradation — stratigraphic field evidence. Marine Geology 43, 195-243.

Otvos, E.G., 1985. A New Stratigraphic System, Geologic Evolution and Potential Economic Resources in the Mississippi Sound Area, Mississippi-Alabama, 71 p., Open-file Report, the Mississippi Mineral Resources Institute; 1-71 (Mississippi Sound and bay Drill Logs, with Lithology and Fossil content.).

Otvos, E.G., 1986. Stratigraphy and Potential Economic Sand Resources of the Mississippi-Alabama Barrier Island System and Adjacent Offshore Areas, 67 p. Open-file Report, the Mississippi Mineral Resources Institute; 1-67. (Detailed Island Drill Logs, with Lithology and Fossil Content).

Otvos, E.G., 2001. H. Mississippi Coastal: Stratigraphy and Quaternary Evolution in the Northern Gulf Coastal Plain Framework. U.S. Geological Survey Open-file Report 01-415-H. 
Otvos, E.G., Giardino, M.J., 2004. Interlinked barrier chain and delta lobe development, northern Gulf of Mexico. Sedimentary Geology 169, 47-73.

Otvos, E.G., Carter, G.A., 2008. Hurricane degradation-barrier development cycles, northeastern Gulf of Mexico: landform evolution and island chain history. Journal of Coastal Research 24, 463-478.

Otvos, E.G., Carter, G.A., 2013. Regressive and transgressive barrier islands on the North-Central Gulf Coast: Contrasts in evolution, sediment delivery and island vulnerability. Geomorphology 198, 1-19.

Otvos, E.G., 1970. Development and Migration of Barrier Islands, North Gulf of Mexico. Geological Society of America Bulletin 81, 241-246.

Otvos, E.G., 2018. Coastal barriers, northern Gulf-Last Eustatic Cycle; genetic categories and development contrasts. A review. Quaternary Science Reviews 193, 212-243.

Parker, R.H., 1960. Ecology and Distributional Patterns of Macro-Invertebrates, Northern Gulf of Mexico American Association of Petroleum Geologists Special Publications 21, 302-337.

Parker S.J., Davies, D.J., Smith, W.E., 1993. Geological, Economic, Environmental Characterization of Selected Near-Term Leasable Offshore Sand Deposits and Competing Onshore Sources for Beach Nourishment. Geological Survey of Alabama Final Report.

Pendleton, E.A., Barras, J.A., Williams, S.J., Twichell, D.C., 2010. Coastal Vulnerability Assessment of the Northern Gulf of Mexico to Sea-Level Rise and Coastal Change. USGS Report Series 2010-1146, 1-26.

Pilarczyk, J.E., Barber, D.C., 2015. Mollusca. In: Shennan, I., Long, A.J., and Horton, B.P. (Eds.), Handbook of Sea-Level Research. John Wiley \& Sons, 258-267.

Raff, J.L., Shawler, J.L., Ciarletta, D.J., Hein, E.A., Lorenzo-Trueba, J., Hein, C.J., 2018. Insights into barrier-island stability derived from transgressive/regressive state changes of Parramore Island, Virginia. Marine Geology 403, 1-19.

Reimer, P.J., Reimer, R.W. (2001) A marine reservoir correction database and on-line interface. Radiocarbon, 43, 461-463.

Reimer, P.J., Bard, E., Bayliss, A., Beck, J.W., Blackwell, P.G., Ramsey, C.B., Buck, C.E., Cheng, H., Edwards, R.L., Friedrich, M., Grootes, P.M., Guilderson, T.P., Haflidason, H., Hajdas, I., Hatte, C., Heaton, T.J., Hoffmann, D.L., Hogg, A.G., Hughen, K.A., Kaiser, K.F., Kromer, B., Manning, S.W., Niu, M., Reimer, R.W., Richards, D.A., Scott, E.M., Southon, J.R., Staff, R.A., Turney, C.S.M., van der Plicht, J., 2013. IntCal13 and Marine13 radiocarbon age calibration curves 0 to 50,000 years BP. Radiocarbon, 55, 1869-1887.

Riggs, S.R., Cleary, W.J., Snyder, S.W., 1995. Influence of inherited geologic framework on barrier shoreface morphology and dynamics. Marine Geology 126, 213-234. http://dx.doi.org/10.1016/0025-3227(95)00079-E.

Rindsberg, A.K., 1992. Holocene ichnology of eastern Mississippi Sound, Alabama: Alabama Geological Survey Circular 167, 1-75.

Roberts, H.H., Fillon, R., Kohl, B., Robalin, J., Sydow, J., 2004. Depositional architecture of the Lagniappe Delta: sediment characteristics, timing of depositional events, and temporal relationship with adjacent shelf-edge deltas, in 
Anderson, J.B., and Fillon, R.H., (Eds.), Late Quaternary Stratigraphic Evolution of the Northern Gulf of Mexico: SEPM, Special Publication 79, 143-188.

Rodriguez, A.B., Anderson, J.B., Simms, A.R., 2008. Response of Mobile Bay and eastern Mississippi Sound, Alabama to changes in sediment accommodation and accumulation. in Anderson, J.B., and Rodriguez, A.B., (Eds.), Response of Upper Gulf Coast Estuaries to Holocene Climate Change and Sea-Level Rise: Geological Society of America Special Paper 443, 13-29.

Rodriguez, A.B., Anderson, J.B., Siringan, F.P., Tavani, M., 2004. Holocene Evolution of the East Texas Coast and Inner Shelf: Along-Strike Variability in Coastal Retreat Rates. Journal of Sedimentary Research. 74, 405-421.

Rodriguez, A.B., Fassell, M.L., Anderson, J.B., 2001. Variations in shoreface progradation and ravinement along the Texas coast, Gulf of Mexico. Sedimentology 48, 837-853.

Rodriguez, A.B., Simms, A.R., Anderson, J.B., 2010. Bay-head deltas across the northern Gulf of Mexico back step in response to the 8.2 ka cooling event. Quaternary Science Reviews 29,3983-3993.

Rodriguez, A.B., Meyer, C.T., 2006. Sea-Level Variation during the Holocene deduced from the morphologic and stratigraphic evolution of Morgan Peninsula, Alabama, USA. Journal of Sedimentary Research 76, 257-269.

Rodriguez, A.B., Yu, W., Theuerkauf, E.J., 2018. Abrupt Increase in Washover Deposition Along a Transgressive Barrier Island During the Late Nineteenth Century Acceleration in Sea Level Rise. In L.J. Moore, A.B., Murray (Eds.), Barrier Dynamics and Response to Changing Climate. Spring, New York doi.org/10.1007/978-3-319-68086-6_4

Rucker, J.B., Snowden, J.O., 1990. Barrier island evolution and reworking by inlet migration along the Mississippi-Alabama Gulf Coast. Transactions of the Gulf Coast Association of Geological Societies 40, 745-753.

Sanford, J.M., Harrison, A.S, Wiese, D.S., Flocks, J.G, 2016a, Archive of Digitized Analog Boomer Seismic Reflection Data Collected from Lake Ponchartrain, Louisiana to Mobile Bay, Alabama, During Cruises Onboard the R/V ERDA-1, June and August, 1992: U.S. Geological Survey Data Series 370.

Sanford, J.M., Harrison, A. S., Wiese, D.S., Flocks, J.G., 2016b, Archive of Digitized Analog Boomer Seismic Reflection Data Collected from the MississippiAlabama-Florida shelf During Cruises Onboard the R/V Kit Jones, June 1990 and July 1991: U.S. Geological Survey Data Series 429.

Sanford, J.M., Harrison, A. S., Wiese, D.S., Flocks, J.G., 2016c, Archive of Digitized Analog Boomer and Minisparker Seismic Reflection Data Collected from the Alabama-Mississippi-Louisiana Shelf During Cruises Onboard the R/V Carancahua and R/V Gyre, April and July, 1981: U.S. Geological Survey Data Series 428.

Schwab, W.C., Baldwin, W.E., Hapke, C.J., Lentz, E.E., Gayes, P.T., Denny, J.F., List, J.H., Warner, J.C., 2013. Geologic evidence for onshore sediment transport from the inner-continental shelf: Fire Island, New York. Journal of Coastal Research 29 (3), 536-544. 
Shackleton, N.J., 1987. Oxygen isotopes, ice volume and sea level. Quaternary Science Reviews 6, 183-190.

Shackleton, N.J., 2000. The 100,000-year Ice-Age cycle identified and found to lag temperature, carbon dioxide, and orbital eccentricity. Science 289, 1897-1902.

Simms, A.R., Anderson, J.B., Rodriguez, A.B., Taviani, M., 2008. Mechanisms controlling environmental change within an estuary: Corpus Christi Bay, Texas, USA. In: Anderson, J.B., Rodriguez, A.B. (Eds.), Response of Upper Gulf Coast Estuaries to Holocene Climate Change and Sea-Level Rise. Geological Society of America, Special Paper 443, 121-146.

Simms, A.R., Anderson, J.B., Blum, M., 2006. Barrier-island aggradation via inlet migration: Mustang Island, Texas. Sedimentary Geology. 187, 105-125.

Siringan, F.P., Anderson, J.B., 1993. Seismic facies, architecture, and evolution of the Bolivar Roads tidal inlet/delta complex, East Texas Gulf Coast. Journal of Sedimentary Petrology 63, 794-808.

Snedden, J., Dalrymple, R., 1999. Modern shelf sand ridges: From historical perspective to a unified hydrodynamic and evolutionary model in: Bergman, K., Snedden, J. (Eds.), Isolated Shallow Marine Sand Bodies: Sequence Stratigraphic Analysis and Sedimentologic Interpretation, 64. SEPM (Society for Sedimentary Geologists) Special Publication No, Tulsa, Oklahoma, 13-28.

Stone, G.W., Stapor Jr., F.W., May, J.P., Morgan, J.P., 1992. Multiple sediment sources and a cellular, non-integrated, longshore drift system: Northwest Florida and southeast Alabama Coast, USA. Marine Geology 105, 141-154.

Swift, D. J.P., 1975 Barrier Island genesis: Evidence from the central Atlantic Shelf, eastern USA: Sedimentary Geology 14, 1-43.

Swift, D.J.P, McKinney, T.F., Stahl, L. 1984. Recognition of transgressive and posttransgressive sand ridges on the New Jersey continental shelf-discussion, in Tillman, R.W. and Siemers, C.T. (Eds.), Siliciclastic Shelf Sediments: Tulsa, SEPM (Society for Sedimentary Geology) Special Publication 34, 25-36.

Timmons, E.A., Rodriguez, A.B., Mattheus, C.R., DeWitt, R., 2010. Transition of a regressive to a transgressive barrier island due to back-barrier erosion, increased storminess, and low sediment supply: Bogue Banks, North Carolina, USA. Marine Geology 278, 100-114.

Thomas, M.A., Anderson, J.B., 1994. Sea-level controls on the facies architecture of the Trinity/Sabine Incised-Valley System, Texas Continental Shelf. In: Dalrymple, R., Boyd, R., Zaitlin, B.A. (Eds.), Incised Valley Systems: Origin and Sedimentary Sequences. Society for Sedimentary Research Special Publication 51, 63-82.

Törnqvist, T.E., Rosenheim, B.R., Hu P., Fernandez, A.B., 2015. Radiocarbon dating and calibration. In: Shennan, I., Long, A.J., and Horton, B.P. (Eds.), Handbook of Sea-Level Research. John Wiley \& Sons, 349-360.

Törnqvist, T.E., Bick, S.J., González, J.L., van der Borg, K., de Jong, A.F.M., 2004a. Tracking the sea-level signature of the 8.2 ka cooling event: New constraints from the Mississippi Delta: Geophysical Research Letters 31, doi:10.1029/2004GL021429. 
Törnqvist, T.E., González, J.L., Newsom, L.A., van der Borg, K., de Jong, A.F.M., Kurnik, C.W., 2004b, Deciphering Holocene sea-level history on the U.S. Gulf Coast: A high-resolution record from the Mississippi Delta: Geological Society of America Bulletin 116, 1026-1039 doi:10.1130/B2525478.1.

Törnqvist, T.E., Bick, S.J., van der Borg, K., de Jong, A.F.M., 2006, How stable is the Mississippi Delta? Geology 34, 697-700 doi:10.1130/G22624.1.

Trowbridge, J.H., 1995. A mechanism for the formation and maintenance of the shore oblique sand ridges on storm-dominated shelves. Journal of Geophysical Research 100, 16,071-16,086.

Twichell, D.C., Pendleton, E., Baldwin, W., Foster, D., Flocks, J., Kelso, K., DeWitt, N., Pfeiffer, W., Forde, A., Krick, J., Baehr, J., 2011. The shallow stratigraphy and sand resources offshore of the Mississippi barrier islands, U.S. Geological Survey Open-file Report 2011-1173, 1-61.

Twichell, D.C.; Flocks, J.G.; Pendleton, E.A., Baldwin, W.E., 2013. Geologic controls on regional and local erosion rates of three northern Gulf of Mexico barrier island systems. In: Brock, J.C.; Barras, J.A., and Williams, S.J. (Eds.), Understanding and Predicting Change in the Coastal Ecosystems of the Northern Gulf of Mexico, Journal of Coastal Research, Special Issue No. 63, 32-45.

Upshaw, C.F., Creath, C.W., Brooks, F.L., 1966. Sediments and microfauna off the coasts of Mississippi and adjacent states: Mississippi Geological Survey Bulleting 106, 1-127.

USACE, 2014. Mississippi Coastal Improvements Program (MsCIP), Appendix A (Offshore Sand Borrow Investigation, 2010-2014, Geotechnical Report) of Supplemental Environmental Impact Statement for Barrier Islands Restoration Plan. Mobile District. Prepared by CH2M Hill, Inc. July, 2014.

Wallace, D.J., Anderson, J.B., 2013. Unprecedented erosion of the upper Texas Coast: Response to accelerated sea-level rise and hurricane impacts. Geological Society of America Bulletin, 125 (5-6), 728-740. https://doi.org/10.1130/B30725.1

Wallace, D.J., Anderson, J.B., Fernández, R., 2010. Transgressive ravinement versus depth of closure: a geological perspective from the upper Texas Coast. J. Coast. Res. 26, 1057-1067.

Wellner, J.L., Sarzalejo, S., Lagoe, M. Anderson, J.B., 2004. Late Quaternary Stratigraphic Evolution of the West Louisiana/East Texas Continental Shelf, in Anderson, J.B., and Fillon, R.H., eds., Late Quaternary Stratigraphic Evolution of the Northern Gulf of Mexico: SEPM, Special Publication 79, 237-271.

Weight, R.W.R., Anderson, J.B., Fernandez, R., 2011. Rapid mud accumulation on the central Texas shelf linked to climate change and sea-level rise. Journal of Sedimentary Research 81, 743-764.

Zaitlin, B.A., Dalrymple, R.W., Boyd, R., 1994. The stratigraphic organization of incised-valley systems associated with relative sea-level change. In: Dalrymple, R.W., Boyd, R., Zaitlin, B.A. (Eds.), Incised-Valley Systems: Origin and Sedimentary Sequences, Spec. Pub. SEPM 51, 45-60. 
Zaremba, N. Mallinson, D., Leorri, E., Culver, S., Riggs, S., Mulligan, R., Horsman, E., Mitra, S., 2016. Controls on the Stratigraphic framework and paleoenvironmental change within a Holocene estuarine system: Pamlico Sound, North Carolina, USA. Marine Geology 379, 109-123. 